\title{
ON REPRESENTATIONS OF 2-BRIDGE KNOT GROUPS IN QUATERNION ALGEBRAS
}

\author{
HUGH M. HILDEN, MARÍA TERESA LOZANO*, \\ AND JOSÉ MARÍA MONTESINOS-AMILIBIA**
}

\begin{abstract}
Representations of two bridge knot groups in the isometry group of some complete Riemannian 3-manifolds as $E^{3}$ (Euclidean 3-space), $H^{3}$ (hyperbolic 3-space) and $E^{2,1}$ (Minkowski 3-space), using quaternion algebra theory, are studied. We study the different representations of a 2-generator group in which the generators are send to conjugate elements, by analyzing the points of an algebraic variety, that we call the variety of affine c-representations of $G$. Each point in this variety correspond to a representation in the unit group of a quaternion algebra and their affine deformations.
\end{abstract}

\section{INTRODUCTION}

The representations of knot groups in special groups have been used to define invariants of knots, as the Alexander polynomial ([6]), A-polynomial ([3]), peripheral polynomials ([10]), etc. Moreover the representations of a knot group $G(K)$ in the group of isometries of a geometric manifold contain the holonomies of the orbifold and cone-manifold structures in $S^{3}$ (or in a manifold obtained by Dehn-surgery in $K$ ) with the knot $K$ as singular locus. We are interested in representations of knot-groups in the isometry group of some complete Riemannian 3-manifolds. In this paper we focus our attention on representations of 2-generator groups mapping the generators to conjugate elements, because this case has immediate application to two-bridge knot groups and to a special case of representations of two bridge link groups.

It turns out that the quaternion algebra theory is useful to study in a comprehensive way the groups of isometries of some 3-dimensional Riemannian 3manifolds as $E^{3}$ (Euclidean 3-space), $H^{3}$ (hyperbolic 3-space) and $E^{2,1}$ (Minkowski 3 -space). The group of orientation preserving isometries of hyperbolic 3 -space $H^{3}$ is $\operatorname{PSL}(2, \mathbb{C}) \cong S L(2, \mathbb{C}) /\{ \pm I\}$, where $S L(2, \mathbb{C})$ is the group of unit quaternions in the quaternion algebra $M(2, \mathbb{C})=\left(\frac{-1,1}{\mathbb{C}}\right)$. We explain in this paper how the group of orientation preserving isometries of the Euclidean 3-space $E^{3}$ is associated with the Hamilton quaternions $H=\left(\frac{-1,-1}{\mathbb{R}}\right)$, and the group of isometries preserving orientation of the Minkowski 3-space $E^{1,2}$ is related with the quaternion algebra $M(2, R)=\left(\frac{-1,1}{\mathbb{R}}\right)$, in such a way that we can study the different representations of a 2-generator group by analyzing the points of an algebraic variety, that we call the variety of affine c-representations of $G$. The points in this variety correspond to representations in the unit group of a quaternion algebra and their affine deformations. These representations are important in order to relate knot groups with affine crystallographic groups and Lorentz structures. (For the definition and relevance of these concepts see, for instance, [7, [2], 8$]$.)

Date: May, 2008.

2000 Mathematics Subject Classification. 57M50, 57M25, 57M60.

Key words and phrases. quaternion algebra, representation, knot group.

*This research was supported by grant MTM2007-67908-C02-01.

**This research was supported by grant MTM2006-00825. 
In Section 2 we review some concepts and results on Quaternion algebra theory. (See [15] as general reference). In Section 3] we characterize a pair of unit quaternions up to conjugation in different quaternion algebras. In Section 4, Theorem 3 describes a new algorithm to obtain the ideal defining the algebraic variety $V\left(\mathcal{I}_{G}^{c}\right)$ of c-representations of $G$ in $S L(2, \mathbb{C})$ giving explicitly the c-representation associated to each point in the variety $V\left(\mathcal{I}_{G}^{c}\right)$ and it corresponding minimal quaternion algebra. Theorem 4 gives the complete classification of c-representations of $G$ in $S^{3}$ and $S L(2, \mathbb{R})$. We apply Theorems 3 and 4 to the group of the Trefoil knot (a no hyperbolic knot) and to the group of the Figure Eight knot (a hyperbolic knot). Finally in Section [5] we characterize a pair of conjugate affine isometries and we obtain the ideal defining the variety $V_{a}\left(\mathcal{I}_{a G}^{c}\right)$ of affine c-representations of $G$. As an example we obtain the ideal $\mathcal{I}_{a G}^{c}$ for the Trefoil knot and the Figure Eight knot.

\section{QUATERNION ALGEBRAS}

\subsection{Definitions.}

Definition 1. Let $k$ be a number field and let be $\mu, \nu \in k^{*}$, where $k^{*}$ is the group of invertible elements in $k$. The quaternion algebra $H=\left(\frac{\mu, \nu}{k}\right)$ is the $k$-algebra on two generators $i, j$ with the defining relations:

$$
i^{2}=\mu, \quad j^{2}=\nu \quad \text { and } \quad i j=-j i .
$$

An easy consequence of the definition is that $H$ is a four dimensional vector space over $k$, with basis $\{1, i, j, i j\}$. The square of $i j$ is also an invertible element in $k$ :

$$
(i j)(i j)=(i j)(-j i)=-i j j i=-\mu \nu \in k^{*} .
$$

Proposition 1. For any $k$, the quaternion algebra $\left(\frac{-1,1}{k}\right)$ is isomorphic to the algebra $M(2, k)$ of $2 \times 2$ matrices over $k$ :

$$
\begin{array}{ccc}
\left(\frac{-1,1}{k}\right)=\langle 1, i, j, i j\rangle & \rightarrow & M(2, k) \\
i & \rightarrow & I=\left(\begin{array}{cc}
0 & 1 \\
-1 & 0
\end{array}\right) \\
j & \rightarrow & J=\left(\begin{array}{cc}
0 & 1 \\
1 & 0
\end{array}\right)
\end{array}
$$

Proposition 2. For the complex field $\mathbb{C}$, there is only one quaternion algebra, up to isomorphism, the algebra $M(2, \mathbb{C})$. In fact, given two nonzero complex numbers, $\mu, \nu$, there exists $x, y \in \mathbb{C}$, such that $\mu=x^{2}$ and $\nu=y^{2}$. Then the map

$$
\begin{array}{ccc}
\left(\frac{\mu, \nu}{\mathbb{C}}\right)=\langle 1, i, j, i j\rangle & \longrightarrow & M(2, \mathbb{C})=\left(\frac{-1,1}{\mathbb{C}}\right)=\langle 1, I, J, I J\rangle \\
i & \rightarrow & y J \\
j & \rightarrow &
\end{array}
$$

is an algebra isomorphism.

Easy consequences of the above result are the following:

Proposition 3. Any quaternion algebra $H=\left(\frac{\mu, \nu}{k}\right), k \subset \mathbb{C}$, is a subalgebra of the algebra $M(2, \mathbb{C})=\left(\frac{-1,1}{\mathbb{C}}\right)$.

Proposition 4. For the real field $\mathbb{R}$, there are only two quaternion algebras, up to isomorphism, the algebra $M(2, \mathbb{R})=\left(\frac{-1,1}{\mathbb{R}}\right)$ and the Hamilton quaternions $\mathbb{H}=$ $\left(\frac{-1,-1}{\mathbb{R}}\right)$.

Given a quaternion $A=\alpha+\beta i+\gamma j+\delta i j, A \in H=\left(\frac{\mu, \nu}{k}\right)=\langle 1, i, j, i j\rangle$, we use the notation $A^{+}=\alpha$, and $A^{-}=\beta i+\gamma j+\delta i j$. Then, $A=A^{+}+A^{-}$. Compare [1]. 
Definition 2. The conjugate of $A$, is by definition, the quaternion

$$
\bar{A}:=A^{+}-A^{-}=\alpha-\beta i-\gamma j-\delta i j .
$$

The norm of $A$ is $N(A):=A \bar{A}=\bar{A} A$. Observe that

$N(A)=(\alpha+\beta i+\gamma j+\delta i j)(\alpha-\beta i-\gamma j-\delta i j)=\alpha^{2}-\beta^{2} \mu-\gamma^{2} \nu+\delta^{2} \mu \nu \in k$.

The trace of $A$ is $T(A)=A+\bar{A}$. Observe that

$$
T(A)=2 A^{+}=2 \alpha \in k .
$$

The map

$$
\begin{aligned}
& \langle, \quad\rangle: H \times H \rightarrow \quad k \\
& (A, B) \quad \rightarrow \quad \frac{1}{2}(A \bar{B}+B \bar{A})=\frac{1}{2} T(A \bar{B})=(A \bar{B})^{+}
\end{aligned}
$$

is a symmetric bilinear form on $H$. The associated quadratic form

$$
\begin{array}{ccc}
H & \longrightarrow & k \\
A & \rightarrow & \frac{1}{2} T(A \bar{A})=\frac{1}{2} 2 N(A)=N(A)
\end{array}
$$

is the norm form $N$ on $H$. We denote by $(H, N)$ the quadratic structure in $H$. Observe that the associated matrix to the norm form on $H$ in the basis $\{1, \quad i, \quad j, \quad i j\}$, is the matrix $\left(\begin{array}{cccc}1 & 0 & 0 & 0 \\ 0 & -\mu & 0 & 0 \\ 0 & 0 & -\nu & 0 \\ 0 & 0 & 0 & \mu \nu\end{array}\right)$.

2.2. Examples. We are mostly interested in the following three examples: $M(2, \mathbb{C})$, $\mathbb{H}$ and $M(2, \mathbb{R})$.

2.2.1. $M(2, \mathbb{C})$. The quaternion algebra $M(2, \mathbb{C})=\left(\frac{-1,1}{\mathbb{C}}\right)$ (See Proposition 1). The trace is the usual trace of the matrix, and the norm is the determinant of the matrix. Proposition 2 shows that it is possible to change the basis to obtain any possible pair $(\mu, \nu)$ of complex numbers defining the structure of quaternion algebra $\left(\frac{\mu, \nu}{\mathbb{C}}\right)$. In particular

$$
M(2, \mathbb{C})=\left(\frac{-1,1}{\mathbb{C}}\right)=\left(\frac{1,1}{\mathbb{C}}\right)=\left(\frac{-1,-1}{\mathbb{C}}\right)
$$

Next we analyze, using Proposition 2, the basis and the corresponding matrix associated to the norm form for this three presentations.

(1) $M(2, \mathbb{C})=\left(\frac{-1,1}{\mathbb{C}}\right)$. As in Proposition 1, we consider the basis $\{$ Ident, $I, J, I J\}$, where

$$
I=\left(\begin{array}{cc}
0 & 1 \\
-1 & 0
\end{array}\right), \quad J=\left(\begin{array}{ll}
0 & 1 \\
1 & 0
\end{array}\right), \quad I J=\left(\begin{array}{cc}
1 & 0 \\
0 & -1
\end{array}\right)
$$

For $A=\alpha+\beta I+\gamma J+\delta I J=\left(\begin{array}{cc}\alpha+\delta & \beta+\gamma \\ -\beta+\gamma & \alpha-\delta\end{array}\right) \in M(2, \mathbb{C}), \alpha, \beta, \gamma, \delta \in \mathbb{C}$, $N(A)=\alpha^{2}+\beta^{2}-\gamma^{2}-\delta^{2}$. Therefore the matrix associated to the quadratic form in the basis $\{$ Ident $, I, J, I J\}$, is the matrix $\left(\begin{array}{cccc}1 & 0 & 0 & 0 \\ 0 & 1 & 0 & 0 \\ 0 & 0 & -1 & 0 \\ 0 & 0 & 0 & -1\end{array}\right)$.

(2) $M(2, \mathbb{C})=\left(\frac{1,1}{\mathbb{C}}\right)$. Using Proposition 2, $x=1, y=1$, we consider the basis $\left\{\right.$ Ident, $\left.I_{0}, J_{0}, I_{0} J_{0}\right\}$, where

$$
\begin{gathered}
I_{0}=\sqrt{-1} I=\left(\begin{array}{cc}
0 & \sqrt{-1} \\
-\sqrt{-1} & 0
\end{array}\right), \quad J_{0}=J=\left(\begin{array}{ll}
0 & 1 \\
1 & 0
\end{array}\right), \\
I_{0} J_{0}=\left(\begin{array}{cc}
\sqrt{-1} & 0 \\
0 & -\sqrt{-1}
\end{array}\right)
\end{gathered}
$$


For $A=\alpha+\beta I_{0}+\gamma J_{0}+\delta I_{0} J_{0}=\left(\begin{array}{ll}\alpha+\delta \sqrt{-1} & \gamma+\beta \sqrt{-1} \\ \gamma-\beta \sqrt{-1} & \alpha-\delta \sqrt{-1}\end{array}\right) \in M(2, \mathbb{C})$, where $\alpha, \beta, \gamma, \delta \in \mathbb{C}, N(A)=\alpha^{2}-\beta^{2}-\gamma^{2}+\delta^{2}$. Therefore the matrix associated to the quadratic form in the basis $\left\{\right.$ Ident, $\left.I_{0}, J_{0}, I_{0} J_{0}\right\}$, is the $\operatorname{matrix}\left(\begin{array}{cccc}1 & 0 & 0 & 0 \\ 0 & -1 & 0 & 0 \\ 0 & 0 & -1 & 0 \\ 0 & 0 & 0 & 1\end{array}\right)$

(3) $M(2, \mathbb{C})=\left(\frac{-1,-1}{\mathbb{C}}\right)$. Using Proposition $2, x=\sqrt{-1}, y=\sqrt{-1}$, we consider the basis $\left\{\right.$ Ident, $\left.I_{1}, J_{1}, I_{1} J_{1}\right\}$, where

$$
\begin{gathered}
I_{1}=\sqrt{-1} \sqrt{-1} I=-I=\left(\begin{array}{cc}
0 & -1 \\
1 & 0
\end{array}\right), \quad J_{1}=\sqrt{-1} J=\left(\begin{array}{cc}
0 & \sqrt{-1} \\
\sqrt{-1} & 0
\end{array}\right), \\
I_{1} J_{1}=\left(\begin{array}{cc}
-\sqrt{-1} & 0 \\
0 & \sqrt{-1}
\end{array}\right)
\end{gathered}
$$

For $A=\alpha+\beta I_{1}+\gamma J_{1}+\delta I_{1} J_{1}=\left(\begin{array}{cc}\alpha-\delta \sqrt{-1} & -\beta+\gamma \sqrt{-1} \\ \beta+\gamma \sqrt{-1} & \alpha+\delta \sqrt{-1}\end{array}\right) \in M(2, \mathbb{C})$, where $\alpha, \beta, \gamma, \delta \in \mathbb{C}, N(A)=\alpha^{2}+\beta^{2}+\gamma^{2}+\delta^{2}$. Therefore the matrix associated to the quadratic form in the basis $\left\{\right.$ Ident, $\left.I_{1}, J_{1}, I_{1} J_{1}\right\}$, is the $\operatorname{matrix}\left(\begin{array}{cccc}1 & 0 & 0 & 0 \\ 0 & 1 & 0 & 0 \\ 0 & 0 & 1 & 0 \\ 0 & 0 & 0 & 1\end{array}\right)$

2.2.2. $\mathbb{H}$. The quaternion algebra $\mathbb{H}=\left(\frac{-1,-1}{\mathbb{R}}\right)$ is the algebra of Hamilton quaternions. It is isomorphic to an $\mathbb{R}$-subalgebra of the $\mathbb{C}$-algebra $M(2, \mathbb{C})=\left(\frac{-1,-1}{\mathbb{C}}\right)$.

$$
\begin{array}{clc}
\Phi: \mathbb{H}=\left(\frac{-1,-1}{\mathbb{R}}\right)=\langle 1, i, j, i j\rangle & \longrightarrow & M(2, \mathbb{C}) \\
i & \rightarrow & I_{1}=\left(\begin{array}{cc}
0 & -1 \\
1 & 0
\end{array}\right) \\
j & \rightarrow & J_{1}=\left(\begin{array}{cc}
0 & \sqrt{-1} \\
\sqrt{-1} & 0
\end{array}\right)
\end{array}
$$

For $A=\alpha+\beta i+\gamma j+\delta i j \in \mathbb{H}, N(A)=\alpha^{2}+\beta^{2}+\gamma^{2}+\delta^{2}$, where $\alpha, \beta, \gamma, \delta \in \mathbb{R}$. Therefore the matrix associated to the quadratic form in the basis $\{1, i, j, i j\}$, is the Identity matrix $\left(\begin{array}{cccc}1 & 0 & 0 & 0 \\ 0 & 1 & 0 & 0 \\ 0 & 0 & 1 & 0 \\ 0 & 0 & 0 & 1\end{array}\right)$. Note that $\Phi(A)=\Phi(\alpha+\beta i+\gamma j+\delta i j)=$ $\left(\begin{array}{cc}\alpha-\delta \sqrt{-1} & -\beta+\gamma \sqrt{-1} \\ \beta+\gamma \sqrt{-1} & \alpha+\delta \sqrt{-1}\end{array}\right) \in M(2, \mathbb{C})$.

2.2.3. $M(2, \mathbb{R})$. The quaternion algebra $M(2, \mathbb{R})=\left(\frac{-1,1}{\mathbb{R}}\right)$. See Proposition 1] It is isomorphic to an $\mathbb{R}$-subalgebra of the $\mathbb{C}$-algebra $M(2, \mathbb{C})=\left(\frac{-1,1}{\mathbb{C}}\right)$.

$$
\begin{array}{cl}
\Psi: M(2, \mathbb{R})=\left(\frac{-1,1}{\mathbb{R}}\right)=\langle 1, I, J, I J\rangle & \rightarrow \\
I & \rightarrow \quad I=\left(\begin{array}{cc}
0 & 1 \\
-1 & 0
\end{array}\right) \\
J & \rightarrow \quad J=\left(\begin{array}{ll}
0 & 1 \\
1 & 0
\end{array}\right)
\end{array}
$$

For $A=\alpha+\beta I+\gamma J+\delta I J \in M(2, \mathbb{R}), N(A)=\alpha^{2}+\beta^{2}-\gamma^{2}-\delta^{2}$, where $\alpha, \beta, \gamma, \delta \in \mathbb{R}$. Therefore the matrix associated to the quadratic form in the basis 
$\{1, I, J, I J\}$, is the matrix $\left(\begin{array}{cccc}1 & 0 & 0 & 0 \\ 0 & 1 & 0 & 0 \\ 0 & 0 & -1 & 0 \\ 0 & 0 & 0 & -1\end{array}\right)$. Note that $\Psi(A)=\Psi(\alpha+\beta I+$ $\gamma J+\delta I J)=\left(\begin{array}{cc}\alpha+\delta & \beta+\gamma \\ -\beta+\gamma & \alpha-\delta\end{array}\right) \in M(2, \mathbb{C})$

2.3. Pure and unit quaternions. There are two important subsets in the quaternion algebra $H=\left(\frac{\mu, \nu}{k}\right)$. The pure quaternions $H_{0}$ (a 3-dimensional vector space), and the unit quaternions $U_{1}$ (a multiplicative group).

The pure quaternions $H_{0}=\left\{A \in H: A^{+}=0\right\}$ form a three dimensional vector space over $k$ generated by $\{i, j, i j\}$. The symmetric bilinear form $\langle, \quad\rangle$ restricts to $H_{0}$ defining the quadratic space $\left(H_{0}, N\right)$. Because $\overline{A^{-}}=-A^{-}$, we have that

$$
\left\langle A^{-}, B^{-}\right\rangle=\frac{1}{2}\left(A^{-} \overline{B^{-}}+B^{-} \overline{A^{-}}\right)=\frac{1}{2}\left(-A^{-} B^{-}-B^{-} A^{-}\right)=-\left(A^{-} B^{-}\right)^{+}
$$

Therefore two elements $A^{-}, B^{-} \in H_{0}$ are orthogonal for the symmetric bilinear form $\langle\rangle,,\left(\left\langle A^{-}, B^{-}\right\rangle=0\right)$ if and only if $A^{-}, B^{-}$anticommute. The elements $i, j, i j$ anticommute. Then $\{i, j, i j\}$ is a orthogonal basis in $H_{0}$. In this basis the associated matrix to the restricted symmetric bilinear form $\langle,\rangle_{\mid H_{0}}$ is $\left(\begin{array}{ccc}-\mu & 0 & 0 \\ 0 & -\nu & 0 \\ 0 & 0 & \mu \nu\end{array}\right)$

Denote by $O\left(H_{0}, N\right)$ the orthogonal group for the quadratic space $\left(H_{0}, N\right)$ :

$$
O\left(H_{0}, N\right)=\left\{\varphi: H_{0} \cong H_{0}: N\left(\varphi\left(A^{-}\right)\right)=N\left(A^{-}\right), A^{-} \in H_{0}\right\}
$$

The isomorphism $\left(H_{0}, N\right) \cong\left(k^{3},\left(\begin{array}{ccc}-\mu & 0 & 0 \\ 0 & -\nu & 0 \\ 0 & 0 & \mu \nu\end{array}\right)\right)$, defined by the basis $\{i, j, i j\}$, induced the isomorphism

$$
O\left(H_{0}, N\right) \cong\left\{M \in G L(3, k): M^{t}\left(\begin{array}{ccc}
-\mu & 0 & 0 \\
0 & -\nu & 0 \\
0 & 0 & \mu \nu
\end{array}\right) M=\left(\begin{array}{ccc}
-\mu & 0 & 0 \\
0 & -\nu & 0 \\
0 & 0 & \mu \nu
\end{array}\right)\right\}
$$

The special orthogonal group is defined by

$$
S O\left(H_{0}, N\right)=\left\{M \in O\left(H_{0}, N\right): \operatorname{det}(M)=1\right\} .
$$

Consider the group $U$ of invertible elements in $H$. There exits a short exact sequence of groups

$$
1 \longrightarrow k^{*} \longrightarrow U \stackrel{c}{\longrightarrow} S O\left(H_{0}, N\right) \longrightarrow 1
$$

such that $c(A)$ acts in $H_{0}$ by conjugation : $c(A)\left(B^{-}\right)=A B^{-} A^{-1}$. See [15, p. 63].

The unit quaternions $U_{1}$ are the elements in the group $U$ with norm 1 .

We will also consider the group $U_{ \pm 1}$ of quaternions with norm \pm 1 . It is a subgroup of $U$ and either it coincides with $U_{1}$ or has $U_{1}$ as a subgroup of index 2 .

$$
U_{1} \triangleleft U_{ \pm 1}<U
$$

2.3.1. The matrices and action of $c(A)$ as linear map in $H_{0}$. Let $H_{0}$ be the vector space of pure quaternions in a quaternion algebra $H=\left(\frac{\mu, \nu}{k}\right)$. Consider a coordinate system $\{X, Y, Z\}$ in $H_{0}$ in the basis $\{-i j, j, i\}$, such that a pure quaternion is represented by $Z i+Y j-X i j$. For a unit quaternion $A=\alpha+\beta i+\gamma j+\delta i j$, 
$c(A)$ acts in $H_{0}$ as a linear map, therefore there exists an associated $3 \times 3$ matrix $m(\mu, \nu ; \alpha, \beta, \gamma, \delta)$ such that

$$
\left(\begin{array}{l}
X^{\prime} \\
Y^{\prime} \\
Z^{\prime}
\end{array}\right)=m(\mu, \nu ; \alpha, \beta, \gamma, \delta)\left(\begin{array}{l}
X \\
Y \\
Z
\end{array}\right)
$$

where $\left(Z^{\prime} i+Y^{\prime} j-X^{\prime} i j\right)=c(A)((Z i+Y j-X i j))=(\alpha+\beta i+\gamma j+\delta i j)(Z i+Y j-$ $X i j)(\alpha-\beta i-\gamma j-\delta i j)$. Then a straightforward computation yields the following explicit matrix

(2.3) $m(\mu, \nu ; \alpha, \beta, \gamma, \delta)=$

$$
=\left(\begin{array}{ccc}
\alpha^{2}+\mu \beta^{2}+\nu \gamma^{2}+\mu \nu \delta^{2} & -2 \alpha \beta+2 \nu \gamma \delta & 2 \alpha \gamma+2 \mu \beta \delta \\
-2 \mu \alpha \beta-2 \mu \nu \gamma \delta & \alpha^{2}+\mu \beta^{2}-\nu \gamma^{2}-\mu \nu \delta^{2} & -2 \mu \beta \gamma-2 \mu \alpha \delta \\
2 \nu \alpha \gamma-2 \mu \nu \beta \delta & -2 \nu \beta \gamma+2 \nu \alpha \delta & \alpha^{2}-\mu \beta^{2}+\nu \gamma^{2}-\mu \nu \delta^{2}
\end{array}\right)
$$

Remark 1. We use the coordinate system $\{X, Y, Z\}$ in $H_{0}$ in the basis $\{-i j, j, i\}$, in order to have the usual pictures in the particular case of the Minkowski space, as we will see in Example 3 .

Example 1. $M(2, \mathbb{C})$. In the case $H=M(2, \mathbb{C})=\left(\frac{-1,-1}{\mathbb{C}}\right)$ there exits a short exact sequence of groups

$$
1 \longrightarrow\{ \pm 1\} \longrightarrow U_{1} \stackrel{c}{\longrightarrow} S O\left(H_{0}, N\right) \longrightarrow 1
$$

where $U_{1}=\{A \in U: N(A)=1\}=S L(2, \mathbb{C}) . U_{1} \neq U_{ \pm 1}$. Here $\left(H_{0}, N\right)$ is the 3-dimensional complex space $\mathbb{C}^{3}$, with the symmetric bilinear form defined by the identity matrix in the basis $\left\{\right.$ Ident, $\left.I_{1}, J_{1}, I_{1} J_{1}\right\}$, because $\mu=\nu=-1$, and $S O\left(H_{0}, N\right) \cong S O(3, \mathbb{C})$. The above short exact sequence defines the 2-fold covering

$$
U_{1} \cong S L(2, \mathbb{C}) \stackrel{c}{\longrightarrow} S O(3, \mathbb{C})
$$

and the isomorphism $P S L(2, C) \cong S O(3, \mathbb{C})$.

Example 2. $\mathbb{H}$. In the case $\mathbb{H}=\left(\frac{-1,-1}{\mathbb{R}}\right)$ there exits a short exact sequence of groups

$$
1 \longrightarrow\{ \pm 1\} \longrightarrow U_{1} \stackrel{c}{\longrightarrow} S O\left(H_{0}, N\right) \longrightarrow 1
$$

where $U_{1}=\{A \in U: N(A)=1\}=U_{ \pm 1}$ is the group of unit quaternions. Here $U_{1} \cong S^{3},\left(H_{0}, N\right)$ is the Euclidean 3-dimensional real space $E^{3}$, because $\mu=\nu=$ -1 , and $S O\left(H_{0}, N\right) \cong S O(3, \mathbb{R})$. The above short exact sequence defines the 2-fold covering

$$
U_{1} \cong S^{3} \stackrel{c}{\longrightarrow} S O(3, \mathbb{R})
$$

Observe that the inclusion $\Phi: \mathbb{H}=\left(\frac{-1,-1}{\mathbb{R}}\right) \longrightarrow M(2, \mathbb{C})$, maps the unit quaternions $S^{3}$ isomorphically on $S U(2) \subset S L(2, \mathbb{C})$. Let $A=\alpha+\beta i+\gamma j+\delta i j \in U_{1} \cong S^{3}$, then $1=N(A)=\alpha^{2}+\beta^{2}+\gamma^{2}+\delta^{2}$, and

$$
\Phi(A)=\Phi(\alpha+\beta i+\gamma j+\delta i j)=\left(\begin{array}{cc}
\alpha-\delta \sqrt{-1} & -\beta+\gamma \sqrt{-1} \\
\beta+\gamma \sqrt{-1} & \alpha+\delta \sqrt{-1}
\end{array}\right)=\left(\begin{array}{cc}
z_{1} & -z_{2} \\
\overline{z_{2}} & \overline{z_{1}}
\end{array}\right)
$$

which is an element of $S U(2)=\left\{M \in M(2, \mathbb{C}) ; M^{-1}=\overline{M^{t}}\right\} \subset S L(2, \mathbb{C})$. Reciprocally, every element of $S U(2)$ is of the form $\left(\begin{array}{cc}\frac{z_{1}}{z_{2}} & -z_{2} \\ z_{1}\end{array}\right)$, and therefore is the image by $\Phi$ of an element of $S^{3}$.

Next, we analyze the action of $c(A)$ as a linear map, $A \in U_{1} \cong S^{3}$, in $\left(\mathbb{H}_{0}, N\right) \cong$ $E^{3}$.

$$
c(A): E^{3} \longrightarrow E^{3}
$$


Consider $A=\alpha+\beta i+\gamma j+\delta i j \in U_{1}$, then $1=N(A)=\alpha^{2}+\beta^{2}+\gamma^{2}+\delta^{2}$. Therefore, because $\alpha^{2} \leq 1$, we can assume that $A^{+}=\alpha=\cos \left(\frac{\theta}{2}\right)$, and $N\left(A^{-}\right)=$ $-A^{-} A^{-}=\beta^{2}+\gamma^{2}+\delta^{2}=1-\alpha^{2}=1-\cos ^{2}\left(\frac{\theta}{2}\right)=\sin ^{2}\left(\frac{\theta}{2}\right)$.

Proposition 5. The action of $c(A)$ on $H_{0} \cong E^{3}$ is a right rotation with angle $\theta$ around the oriented axis $A^{-}$. (We assume $0 \leq \theta \leq \pi$ )

Proof. Any element $A=A^{+}+A^{-}=\alpha+A^{-}$in the group $U_{1}$ is conjugate in $U_{1}$ to one with the same trace $2 \alpha$, say $\alpha=\cos \left(\frac{\theta}{2}\right)$ and $\gamma=\delta=0$.

Then, it is enough to consider

$$
A=\cos \left(\frac{\theta}{2}\right)+\sin \left(\frac{\theta}{2}\right) i
$$

The matrix of the action of $c(A)$ as a linear map (see (2.3) $)$ on the basis $\{-i j, j, i\}$ of $E^{3}$ is:

$$
\begin{aligned}
m(-1, & \left.-1 ; \cos \left(\frac{\theta}{2}\right), \sin \left(\frac{\theta}{2}\right), 0,0\right)= \\
= & \left(\begin{array}{ccc}
\cos ^{2}\left(\frac{\theta}{2}\right)-\sin ^{2}\left(\frac{\theta}{2}\right) & -2 \cos \left(\frac{\theta}{2}\right) \sin \left(\frac{\theta}{2}\right) & 0 \\
2 \cos \left(\frac{\theta}{2}\right) \sin \left(\frac{\theta}{2}\right) & \cos ^{2}\left(\frac{\theta}{2}\right)-\sin ^{2}\left(\frac{\theta}{2}\right) & 0 \\
0 & 0 & 1
\end{array}\right)=\left(\begin{array}{ccc}
\cos \theta & -\sin \theta & 0 \\
\sin \theta & \cos \theta & 0 \\
0 & 0 & 1
\end{array}\right)
\end{aligned}
$$

Example 3. $M(2, \mathbb{R})$. In $M(2, \mathbb{R})=\left(\frac{-1,1}{\mathbb{R}}\right)$, The 3-dimensional vector space of pure quaternions with the norm form is isomorphic to the Minkowski space $E^{1,2}$, because the matrix of the quadratic form $\langle, \quad\rangle$ in the basis $\{I, J, I J\}$ is

$$
\left(\begin{array}{ccc}
-\mu & 0 & 0 \\
0 & -\nu & 0 \\
0 & 0 & \mu \nu
\end{array}\right)=\left(\begin{array}{ccc}
1 & 0 & 0 \\
0 & -1 & 0 \\
0 & 0 & -1
\end{array}\right)
$$

Choose a component $\mathcal{N}_{+}$of the complement of 0 in the nullcone or light cone

$$
\mathcal{N}=\left\{q \in E^{1,2}:\langle q, q\rangle=0\right\}
$$

Denote by $S^{+}(1,2)$ the index 2 subgroup of $S O(1,2)$ preserving the component $\mathcal{N}_{+}$, it is also the connected component of the group $S O(1,2)$ containing the identity matrix.

$$
S O^{+}(1,2)=\left\{\begin{array}{c}
M \in S L(3, \mathbb{R}): M^{t}\left(\begin{array}{ccc}
1 & 0 & 0 \\
0 & -1 & 0 \\
0 & 0 & -1
\end{array}\right) M=\left(\begin{array}{ccc}
1 & 0 & 0 \\
0 & -1 & 0 \\
0 & 0 & -1
\end{array}\right), \\
M\left(\begin{array}{l}
1 \\
0 \\
0
\end{array}\right)=\left(\begin{array}{c}
x>0 \\
y \\
z
\end{array}\right)
\end{array}\right\}
$$

In this case there also exist a short exact sequence of groups

$$
1 \longrightarrow\{ \pm 1\} \longrightarrow U_{1} \stackrel{c}{\longrightarrow} S O^{+}(1,2) \longrightarrow 1
$$

Observe that the inclusion $\Psi: M(2, \mathbb{R})=\left(\frac{-1,1}{\mathbb{R}}\right) \longrightarrow M(2, \mathbb{C})$, maps the unit quaternions $U_{1}$ isomorphically onto the subgroup $S L(2, \mathbb{R}) \subset S L(2, \mathbb{C})$. Observe that $U_{1} \neq U_{ \pm 1}$. Let $A=\alpha+\beta I+\gamma J+\delta I J \in U_{1}$, then $1=N(A)=\alpha^{2}+\beta^{2}-\gamma^{2}-\delta^{2}$, and

$$
\Psi(A)=\Psi(\alpha+\beta I+\gamma J+\delta I J)=\left(\begin{array}{cc}
\alpha+\delta & \beta+\gamma \\
-\beta+\gamma & \alpha-\delta
\end{array}\right)
$$


which is an element of $S L(2, \mathbb{R})$ because it is a real matrix with determinant equal to 1 :

$$
\begin{aligned}
\operatorname{Det}\left(\begin{array}{cc}
\alpha+\delta & \beta+\gamma \\
-\beta+\gamma & \alpha-\delta
\end{array}\right) & =(\alpha+\delta)(\alpha-\delta)-(\beta+\gamma)(-\beta+\gamma) \\
& =\alpha^{2}+\beta^{2}-\gamma^{2}-\delta^{2}=N(A)=1
\end{aligned}
$$

Reciprocally, every element $\left(\begin{array}{ll}a & b \\ c & d\end{array}\right) \in S L(2, \mathbb{R})$ is the image by $\Psi$ of the element $A=\alpha+\beta I+\gamma J+\delta I J$ where $\alpha=\frac{a+d}{2}, \beta=\frac{b-c}{2}, \gamma=\frac{b+c}{2}, \delta=\frac{a-d}{2}$, and $A \in U_{1}$ because $\alpha^{2}+\beta^{2}-\gamma^{2}-\delta^{2}=a d-b c=1$. Note that considering a coordinate system $\{X, Y, Z\}$ in $H_{0}$ in the basis $\{-I J, J, I\}$, the unit pure quaternions $U_{1} \cap H_{0}$ constitute the two sheeted hyperboloid: $Z^{2}-Y^{2}-X^{2}=1$. The pure quaternions with norm -1 are the points in the deSitter sphere: $Z^{2}-Y^{2}-X^{2}=-1$. See Figure 11.

To study the element $c(A), A \in U_{1}$ as a linear map in $E^{1,2}$, we consider three cases according to the value of $N\left(A^{-}\right)(>,<,=) 0$. Note that $N(A)=N\left(A^{+}\right)+$ $N\left(A^{-}\right)=\left(A^{+}\right)^{2}+N\left(A^{-}\right)=1$. Then $N\left(A^{-}\right)=1-\left(A^{+}\right)^{2}$.

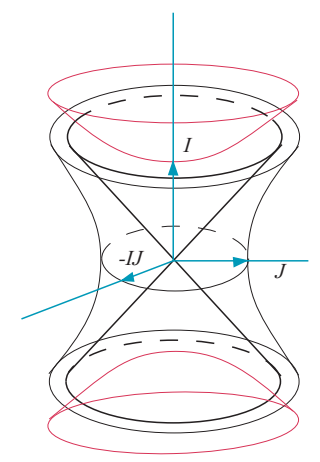

Figure 1. The Minkowski space $E^{1,2}$.

Case $1 N(A)=1, N\left(A^{-}\right)>0$. The vector $A^{-}$is inside the light cone. We say that $A^{-}$is a time-like vector. Then $A=\cos \left(\frac{\theta}{2}\right)+\sin \left(\frac{\theta}{2}\right) P^{-}$where $N\left(P^{-}\right)=1$. Because the kernel of $c$ is $\{ \pm 1\}$, we assume that the $Z$-coordinate of $P^{-}$is positive. Then $P^{-}$is a point in the upper sheet of the two sheeted hyperboloid defined by the unit pure quaternions. Up to conjugation (mapping $P^{-}$to $\left.I\right)$

$$
A=\cos \left(\frac{\theta}{2}\right)+\sin \left(\frac{\theta}{2}\right) I
$$

The matrix of the action of $c(A)$ as a linear map (see (2.3) ) on the basis $\{-I J, J, I\}$ of $E^{1,2}$ is:

$$
\begin{aligned}
& m\left(-1,1 ; \cos \left(\frac{\theta}{2}\right), \sin \left(\frac{\theta}{2}\right), 0,0\right)= \\
& \quad=\left(\begin{array}{ccc}
\cos ^{2}\left(\frac{\theta}{2}\right)-\sin ^{2}\left(\frac{\theta}{2}\right) & -2 \cos \left(\frac{\theta}{2}\right) \sin \left(\frac{\theta}{2}\right) & 0 \\
2 \cos \left(\frac{\theta}{2}\right) \sin \left(\frac{\theta}{2}\right) & \cos ^{2}\left(\frac{\theta}{2}\right)-\sin ^{2}\left(\frac{\theta}{2}\right) & 0 \\
0 & 0 & 1
\end{array}\right)=\left(\begin{array}{ccc}
\cos \theta & -\sin \theta & 0 \\
\sin \theta & \cos \theta & 0 \\
0 & 0 & 1
\end{array}\right)
\end{aligned}
$$

Then the action of $c\left(\cos \left(\frac{\theta}{2}\right)+\sin \left(\frac{\theta}{2}\right) I\right)$ is a (Euclidean) positive rotation around the oriented axis $I$ with angle $\theta$. In the general case, $A=\cos \left(\frac{\theta}{2}\right)+$ $\sin \left(\frac{\theta}{2}\right) P^{-}, c(A)$ acts as a (hyperbolic) rotation around the oriented axis $P^{-}$ with angle $\theta$, preserving its orthogonal plane $\left(P^{-}\right)^{\perp}=\left\{B^{-}:\left\langle P^{-}, B^{-}\right\rangle=0\right\}$. 
To understand this rotation consider in $R P^{2}$ the conic defined by the nullcone as the boundary of the hyperbolic plane $H^{2}$. See Figure 2

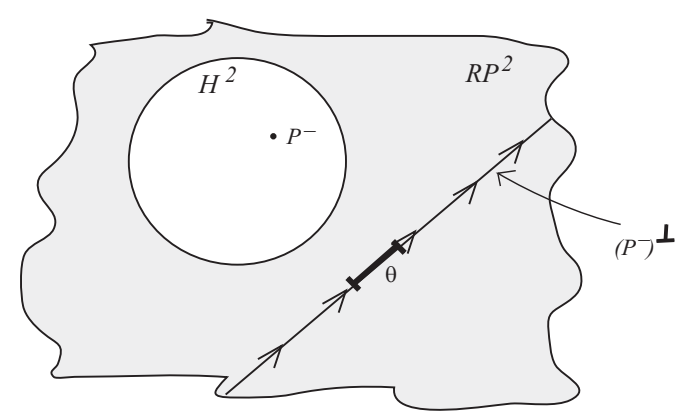

Figure 2. Action of $A=\cos \left(\frac{\theta}{2}\right)+\sin \left(\frac{\theta}{2}\right) P^{-}$in $R P^{2}$.

Case $2 N(A)=1, N\left(A^{-}\right)<0$. Then $A=\alpha+A^{-}, \alpha>1$. Thus $A=\cosh \left(\frac{d}{2}\right)+$ $\sinh \left(\frac{d}{2}\right) P^{-}$where $N\left(P^{-}\right)=-1$. The vector $P^{-}$belongs to the deSitter sphere, and we assume that its $Y$-coordinate is nonnegative. The vectors $A^{-}$and $P^{-}$are space-like vectors. Up to conjugation we have

$$
A=\cosh \left(\frac{d}{2}\right)+\sinh \left(\frac{d}{2}\right) J
$$

The matrix of the action of $c(A)$ as a linear map (see (2.3) ) on the basis $\{-I J, \quad J, \quad I\}$ of $E^{1,2}$ is:

$$
\begin{aligned}
m\left(-1,1 ; \cosh \left(\frac{d}{2}\right), 0, \sinh \left(\frac{d}{2}\right), 0\right)= & \\
= & =\left(\begin{array}{ccc}
\cosh ^{2}\left(\frac{d}{2}\right)+\sinh ^{2}\left(\frac{d}{2}\right) & 0 & 2 \cosh \left(\frac{d}{2}\right) \sinh \left(\frac{d}{2}\right) \\
0 & 1 & 0 \\
2 \cosh \left(\frac{d}{2}\right) \sinh \left(\frac{d}{2}\right) & 0 & \cosh ^{2}\left(\frac{d}{2}\right)+\sinh ^{2}\left(\frac{d}{2}\right)
\end{array}\right) \\
= & \left(\begin{array}{ccc}
\cosh (d) & 0 & -\sinh (d) \\
0 & 1 & 0 \\
-\sinh (d) & 0 & \cosh (d)
\end{array}\right)
\end{aligned}
$$

The action is a hyperbolic rotation around the oriented axis $P^{-}$. The action on the plane $\{-I J, I\} \subset E^{1,2}$, a plane orthogonal to $P^{-}=J$, is depicted in Figure 3 .

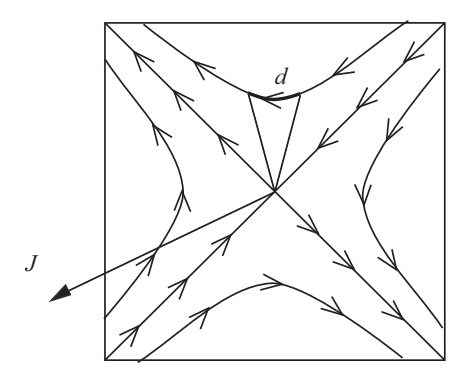

Figure 3. Action of $A=\cosh \left(\frac{d}{2}\right)+\sinh \left(\frac{d}{2}\right) J$ in $\{-I J, I\}$.

The action of $A=\cosh \left(\frac{d}{2}\right)+\sinh \left(\frac{d}{2}\right) J$ in $R P^{2}$ is depicted in Figure 4. 


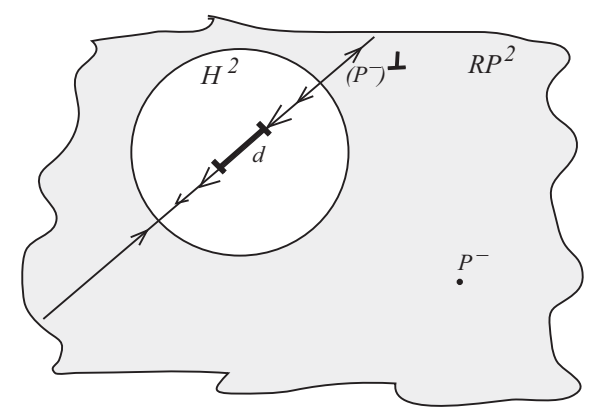

Figure 4 . The action of $A=\cosh \left(\frac{d}{2}\right)+\sinh \left(\frac{d}{2}\right) J$ in $R P^{2}$.

Case $3 N(A)=1, N\left(A^{-}\right)=0$. Then $A^{-}$belongs to the nullcone, it is a nullvector. Up to conjugation $A=1+I+J$. Then the matrix associated to $c(A)$ is

$$
m(-1,1 ; 1,1,1,0)=\left(\begin{array}{ccc}
1 & -2 & 2 \\
2 & -1 & 2 \\
2 & -2 & 3
\end{array}\right)
$$

The action is a parabolic transformation fixing $A^{-}$. See Figure 5 ,

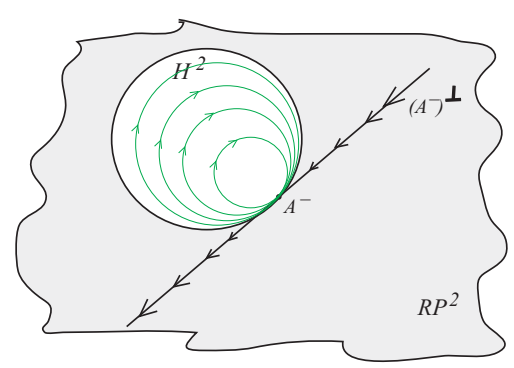

Figure 5. The action of $A=1+I+J$ in $R P^{2}$.

\subsection{Scalar and vector products on pure quaternions.}

Definition 3. The symmetric bilinear form $\langle,\rangle_{\mid H_{0}}$ defines the scalar product $s_{p}$ of two pure quaternions.

$$
\begin{array}{cccc}
s_{p}: & H_{0} \times H_{0} & \longrightarrow & k \\
& \left(A^{-}, B^{-}\right) & \rightarrow & \left\langle A^{-}, B^{-}\right\rangle
\end{array}
$$

where

$$
\left\langle A^{-}, B^{-}\right\rangle=\frac{1}{2}\left(A^{-} \overline{B^{-}}+B^{-} \overline{A^{-}}\right)=\frac{1}{2}\left(-A^{-} B^{-}-B^{-} A^{-}\right)=-\left(A^{-} B^{-}\right)^{+}
$$

Definition 4. The vector product $(\times)$ of two pure quaternions is given by

$$
\begin{aligned}
& \text { (×) } H_{0} \times H_{0} \rightarrow \quad H_{0} \\
& \left(A^{-}, B^{-}\right) \quad \rightarrow \quad A^{-} \times B^{-}:=\left(A^{-} B^{-}\right)^{-}
\end{aligned}
$$

Therefore the product $A^{-} B^{-}$of two pure quaternions decomposes as the difference between its vector product and its scalar product.

$$
A^{-} B^{-}=-\left\langle A^{-}, B^{-}\right\rangle+A^{-} \times B^{-}
$$


Lets compute the vector product of $A^{-}=x i+y j+z i j$, and $B^{-}=x^{\prime} i+y^{\prime} j+z^{\prime} i j$.

$$
\begin{aligned}
A^{-} \times B^{-} & =A^{-} B^{-}+\left\langle A^{-}, B^{-}\right\rangle= \\
= & (x i+y j+z i j)\left(x^{\prime} i+y^{\prime} j+z^{\prime} i j\right)+(x, y, z)\left(\begin{array}{ccc}
-\mu & 0 & 0 \\
0 & -\nu & 0 \\
0 & 0 & \mu \nu
\end{array}\right)\left(\begin{array}{c}
x^{\prime} \\
y^{\prime} \\
z^{\prime}
\end{array}\right) \\
& =-\nu\left(y z^{\prime}-y^{\prime} z\right) i-\mu\left(z x^{\prime}-z^{\prime} x\right) j+\left(x y^{\prime}-x^{\prime} y\right) i j=\left|\begin{array}{ccc}
-\nu i & -\mu j & i j \\
x & y & z \\
x^{\prime} & y^{\prime} & z^{\prime}
\end{array}\right|
\end{aligned}
$$

The above formula implies that the vector product is anticommutative:

$$
A^{-} \times B^{-}=-B^{-} \times A^{-} \text {. }
$$

Proposition 6. The vector product $A^{-} \times B^{-}$is orthogonal to the plane generated by $A^{-}$and $B^{-}$.

Proof. Suppose $A^{-}=x i+y j+z i j$, and $B^{-}=x^{\prime} i+y^{\prime} j+z^{\prime} i j$. Then

$$
\begin{aligned}
&\left\langle A^{-} \times B^{-}, A^{-}\right\rangle= \\
&=\left(-\nu\left(y z^{\prime}-y^{\prime} z\right),-\mu\left(z x^{\prime}-z^{\prime} x\right), x y^{\prime}-x^{\prime} y\right)\left(\begin{array}{ccc}
-\mu & 0 & 0 \\
0 & -\nu & 0 \\
0 & 0 & \mu \nu
\end{array}\right)\left(\begin{array}{l}
x \\
y \\
z
\end{array}\right)\left(\mu \nu x\left(y z^{\prime}-y^{\prime} z\right)+\mu \nu y\left(z x^{\prime}-z^{\prime} x\right)+\mu \nu z\left(x y^{\prime}-x^{\prime} y\right)\right)=0 ; \\
&\left\langle A^{-} \times B^{-}, B^{-}\right\rangle= \\
&=\left(-\nu\left(y z^{\prime}-y^{\prime} z\right),-\mu\left(z x^{\prime}-z^{\prime} x\right), x y^{\prime}-x^{\prime} y\right)\left(\begin{array}{ccc}
-\mu & 0 & 0 \\
0 & -\nu & 0 \\
0 & 0 & \mu \nu
\end{array}\right)\left(\begin{array}{l}
x^{\prime} \\
y^{\prime} \\
z^{\prime}
\end{array}\right) \\
&=\left(\mu \nu x^{\prime}\left(y z^{\prime}-y^{\prime} z\right)+\mu \nu y^{\prime}\left(z x^{\prime}-z^{\prime} x\right)+\mu \nu z^{\prime}\left(x y^{\prime}-x^{\prime} y\right)\right)=0
\end{aligned}
$$

Corollary 1. The pure quaternion $\left(A^{-} B^{-}\right)^{-}$is orthogonal to $A^{-}$and $B^{-}$.

\section{A PAIR OF CONJUGATE UNIT QUATERNIONS}

We are interested in subgroups of the group of unit quaternions generated by two conjugate elements. Therefore we shall analyze the properties of a pair of conjugate unit quaternions.

Lemma 1. Let $A, B \in U_{1}, A, B$ conjugate elements in $U_{1} \subset H=\left(\frac{\mu, \nu}{k}\right)$. Then $A^{+}=B^{+}$.

Proof. If $A, B$ are conjugate elements in $U_{1}$, there exist an element $C \in U_{1}$ such that

$$
C A C^{-1}=B
$$

Recall that for any quaternion $A \in H, T(A)=2 A^{+}$. Then it is enough to prove that $T(A)=T(B)$.

$$
\begin{aligned}
T(B) & =T\left(C A C^{-1}\right)=T(C A \bar{C})=C A \bar{C}+\overline{C A \bar{C}}=C A \bar{C}+C \bar{A} \bar{C} \\
& =C(A+\bar{A}) \bar{C}=C T(A) \bar{C}=T(A) C \bar{C}=T(A)
\end{aligned}
$$

Next we prove that the conjugation by a unit quaternion preserves the scalar product of pure quaternions. 
Lemma 2. Let $(A, B),\left(A_{0}, B_{0}\right)$ be two pairs of unit quaternions, that are conjugate (there exist a unit quaternion $C$ such that $C A \bar{C}=A_{0}$, and $C B \bar{C}=B_{0}$ ). Then $\left(A^{-} B^{-}\right)^{+}=\left(A_{0}^{-} B_{0}^{-}\right)^{+}$.

Proof. First note that $(C A \bar{C})^{-}=C A^{-} \bar{C}$. This is because

$$
C A \bar{C}=C\left(A^{+}+A^{-}\right) \bar{C}=A^{+}+C A^{-} \bar{C}
$$

and

yields to $(C A \bar{C})^{-}=C A^{-} \bar{C}$.

$$
C A \bar{C}=(C A \bar{C})^{+}+(C A \bar{C})^{-}=A^{+}+(C A \bar{C})^{-}
$$

Using this property, one obtain

$$
\begin{aligned}
\left(A_{0}^{-} B_{0}^{-}\right)^{+}=\left((C A \bar{C})^{-}(C B \bar{C})^{-}\right)^{+}=\left(C A^{-} \bar{C} C B^{-} \bar{C}\right)^{+} & \\
& =\left(C A^{-} B^{-} \bar{C}\right)^{+}=\left(A^{-} B^{-}\right)^{+}
\end{aligned}
$$

where the last equality is true by applying Lemma 1 to the conjugate quaternions $A^{-} B^{-}$and $C A^{-} B^{-} \bar{C}$.

The following two theorems show that a pair of conjugate (in $U_{1}$ ) unit quaternions in a quaternion algebra over $\mathbb{R}$ are determined, up to conjugation, by two real numbers: the trace of them and the scalar product of their pure parts. First we analyze the Hamilton quaternions.

Theorem 1. A pair $A, B$ of unit quaternions in $\mathbb{H}=\left(\frac{-1,-1}{\mathbb{R}}\right)$ with the same trace (2x) are determined, up to conjugation in $U_{1}$, by the real number $y=-\left(A^{-} B^{-}\right)^{+}$.

Proof. Write $A=x+A^{-}, B=x+B^{-}$. Note that

$$
\begin{aligned}
& N\left(A^{-}\right)=N\left(A-A^{+}\right)=N(A)-N\left(A^{+}\right)=1-x^{2} \\
& N\left(B^{-}\right)=N\left(B-B^{+}\right)=N(B)-N\left(B^{+}\right)=1-x^{2}
\end{aligned}
$$

implies that the pure quaternions $A^{-}$and $B^{-}$have the same norm, thus both are in the sphere of radius $\sqrt{1-x^{2}}$. Using conjugation by unit quaternions in $E^{3}\left(\cong H_{0}\right)$, Proposition [5] and Lemma 2, we can choose one pair $A, B$ with simpler coordinates as follows. Consider the coordinates system $\{X, Y, Z\}$ in $E^{3}$ in the basis $\{-i j, j, i\}$. First, conjugating by a unit quaternion $C$, such that $C B^{-} \bar{C}$ is in the positive $Z$ axis: $C B^{-} \bar{C}=\gamma i, \gamma>0$. Let $D$ be a unit quaternion such that the conjugation in $E^{3}\left(\cong H_{0}\right)$ by $D$ is a rotation around the $Z$-axis mapping $C A^{-} \bar{C}$ to a vector in the plane $Y Z$ with positive $Y$ - coordinate. See Figure [6. Then $D C B^{-} \overline{C D}=C B^{-} \bar{C}$ and $D C A \overline{C D}=\alpha i+\beta j, \beta \geqslant 0$.

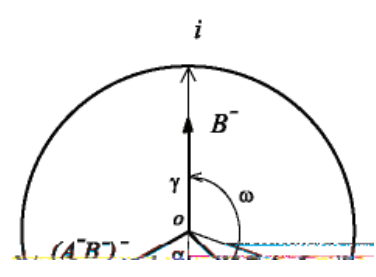

Figure 6 . The vectors $A^{-}$and $B^{-}$in $E^{3}$

Therefore we assume

$$
\begin{aligned}
& A^{-}=\alpha i+\beta j, \quad \beta \geqslant 0 \\
& B^{-}=\gamma i, \quad \gamma>0
\end{aligned}
$$


Note that

$$
\begin{aligned}
& N\left(A^{-}\right)=N\left((\alpha i+\beta j)=(\alpha i+\beta j)(-\alpha i-\beta j)=\left(\alpha^{2}+\beta^{2}\right)\right. \\
& N\left(B^{-}\right)=N(\gamma i)=(\gamma i)(-\gamma i)=\gamma^{2}
\end{aligned}
$$

Then

$$
\begin{aligned}
\alpha^{2}+\beta^{2} & =1-x^{2} \\
\gamma^{2} & =1-x^{2}
\end{aligned}
$$

From the computation of $A^{-} B^{-}$

$$
A^{-} B^{-}=(\alpha i+\beta j)(\gamma i)=-\gamma \alpha-\gamma \beta i j
$$

one obtains

$$
\begin{gathered}
y=-\left(A^{-} B^{-}\right)^{+}=\gamma \alpha \\
\left(A^{-} B^{-}\right)^{-}=-\gamma \beta i j
\end{gathered}
$$

Solving the equations $3.1,3.2$ and 3.3 we obtain

$$
\begin{aligned}
& \gamma=\sqrt{1-x^{2}} \\
& \alpha=\frac{y}{\gamma}=\frac{y}{\sqrt{1-x^{2}}} \\
& \beta=+\sqrt{1-x^{2}-\frac{y^{2}}{\gamma^{2}}}=\frac{1}{\sqrt{1-x^{2}}} \sqrt{\left(1-x^{2}\right)^{2}-y^{2}}
\end{aligned}
$$

Then, up to conjugation in $U_{1}$

$$
\begin{aligned}
& A=x+\frac{1}{+\sqrt{1-x^{2}}}\left(y i+\sqrt{\left(1-x^{2}\right)^{2}-y^{2}} j\right) \\
& B=x+\sqrt{1-x^{2}} i, \quad \sqrt{1-x^{2}}>0
\end{aligned}
$$

Observe also that

$$
\left(A^{-} B^{-}\right)^{-}=-\sqrt{\left(1-x^{2}\right)^{2}-y^{2}} i j
$$

The angle $\omega$ subtended by $A^{-}$and $B^{-}$(see Figure 6) is

$$
\cos \omega=\frac{\left\langle A^{-}, B^{-}\right\rangle}{\sqrt{\left\langle A^{-}, A^{-}\right\rangle} \sqrt{\left\langle B^{-}, B^{-}\right\rangle}}=\frac{-\left(A^{-} B^{-}\right)^{+}}{\gamma \gamma}=\frac{y}{1-x^{2}}
$$

Remark 2. The multiplicative group generated by the elements $A$ and $B$ 3.4) of the above Theorem 1 belongs to the quaternion algebra $\left(\frac{-1,-1}{k}\right)$, where

$$
k=Q\left(x, y, \sqrt{1-x^{2}}, \sqrt{\left(1-x^{2}\right)^{2}-y^{2}}\right)
$$

The case $M(2, \mathbb{R})=\left(\frac{-1,1}{\mathbb{R}}\right)$ is studied in the following theorem.

Theorem 2. A pair $A, B$ of conjugate (in $U_{1}$ ) independent unit quaternions in $M(2, \mathbb{R})=\left(\frac{-1,1}{\mathbb{R}}\right)$ with the same trace $(2 x)$ are determined, up to conjugation in $U_{ \pm 1}$, by the real number $y=-\left(A^{-} B^{-}\right)^{+}$.

Proof. Write $A=x+A^{-}, B=x+B^{-}$. There exists an element $C \in U_{1}$ such that

$$
C A \bar{C}=C\left(x+A^{-}\right) \bar{C}=x+C A^{-} \bar{C}=B=x+B^{-}
$$

Then, the vectors $A^{-}, B^{-} \in E^{1,2}$ are also conjugate

$$
C A^{-} \bar{C}=B^{-}
$$

The exact sequence (2.6) shows that conjugation by $C$ acts in $E^{1,2}$ as an element $c(C)$ of $\mathrm{SO}^{+}(1,2)$. 
Using conjugation and Lemma 2 we can choose one pair $A, B$ with convenient coordinates as follows.

Note that

$$
N\left(A^{-}\right)=N\left(B^{-}\right)=1-x^{2}
$$

Then we distinguish three cases according to the value of $N\left(A^{-}\right)(>,<,=) 0$, which geometrically fixes the position of $A^{-}$and $B^{-}$with respect to the nullcone. If we write $\{X, Y, Z\}$ to denote the coordinates in the basis $\{-I J, J, I\}, A^{-}$and $B^{-}$ belong to the hyperboloid

$$
-X^{2}-Y^{2}+Z^{2}=1-x^{2}=N\left(A^{-}\right)=N\left(B^{-}\right)
$$

which is a two-sheeted hyperboloid if $x^{2}<1$, the nullcone if $x^{2}=1$ and a onesheeted hyperboloid if $x^{2}>1$.

Case $1 N\left(A^{-}\right)=N\left(B^{-}\right)>0 . \quad\left(x^{2}=1-N\left(A^{-}\right)<1\right.$. Since $A^{-}$and $B^{-}$are conjugate by an element of $U_{1}$, they are placed in the same sheet of a twosheeted hyperboloid in the interior of the nullcone. Up to conjugation by $j \in U_{ \pm 1}$, if necessary, we assume that the time-like vectors $A^{-}$and $B^{-}$ are in the upper component of the hyperboloid. Because $S O^{+}(1,2)$ acts transitively on the rays inside the nullcone, we may assume that

$$
A^{-}=\gamma I, \quad \gamma>0
$$

A suitable conjugation by an elliptic rotation around the $Z$ axis, maps $B^{-}$ to a vector in the $Y Z$ plane with positive $Y$ coordinate. Say

$$
B^{-}=\alpha I+\beta J, \quad \alpha>\beta>0
$$

The following computations give the expressions of $\alpha, \beta$ and $\gamma$ as functions of $x$ and $y$ :

$$
\begin{gathered}
1 \Longrightarrow \begin{array}{c}
A \bar{A}=\left(A^{+}+A^{-}\right)\left(A^{+}-A^{-}\right)=x^{2}-A^{-} A^{-} \\
0<-A^{-} A^{-}=1-x^{2}=-(\gamma I)(\gamma I)=\gamma^{2}
\end{array} \\
\Longrightarrow \gamma=+\sqrt{1-x^{2}} \\
0<-B^{-} B^{-}=1-x^{2}=-(\alpha I+\beta J)(\alpha I+\beta J)=-\left(-\alpha^{2}+\beta^{2}\right) \\
\Longrightarrow \\
A^{-} B^{-}=(\gamma I)(\alpha I+\beta J)=-\gamma \alpha+\gamma \beta I J \\
\Longrightarrow \quad\left(A^{-} B^{-}\right)^{+}=-\gamma \alpha, \quad\left(A^{-} B^{-}\right)^{-}=\gamma \beta I J \\
\Longrightarrow y=\gamma \alpha
\end{gathered}
$$

Then, up to conjugation

$$
\begin{aligned}
& \begin{array}{c}
A=x+\sqrt{1-x^{2}} I, \quad \sqrt{1-x^{2}}>0 \\
B=x+\frac{1}{+\sqrt{1-x^{2}}}\left(y I+\sqrt{y^{2}-\left(1-x^{2}\right)^{2}} J\right)
\end{array} \\
& \left(A^{-} B^{-}\right)^{-}=+\sqrt{y^{2}-\left(1-x^{2}\right)^{2}} I J
\end{aligned}
$$

The hyperbolic distance $d$ between the projection of $A^{-}$and $B^{-}$on the hyperbolic plane (pure unit quaternions in the upper component) (see Figure (7) is

$$
\cosh d=\frac{\left\langle A^{-}, B^{-}\right\rangle}{\sqrt{\left\langle A^{-}, A^{-}\right\rangle} \sqrt{\left\langle B^{-}, B^{-}\right\rangle}}=\frac{-\left(A^{-} B^{-}\right)^{+}}{\gamma \gamma}=\frac{y}{1-x^{2}}
$$

The above quantity is larger than 1 and therefore is a hyperbolic cosine:

$$
\frac{y}{1-x^{2}}=\frac{\gamma \alpha}{\gamma^{2}}=\frac{\alpha}{\gamma} \text {, but } \gamma^{2}=1-x^{2}=\alpha^{2}-\beta^{2} \Longrightarrow \gamma^{2}<\alpha^{2} \Longrightarrow 0<\gamma<\alpha
$$




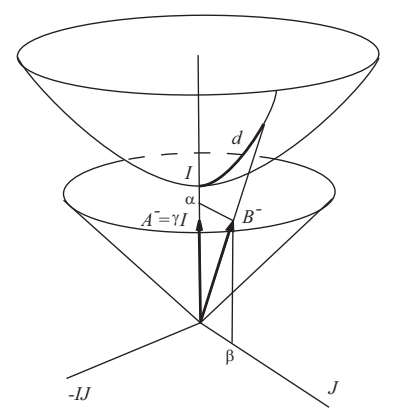

Figure 7 . The vectors $A^{-}, B^{-}$in $E^{1,2}$, case 1 .

Case $2 N\left(A^{-}\right)=N\left(B^{-}\right)<0 . \quad\left(x^{2}=1-N\left(A^{-}\right)>1\right)$. The vectors $A^{-}, B^{-}$are space-like vectors (outside the nullcone). Recall that $A^{-}, B^{-}$belong to a one-sheeted hyperboloid. There are three subcases according to the relative position of the plane $\Pi$ defined by $A^{-}, B^{-}$and the nullcone. We shall prove that these cases correspond to $y^{2}(>,<,=) \gamma^{4}$.

Subcase 2A The plane $\Pi$ intersects the nullcone. See Figure 8 Considering the projective plane $R P^{2}$ defined by the lines through the origin in $E^{1,2}$, the nullcone defines a conic $\mathcal{C}$. The polars of $A^{-}$and $B^{-}$with respect to the conic $\mathcal{C}$ intersect in a point $Q$ (pole of $\Pi$ ) outside $\mathcal{C}$. Up to conjugation we can suppose that $Q=\infty$. Then there are two possibilities : $A^{-}, B^{-}$are either on the same side or on different side of $\mathcal{C}$. See Figure 9 for the situation in $E^{1,2}$.

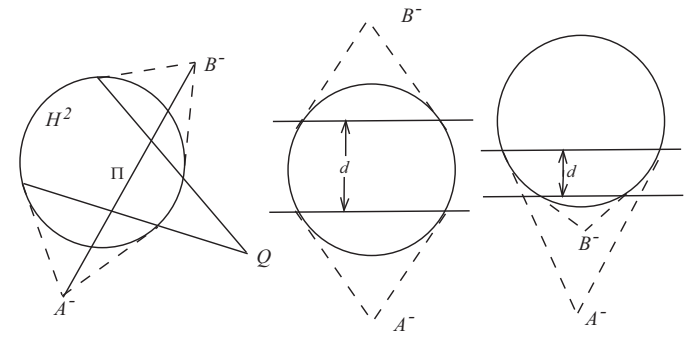

Figure 8. Subcase 2A.

Up to conjugation

$$
\begin{aligned}
& A^{-}=\gamma J, \quad \gamma>0 \\
& B^{-}=\alpha I+\beta J, \quad\left\{\begin{array}{l}
\beta>\alpha>0 \\
\beta<\alpha<0
\end{array}\right\}
\end{aligned}
$$

The following computations give the expressions of $\alpha, \beta$ and $\gamma$ as functions of $x$ and $y$ :

$$
\begin{gathered}
0>N\left(A^{-}\right)=-A^{-} A^{-}=1-x^{2}=-(\gamma J)(\gamma J)=-\gamma^{2} \\
0>N\left(B^{-}\right)=-B^{-} B^{-}=1-x^{2}=-(\alpha I+\beta J)(\alpha I+\beta J)=-\left(-\alpha^{2}+\beta^{2}\right) \\
\Longrightarrow \begin{array}{c}
\gamma=+\sqrt{x^{2}-1} \\
-\alpha^{2}+\beta^{2}=x^{2}-1
\end{array} \\
A^{-} B^{-}=(\gamma J)(\alpha I+\beta J)=\gamma \beta-\gamma \alpha I J \\
\Longrightarrow\left(A^{-} B^{-}\right)^{+}=\gamma \beta, \quad\left(A^{-} B^{-}\right)^{-}=-\gamma \alpha I J
\end{gathered}
$$




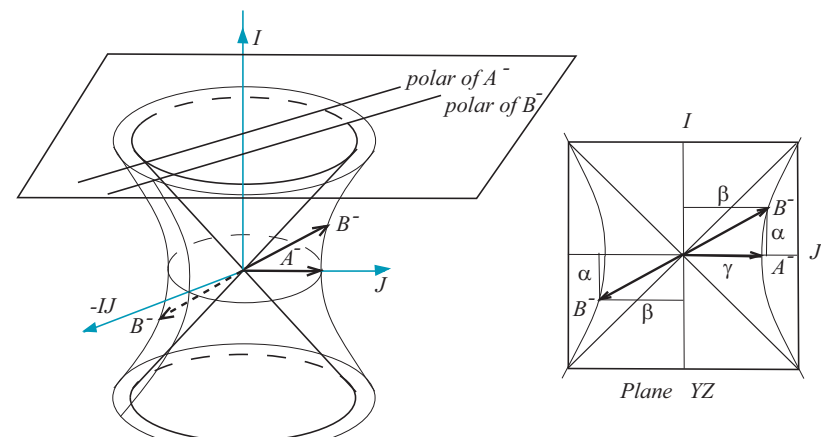

Figure 9. The vectors $A^{-}, B^{-}$in $E^{1,2}$, subcase $2 \mathrm{~A}$.

$$
\begin{gathered}
\Longrightarrow y=-\gamma \beta \\
\Longrightarrow\left\{\begin{array}{l}
y<0, \beta=\frac{y}{-\gamma}>0, \Longrightarrow \alpha>0 \Longrightarrow \alpha=+\frac{1}{\gamma} \sqrt{y^{2}-\gamma^{4}} \\
y>0, \beta=\frac{y}{-\gamma}<0, \Longrightarrow \alpha<0 \Longrightarrow \alpha=-\frac{1}{\gamma} \sqrt{y^{2}-\gamma^{4}}
\end{array}\right\}
\end{gathered}
$$

Therefore, the two possibilities are given by the sign of $y$. For $y<0$.

$$
\begin{gathered}
A=x+\sqrt{x^{2}-1} J, \quad \sqrt{x^{2}-1}>0 \\
B=x+\frac{1}{+\sqrt{x^{2}-1}}\left(\sqrt{y^{2}-\left(x^{2}-1\right)^{2}} I-y J\right) \\
\left(A^{-} B^{-}\right)^{-}=-\sqrt{y^{2}-\left(x^{2}-1\right)^{2}} I J \\
\cosh d=\frac{-y}{x^{2}-1}
\end{gathered}
$$

For $y>0$.

$$
\begin{gathered}
A=x+\sqrt{x^{2}-1} J, \quad \sqrt{x^{2}-1}>0 \\
B=x-\frac{1}{+\sqrt{x^{2}-1}}\left(\sqrt{y^{2}-\left(x^{2}-1\right)^{2}} I+y J\right) \\
\left(A^{-} B^{-}\right)^{-}=+\sqrt{y^{2}-\left(x^{2}-1\right)^{2}} I J \\
\cosh d=\frac{y}{x^{2}-1}
\end{gathered}
$$

Where $d$ is the distance between the polars of $A^{-}$and $B^{-}$. Note that

$$
\cosh ^{2} d=\frac{\left\langle A^{-}, B^{-}\right\rangle^{2}}{\left\langle A^{-}, A^{-}\right\rangle\left\langle B^{-}, B^{-}\right\rangle}=\frac{y^{2}}{\left(x^{2}-1\right)^{2}}>1 \Longleftrightarrow y^{2}>\gamma^{4}
$$

because the polars of $A^{-}$and $B^{-}$do not intersect inside the conic.

Subcase 2B The plane $\Pi$ does not intersect the nullcone. See Figure 10 for the situation in projective plane $R P^{2}$ defined by the lines through the origin in $E^{1,2}$. The polars of $A^{-}$and $B^{-}$with respect to the conic $\mathcal{C}$ intersect in a point $Q$ (pole of $\Pi$ ) inside $\mathcal{C}$.

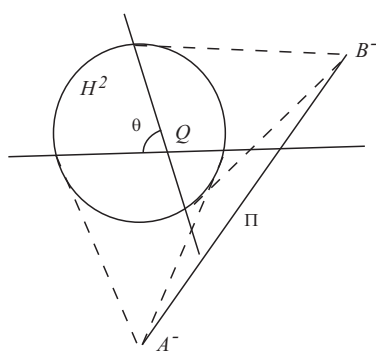

Figure 10. Subcase 2B. 
Up to conjugation we can suppose that $Q$ is the center of the conic $\mathcal{C}$. The angle $\theta$ is given by

$$
\cos \theta=\frac{\left\langle A^{-}, B^{-}\right\rangle}{\sqrt{\left\langle A^{-}, A^{-}\right\rangle} \sqrt{\left\langle B^{-}, B^{-}\right\rangle}}
$$

See Figure 11 for the situation in $E^{1,2}$.
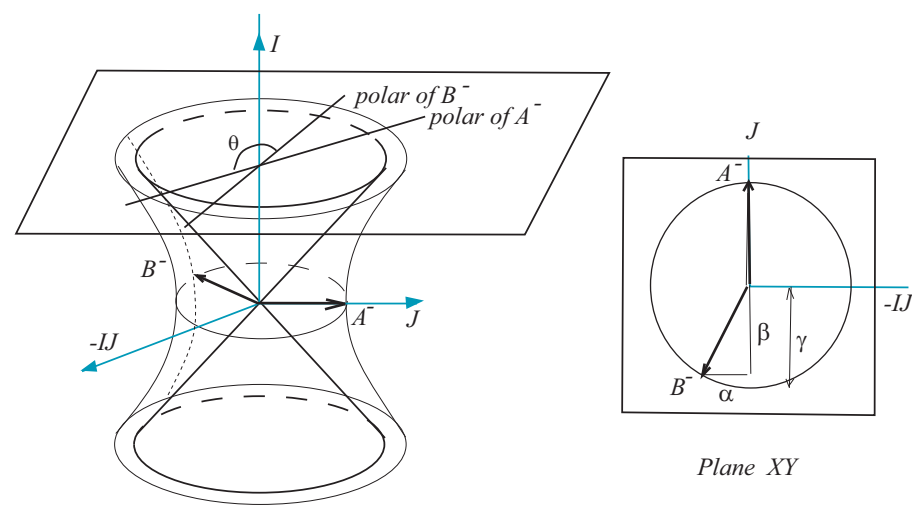

Plane $X Y$

Figure 11. The vectors $A^{-}, B^{-}$in $E^{1,2}$, subcase $2 \mathrm{~B}$.

The vectors $A^{-}$and $B^{-}$are in the $X Y$ plane. Up to conjugation,

$$
\begin{aligned}
& A^{-}=\gamma J, \quad \gamma>0 \\
& B^{-}=\alpha I J+\beta J
\end{aligned}
$$

Then

$$
\begin{aligned}
& 0>N\left(A^{-}\right)=-A^{-} A^{-}=1-x^{2}=-(\gamma J)(\gamma J)=-\gamma^{2} \\
& 0>N\left(B^{-}\right)=-B^{-} B^{-}=1-x^{2}=-(\alpha I J+\beta J)(\alpha I J+\beta J)=-\alpha^{2}-\beta^{2} \\
& \Longrightarrow \begin{array}{c}
\gamma=+\sqrt{x^{2}-1} \\
\alpha^{2}+\beta^{2}=x^{2}-1
\end{array} \\
& A^{-} B^{-}=(\gamma J)(\alpha I J+\beta J)=-\gamma \alpha I+\gamma \beta \\
& \Longrightarrow\left(A^{-} B^{-}\right)^{+}=\gamma \beta, \quad\left(A^{-} B^{-}\right)^{-}=-\gamma \alpha I \\
& \Longrightarrow y=-\gamma \beta \\
& \cos ^{2} \theta=\frac{\left\langle A^{-}, B^{-}\right\rangle^{2}}{\left\langle A^{-}, A^{-}\right\rangle\left\langle B^{-}, B^{-}\right\rangle}=\frac{y^{2}}{\left(x^{2}-1\right)^{2}}<1 \Longleftrightarrow y^{2}<\left(x^{2}-1\right)^{2}=\gamma^{4} \\
& \beta=\frac{-y}{\sqrt{x^{2}-1}} \\
& \alpha^{2}=x^{2}-1-\beta^{2}=\frac{\left(x^{2}-1\right)^{2}-y^{2}}{\left(x^{2}-1\right)}>0 \\
& \alpha= \pm \sqrt{\frac{\left(x^{2}-1\right)^{2}-y^{2}}{\left(x^{2}-1\right)}}
\end{aligned}
$$

There are two possibilities for $\alpha$. See Figure 12 .

Conjugation by $J \in U_{ \pm 1}$, acts in $A^{-}, B^{-}$as follows. Note that $J^{2}=$ $1 \Longrightarrow J^{-1}=J$.

$$
J\left(A^{-}, B^{-}\right) J=(J \gamma J J, J(\alpha I J+\beta J) J)=(\gamma J,-\alpha I J+\beta J)
$$



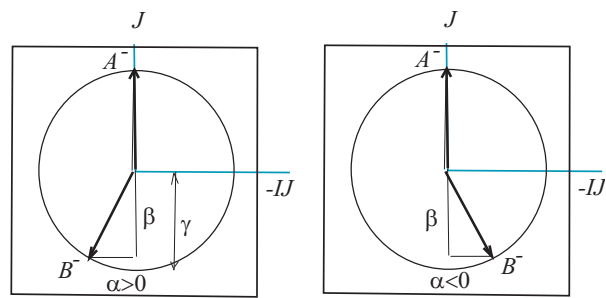

Plane $\Pi$

Figure 12. The two possibilities for $\alpha$ in the plane $X Y$.

Therefore, up to conjugation we can choose $\alpha>0$. Then, in this subcase

$$
\begin{gathered}
A=x+\sqrt{x^{2}-1} J, \quad \sqrt{x^{2}-1}>0 \\
B=x+\frac{1}{+\sqrt{x^{2}-1}}\left(-y J+\sqrt{\left(x^{2}-1\right)^{2}-y^{2}} I J\right) \\
\left(A^{-} B^{-}\right)^{-}=-\sqrt{\left(x^{2}-1\right)^{2}-y^{2}} I J \\
\cos \theta=\frac{-y}{x^{2}-1} \Longrightarrow\left\{\begin{array}{l}
y<0 \Longleftrightarrow 0<\theta<\frac{\pi}{2} \\
y>0 \Longleftrightarrow \frac{\pi}{2}<\theta<\pi
\end{array}\right\}
\end{gathered}
$$

Subcase $2 \mathrm{C}$ The plane $\Pi$ is tangent to the nullcone. See Figure 13. Assume $\Pi$ is tangent to $\mathcal{N}$ and contains the $Y$ axis. Then, we can assume that $A^{-}, B^{-}$are in the plane $X=Z$, where $\{X, Y, Z\}$ correspond to coordinates in the basis $\{-I J, J, I\}$.
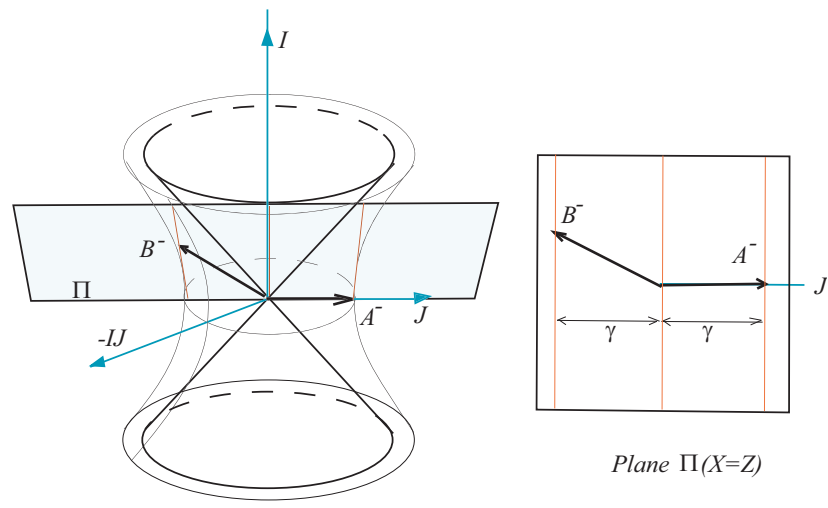

Plane $\Pi(X=Z)$

Figure 13. Subcase 2C.

Up to conjugation by a parabolic transformation fixing $\Pi$, we may assume that

$$
\begin{aligned}
& A^{-}=\gamma J, \quad \gamma>0 \\
& B^{-}=\lambda(I J+I)+\gamma^{\prime} J
\end{aligned}
$$

Then

$$
\begin{aligned}
0>N\left(A^{-}\right) & =-A^{-} A^{-}=1-x^{2}=-(\gamma J)(\gamma J)=-\gamma^{2} \\
0>N\left(B^{-}\right) & =-B^{-} B^{-}=1-x^{2}=-\left(\lambda(I J+I)+\gamma^{\prime} J\right)\left(\lambda(I J+I)+\gamma^{\prime} J\right) \\
& =-\left(\gamma^{\prime}\right)^{2}
\end{aligned}
$$

implies that $\gamma^{\prime}= \pm \gamma$, then we write $\gamma^{\prime}=\varepsilon \gamma$, where $\varepsilon= \pm 1$. This also can be obtained geometrically, because $A^{-}, B^{-}$are both in the one-sheeted 
hyperboloid $-X^{2}-Y^{2}+Z^{2}=1-x^{2}=N\left(A^{-}\right)=N\left(B^{-}\right)=-\gamma^{2}$. Then, $A^{-}, B^{-}$are in

$$
\left\{\begin{array}{c}
-X^{2}-Y^{2}+Z^{2}=-\gamma^{2} \\
X=Z
\end{array}\right\}=\left\{\begin{array}{c}
Y^{2}=\gamma^{2} \\
X=Z
\end{array}\right\}=\left\{\begin{array}{c}
Y= \pm \gamma \\
X=Z
\end{array}\right\}
$$

Assume $\lambda>0$, then the conjugation by the unit quaternion

$$
C=\frac{1+\lambda}{2 \sqrt{\lambda}}+\frac{\lambda-1}{2 \sqrt{\lambda}} J
$$

acts in $H_{0}$ as a hyperbolic rotation about the Y-axis $J$. See Figure 3 . The matrix of this transformation is

$$
c(C)=\left(\begin{array}{ccc}
\frac{1+\lambda^{2}}{2 \lambda} & 0 & \frac{1-\lambda^{2}}{2 \lambda} \\
0 & 1 & 0 \\
\frac{1-\lambda^{2}}{2 \lambda} & 0 & \frac{1+\lambda^{2}}{2 \lambda}
\end{array}\right)
$$

Therefore

$$
\begin{aligned}
c(C)(\gamma J) & =\gamma J \\
c(C)(\lambda(I J+I)+\varepsilon \gamma J) & =(I J+I)+\varepsilon \gamma J
\end{aligned}
$$

If $\lambda<0$ conjugation by

$$
D=\frac{1-\lambda}{2 \sqrt{-\lambda}}+\frac{-\lambda-1}{2 \sqrt{-\lambda}} J
$$

acts as

$$
\begin{aligned}
c(D)(\gamma J) & =\gamma J \\
c(D)(\lambda(I J+I)+\varepsilon \gamma J) & =(I J+I)+\varepsilon \gamma J
\end{aligned}
$$

Then, up to conjugation, we can assume

$$
\begin{aligned}
& A^{-}=\gamma J, \quad \gamma>0 \\
& B^{-}=(I J+I)+\gamma^{\prime} J
\end{aligned}
$$

To relate the parameters to $x, y$, we compute as usual

$$
\begin{gathered}
A^{-} B^{-}=(\gamma J)((I J+I)+\varepsilon \gamma J)=-\gamma I-\gamma I J+\gamma^{2} \varepsilon \\
\Longrightarrow \quad\left(A^{-} B^{-}\right)^{+}=\gamma^{2} \varepsilon, \quad\left(A^{-} B^{-}\right)^{-}=-\gamma I-\gamma I J \\
\Longrightarrow y=-\varepsilon \gamma^{2} \\
\varepsilon=\frac{-y}{\gamma^{2}}, \quad \gamma^{\prime}=-\frac{y}{\gamma} \quad \text { and } \quad y^{2}=\gamma^{4}
\end{gathered}
$$

Therefore

$$
\begin{gathered}
A=x+\sqrt{x^{2}-1} J, \quad \sqrt{x^{2}-1}>0 \\
B=x+(I J+I)-\frac{y}{\sqrt{x^{2}-1}} J \\
\left(A^{-} B^{-}\right)^{-}=-\sqrt{\left(x^{2}-1\right)}(I+I J)
\end{gathered}
$$

Case $3 N\left(A^{-}\right)=N\left(B^{-}\right)=0 .\left(x^{2}=1\right)$. The vectors $A^{-}, B^{-}$are in the nullcone. Since $A^{-}, B^{-}$are conjugate in $U_{1}$, they belong to the same component of $\mathcal{N}-(0)$. Conjugating by $J \in U_{ \pm 1}$ if necessary, assume $A^{-}, B^{-} \subset \mathcal{N}_{+}$. Up to conjugation by a rotation around $I$ sending $A^{-}$to $\gamma(I+J)$, and a parabolic around $A^{-}$, we can assume that $A^{-}, B^{-}$are as follows:

$$
A^{-}=\gamma(I+J), \quad B^{-}=\alpha(I-J)
$$

By using a hyperbolic rotation around $I J$, we can suppose also that $\gamma=1$. See Figure 14. 


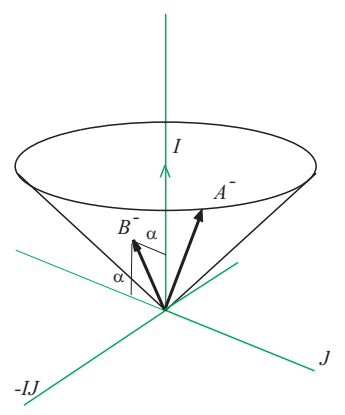

Figure 14. Case 3.

Then

$$
\begin{gathered}
A^{-} B^{-}=\alpha(I+J)(I-J)=-2 \alpha-2 \alpha I J \\
\Longrightarrow \quad\left(A^{-} B^{-}\right)^{+}=-2 \alpha, \quad\left(A^{-} B^{-}\right)^{-}=-2 \alpha I J \\
\Longrightarrow y=2 \alpha
\end{gathered}
$$

Thus

$$
\begin{gathered}
A=x+I+J \\
B=x+\frac{y}{2}(I-J) \\
\left(A^{-} B^{-}\right)^{-}=-y I J
\end{gathered}
$$

Remark 3. The multiplicative group generated by the elements $A$ and $B$ of the above theorem 2 belong to a quaternion algebra $\left(\frac{-1,1}{k}\right)$, according to the following cases:

Case $1 A$ and $B$ in (3.5) $k=Q\left(x, y, \sqrt{1-x^{2}}, \sqrt{y^{2}-\left(1-x^{2}\right)^{2}}\right)$

Subcase $2 \mathrm{~A} A$ and $B$ in (3.6) and (3.7) $k=Q\left(x, y, \sqrt{x^{2}-1}, \sqrt{y^{2}-\left(x^{2}-1\right)^{2}}\right)$

Subcase $2 \mathrm{~B} A$ and $B$ in (3.8) $k=Q\left(x, y, \sqrt{x^{2}-1}, \sqrt{\left(x^{2}-1\right)^{2}-y^{2}}\right)$

Subcase $2 \mathrm{C} A$ and $B$ in (3.9) $k=Q\left(x, y, \sqrt{x^{2}-1}\right)$

Case $3 A$ and $B$ in (3.10) $k=Q(x, y)$

Proposition 7. Let $A, B \in U_{1}$ be conjugate elements in $U_{1}$, the unit quaternions of a quaternion algebra $H=\left(\frac{\mu, \nu}{k}\right)$. The subalgebra of $H$ generated (as an $k$-algebra) by $A$ and $B$ coincides with the $k$-vector subspace of $H$ generated by

$$
\left\{1, A^{-}, B^{-},\left(A^{-} B^{-}\right)^{-}\right\} \text {. }
$$

Proof. Set $A=A^{+}+A^{-}, B=B^{+}+B^{-}$. Then, since $A$ and $B$ are conjugate, $A^{+}=B^{+}$(Lemma 1). Set $x=A^{+}=B^{+} \in k$. Take a word $r(A, B)$ in the alphabet $\left\{A, B, A^{-1}, B^{-1}\right\}$. Since $A, B \in U_{1}, A^{-1}=\bar{A}$ and $B^{-1}=\bar{B}$. Then because

$$
\begin{aligned}
& A=x+A^{-} \\
& \bar{A}=x-A^{-} \\
& B=x+B^{-} \\
& \bar{B}=x-B^{-}
\end{aligned}
$$

$r(A, B)$ is a finite product of the expressions $\left(x+A^{-}\right),\left(x-A^{-}\right),\left(x+B^{-}\right),\left(x-B^{-}\right)$. This is a $k$-linear combination of words $s\left(A^{-}, B^{-}\right)$in the alphabet $\left\{A^{-}, B^{-}\right\}$. Using the formulas

$$
\begin{gathered}
2\left(A^{-} B^{-}\right)^{+}=A^{-} B^{-}+B^{-} A^{-} \in k \\
A^{-} A^{-}=B^{-} B^{-}=x^{2}-1 \in k
\end{gathered}
$$


it is possible to write $s\left(A^{-}, B^{-}\right)$as a $k$-linear combination of $\left\{1, A^{-}, B^{-}, A^{-} B^{-}\right\}$. Finally, note that $A^{-} B^{-}=\left(A^{-} B^{-}\right)^{+}+\left(A^{-} B^{-}\right)^{-}$.

Definition 5. Let $A, B \in U_{1}$ be conjugate elements in $U_{1}$, the unit quaternions of the quaternion algebra $H=\frac{(\mu, \nu)}{k}$. We say that $(A, B)$ is an irreducible pair iff $A$, $B$ generates $H$ as a $k$-algebra. Otherwise we say that $(A, B)$ is reducible. Thus $(A, B)$ is irreducible iff

$$
\mathcal{B}=\left\{1, A^{-}, B^{-},\left(A^{-} B^{-}\right)^{-}\right\}
$$

is a basis of the $k$-vector space $H$. We will say that $(A, B)$ is almost-irreducible iff $(A, B)$ is reducible but $A^{-}$and $B^{-}$are linearly independent.

\section{C-REPRESENTATIONS IN $U_{1}$}

Let $G$ be a group given by the presentation

$$
G=|a, b: w(a, b)|
$$

where $w$ is a word in $a$ and $b$.

A homomorphism

$$
\rho: G \longrightarrow U_{1}
$$

such that $\rho(a)$ and $\rho(b)$ are conjugate elements in $U_{1}$ is called here a c-representation. Set

$$
\rho(a)=A=A^{+}+A^{-}, \quad \rho(b)=B=B^{+}+B^{-} .
$$

Then, by Lemma 1 $A^{+}=B^{+}$since $A$ and $B$ are conjugate elements. Set

$$
x=A^{+}=B^{+}
$$

and

$$
y=-\left(A^{-} B^{-}\right)^{+} .
$$

Definition 6. We will say that a c-representation $\rho: G \longrightarrow U_{1}$ of a two generator group $G=|a, b: w(a, b)|$ realizes the point $(x, y)$ if $x=\rho(a)^{+}=\rho(b)^{+}$and $y=$ $-\left(\rho(a)^{-} \rho(b)^{-}\right)^{+}$.

By Proposition 7 the subalgebra of $H$ generated (as a $k$-algebra) by $i m(\rho)$ coincides with the $k$-vector subspace of $H$ generated (as a $k$-vector space) by

$$
\left\{1, A^{-}, B^{-},\left(A^{-} B^{-}\right)^{-}\right\}
$$

We say that $\rho$ is irreducible iff $i m(\rho)$ generates $H$ as a $k$-algebra. Otherwise we say that $\rho$ is reducible. Thus $\rho$ is irreducible iff $(A, B)$ is an irreducible pair iff

$$
\mathcal{B}=\left\{1, A^{-}, B^{-},\left(A^{-} B^{-}\right)^{-}\right\}
$$

is a basis of the $k$-vector space $H$.

We will say that $\rho$ is almost-irreducible iff $(A, B)$ is an almost-irreducible pair; that is: $\rho$ is reducible but $A^{-}$and $B^{-}$are linearly independent.

Proposition 8. A c-representation

$$
\rho: G=|a, b: w(a, b)| \longrightarrow U_{1}
$$

is almost-irreducible iff $\rho$ is a reducible metabelian c-representation.

Proof. Recall that a representation is called reducible metabelian if the image $\rho(G)$ is reducible but it is not an abelian subgroup in $U_{1}$. See $[9$. On the other hand it is clear that the subgroup generate by $A$ and $B$ is abelian if and only if the two pure quaternions $A^{-}$and $B^{-}$commute, and this occurs if and only if $A^{-}= \pm B^{-}$. 
Theorem 3. Let $G$ be a group given by the presentation

$$
G=|a, b: w(a, b)|
$$

where $w$ is a word in $a$ and $b$ and let $U_{1}$ denote the group of unit quaternions of the algebra $\left(\frac{-1,1}{\mathbb{C}}\right)$. Then there is an algorithm to construct an ideal $\mathcal{I}_{G}^{c}$ generated by four polynomials

$$
\left\{p_{1}(x, y), p_{2}(x, y), p_{3}(x, y), p_{4}(x, y)\right\}
$$

with integer coefficients that can be characterized as follows:

(1) The set of points $\left\{(x, y) \in V\left(\mathcal{I}_{G}^{c}\right): y^{2} \neq\left(1-x^{2}\right)^{2}\right\}$ coincides with the pairs $(x, y)$ for which there exists an irreducible c-representation $\rho: G \longrightarrow U_{1}$, unique up to conjugation in $U_{1}$, realizing $(x, y)$.

(2) The set of points $\left\{(x, y) \in V\left(\mathcal{I}_{G}^{c}\right): y^{2}=\left(1-x^{2}\right)^{2}, \quad x^{2} \neq 1\right\}$ coincides with the pairs $(x, y)$ for which there exists an almost-irreducible c-representation $\rho: G \longrightarrow U_{1}$, unique up to conjugation in $U_{1}$, realizing $(x, y)$.

(3) The set of points $\left\{(x, y) \in V\left(\mathcal{I}_{G}^{c}\right): y=0, \quad x^{2}=1\right\}$ coincides with the pairs $(x, y)$ for which neither irreducible nor almost-irreducible c-representation $\rho: G \longrightarrow U_{1}$ realizing $(x, y)$ exist.

Proof. Let $\rho: G \longrightarrow U_{1}$ be an irreducible c-representation. Set $\rho(a)=A$ and $\rho(b)=B$ and

$$
\begin{gathered}
x=A^{+}=B^{+} \\
y=-\left(A^{-} B^{-}\right)^{+}
\end{gathered}
$$

and consider the vector basis

$$
\mathcal{B}=\left\{1, A^{-}, B^{-},\left(A^{-} B^{-}\right)^{-}\right\} .
$$

Then

$$
\begin{aligned}
1 & =A A^{-1}=A \bar{A}=\left(A^{+}+A^{-}\right)\left(A^{+}-A^{-}\right)=x^{2}-A^{-} A^{-} \\
& \Longrightarrow \quad 1-x^{2}=-A^{-} A^{-}=-B^{-} B^{-}
\end{aligned}
$$

Set:

$$
u=1-x^{2}
$$

The left product by $X \in H$ can be considered as a linear automorphism of the 4-dimensional vector space $H$.

$$
\begin{aligned}
& \mathbb{X}: H \longrightarrow H \\
& C \rightarrow X C
\end{aligned}
$$

We want to write the matrix of $\mathbb{X}$ with respect to the basis

$$
\mathcal{B}=\left\{1, A^{-}, B^{-},\left(A^{-} B^{-}\right)^{-}\right\}
$$

belonging to the $k$-vector space structure of the quaternion algebra $H$. Denote by $I_{4 \times 4}$ the identity matrix 4 , and by $m(X)$ the matrix of the automorphism $\mathbb{X}$ with respect to $\mathcal{B}$. Observe that

$$
\begin{aligned}
& m(A)=x I_{4 \times 4}+m\left(A^{-}\right) \\
& m(\bar{A})=x I_{4 \times 4}-m\left(A^{-}\right) \\
& m(B)=x I_{4 \times 4}+m\left(B^{-}\right) \\
& m(\bar{B})=x I_{4 \times 4}-m\left(B^{-}\right)
\end{aligned}
$$

Computation of $m\left(A^{-}\right)$:

$$
\begin{aligned}
& A^{-} A^{-}=-u \\
& A^{-} B^{-}=\left(A^{-} B^{-}\right)^{+}+\left(A^{-} B^{-}\right)^{-}=-y+\left(A^{-} B^{-}\right)^{-}
\end{aligned}
$$


From the last equation

$$
\left(A^{-} B^{-}\right)^{-}=y+A^{-} B^{-}
$$

Then

$$
A^{-}\left(A^{-} B^{-}\right)^{-}=A^{-}\left(y+A^{-} B^{-}\right)=y A^{-}+A^{-} A^{-} B^{-}=y A^{-}-u B^{-}
$$

$$
m\left(A^{-}\right)=\left(\begin{array}{cccc}
0 & -u & -y & 0 \\
1 & 0 & 0 & y \\
0 & 0 & 0 & -u \\
0 & 0 & 1 & 0
\end{array}\right)
$$

Computation of $m\left(B^{-}\right)$: Recall that $2\left(A^{-} B^{-}\right)^{+}=A^{-} B^{-}+B^{-} A^{-}$, then

$$
\begin{aligned}
B^{-} A^{-} & =2\left(A^{-} B^{-}\right)^{+}-A^{-} B^{-}=-2 y-\left(-y+\left(A^{-} B^{-}\right)^{-}\right) \\
& =-y-\left(A^{-} B^{-}\right)^{-} \\
B^{-} B^{-} & =-u \\
B^{-}\left(A^{-} B^{-}\right)^{-} & =B^{-}\left(y+A^{-} B^{-}\right)=y B^{-}+B^{-} A^{-} B^{-} \\
& =y B^{-}+\left(2\left(A^{-} B^{-}\right)^{+}-A^{-} B^{-}\right) B^{-} \\
& =y B^{-}-2 y B^{-}+u A^{-}=-y B^{-}+u A^{-}
\end{aligned}
$$

$$
m\left(B^{-}\right)=\left(\begin{array}{cccc}
0 & -y & -u & 0 \\
0 & 0 & 0 & u \\
1 & 0 & 0 & -y \\
0 & -1 & 0 & 0
\end{array}\right)
$$

Computation of $m\left(\left(A^{-} B^{-}\right)^{-}\right)$:

$$
\begin{aligned}
\left(A^{-} B^{-}\right)^{-} A^{-} & =\left(y+A^{-} B^{-}\right) A^{-}=\left(y+2\left(A^{-} B^{-}\right)^{+}-B^{-} A^{-}\right) A^{-} \\
& =-y A^{-}+u B^{-} \\
\left(A^{-} B^{-}\right)^{-} B^{-} & =\left(y+A^{-} B^{-}\right) B^{-}=y B^{-}-u A^{-} \\
\left(A^{-} B^{-}\right)^{-}\left(A^{-} B^{-}\right)^{-} & =\left(-y-B^{-} A^{-}\right)\left(y+A^{-} B^{-}\right) \\
& =-y^{2}-y\left(B^{-} A^{-}+A^{-} B^{-}\right)-u^{2}=y^{2}-u^{2}
\end{aligned}
$$

$$
\left.m\left(A^{-} B^{-}\right)^{-}\right)=\left(\begin{array}{cccc}
0 & 0 & 0 & y^{2}-u^{2} \\
0 & -y & -u & 0 \\
0 & u & y & 0 \\
1 & 0 & 0 & 0
\end{array}\right)
$$

Observe that $\mathbb{X}(1)=X$. Therefore, if $m(X)$ is the matrix of the linear map $\mathbb{X}$ then the coordinates of the quaternion $X$ with respect to $\mathcal{B}$ is the 4 -tuple

$$
m(X)\left(\begin{array}{l}
1 \\
0 \\
0 \\
0
\end{array}\right)
$$

Next we use the relator of the presentation $|a, b: w(a, b)|$ to obtain necessary conditions on $x, y$ for $\rho: G \longrightarrow U_{1}$ be a homomorphism. Computing the matrix $w(m(A), m(B))$ and imposing the condition that it is the matrix with respect to $\mathcal{B}$ of the "product by 1 " linear map $H \longrightarrow H$, a $4 \times 1$ matrix

$$
w(m(A), m(B))\left(\begin{array}{l}
1 \\
0 \\
0 \\
0
\end{array}\right)-\left(\begin{array}{l}
1 \\
0 \\
0 \\
0
\end{array}\right)
$$


is obtained whose entries form a set $\left\{p_{1}(x, y), p_{2}(x, y), p_{3}(x, y), p_{4}(x, y)\right\}$ of four polynomials with integer coefficients such that $p_{i}(x, y)=0, \quad i=\{1,2,3,4\}$. This proves the first part of the theorem.

It is clear that if given $x_{0} \in \mathbb{C}, y_{0} \in \mathbb{C}$ such that $p_{i}\left(x_{0}, y_{0}\right)=0$ for $i=\{1,2,3,4\}$, we can find quaternions $A$ and $B$ in $U_{1} \subset\left(\frac{-1,1}{\mathbb{C}}\right)$ such that

$$
\begin{gathered}
x_{0}=A^{+}=B^{+} \\
y_{0}=-\left(A^{-} B^{-}\right)^{+}
\end{gathered}
$$

and such that

$$
\mathcal{B}=\left\{1, A^{-}, B^{-},\left(A^{-} B^{-}\right)^{-}\right\} .
$$

is a basis, then $\rho: G \longrightarrow U_{1}$ such that

$$
\begin{aligned}
& \rho(a)=A=x_{0}+A^{-} \\
& \rho(b)=B=x_{0}+B^{-}
\end{aligned}
$$

is an irreducible c-representation because the entries of the left side of

$$
w(m(A), m(B))\left(\begin{array}{l}
1 \\
0 \\
0 \\
0
\end{array}\right)-\left(\begin{array}{l}
1 \\
0 \\
0 \\
0
\end{array}\right)=\left(\begin{array}{l}
0 \\
0 \\
0 \\
0
\end{array}\right)
$$

are $p_{i}\left(x_{0}, y_{0}\right)=0$ for $i=\{1,2,3,4\}$.

Therefore, to continue with the proof, we will consider the algebra isomorphism

$$
\left(\frac{-1,1}{\mathbb{C}}\right) \longrightarrow M(2, \mathbb{C})
$$

defined by

$$
i \mapsto I=\left(\begin{array}{cc}
0 & 1 \\
-1 & 0
\end{array}\right), \quad j \mapsto J=\left(\begin{array}{cc}
0 & 1 \\
1 & 0
\end{array}\right), \quad i j \mapsto I J=\left(\begin{array}{cc}
1 & 0 \\
0 & -1
\end{array}\right)
$$

sending $U_{1}$ isomorphically onto $S L(2, \mathbb{C})$.

Let $\left(x_{0}, y_{0}\right) \in \mathbb{C} \times \mathbb{C}$ be such that $p_{i}\left(x_{0}, y_{0}\right)=0$ for $i=\{1,2,3,4\}$. A homomorphism $\rho: G \longrightarrow S L(2, \mathbb{C})$ is given by the image of the generators

$$
\rho(a)=A=\left(\begin{array}{ll}
\alpha & \beta \\
\gamma & \delta
\end{array}\right), \quad \rho(b)=B=\left(\begin{array}{ll}
\lambda & \eta \\
\zeta & \xi
\end{array}\right)
$$

Then the condition $A^{+}=B^{+}=x_{0}$ implies that

$$
A^{-}=\left(\begin{array}{cc}
\alpha-x_{0} & \beta \\
\gamma & \delta-x_{0}
\end{array}\right), \quad B^{-}=\left(\begin{array}{cc}
\lambda-x_{0} & \eta \\
\zeta & \xi-x_{0}
\end{array}\right)
$$

The condition $y_{0}=-\left(A^{-} B^{-}\right)^{+}$and the following computation

$$
\begin{aligned}
\left(A^{-} B^{-}\right)^{+} & =\frac{1}{2} \operatorname{tr}\left(A^{-} B^{-}\right)=\frac{1}{2} \operatorname{tr}\left(\left(A-x_{0} I\right)\left(B-x_{0} I\right)\right) \\
& =\frac{1}{2} \operatorname{tr}\left(A B-x_{0}(A+B)+x_{0}^{2} I\right)=\frac{1}{2}\left(\operatorname{tr}(A B)-4 x_{0}^{2}+2 x_{0}^{2}\right) \\
& =\frac{1}{2} \operatorname{tr}(A B)-x_{0}^{2}
\end{aligned}
$$

implies

$$
-y_{0}=\frac{1}{2} \operatorname{tr}(A B)-x_{0}^{2} \quad \Longrightarrow \quad \operatorname{tr}(A B)=2\left(x_{0}^{2}-y_{0}\right)
$$


If $x_{0}^{2} \neq 1$, the elements $A$ and $B$ are not parabolic. Then up to conjugation in $S L(2, \mathbb{C})$ we can assume:

$$
\begin{aligned}
& A=\left(\begin{array}{cc}
x_{0}+\sqrt{x_{0}^{2}-1} & 0 \\
0 & x_{0}-\sqrt{x_{0}^{2}-1}
\end{array}\right) \\
& B=\left(\begin{array}{ll}
\lambda & \eta \\
1 & \xi
\end{array}\right), \quad \lambda+\xi=2 x_{0}, \quad \lambda \xi-\eta=1
\end{aligned}
$$

Therefore

$$
\operatorname{tr}(A B)=2\left(x_{0}^{2}-y_{0}\right)=\left(x_{0}+\sqrt{x_{0}^{2}-1}\right) \lambda+\left(x_{0}-\sqrt{x_{0}^{2}-1}\right) \xi
$$

The three last equations determine unique values for $\lambda, \eta$ and $\xi$, namely:

$$
\lambda=x_{0}-\frac{y_{0}}{\sqrt{x_{0}^{2}-1}} \quad \xi=x_{0}+\frac{y_{0}}{\sqrt{x_{0}^{2}-1}} \quad \eta=\frac{y_{0}^{2}-\left(1-x_{0}^{2}\right)^{2}}{1-x_{0}^{2}}
$$

Under the isomorphism $\left(\frac{-1,1}{\mathbb{C}}\right) \longrightarrow M(2, \mathbb{C})$ we then have:

$$
\begin{aligned}
& A=x_{0}+\sqrt{x_{0}^{2}-1} I J \\
& B=x_{0}+\frac{2-3 x_{0}^{2}+x_{0}^{4}-y_{0}^{2}}{2 x_{0}^{2}-2} I+\frac{-x_{0}^{2}+x_{0}^{4}-y_{0}^{2}}{2 x_{0}^{2}-2} J-\frac{y}{\sqrt{x_{0}^{2}-1}} I J
\end{aligned}
$$

A calculation gives:

$$
\left(A^{-} B^{-}\right)^{-}=\frac{-x_{0}^{2}+x_{0}^{4}-y_{0}^{2}}{2 \sqrt{x_{0}^{2}-1}} I+\frac{2-3 x_{0}^{2}+x_{0}^{4}-y_{0}^{2}}{2 \sqrt{x_{0}^{2}-1}} J
$$

The determinant of the $3 \times 3$ matrix with rows the coordinates of $A^{-}, B^{-},\left(A^{-} B^{-}\right)^{-}$ is, after a tedious calculation:

$$
y_{0}^{2}-\left(1-x_{0}^{2}\right)^{2}
$$

Therefore, by Proposition [7 $\left\{1, A^{-}, B^{-},\left(A^{-} B^{-}\right)^{-}\right\}$is a basis iff $y_{0}^{2} \neq\left(1-x_{0}^{2}\right)^{2}$. Hence if $\left(x_{0}, y_{0}\right)$ belongs to $\left\{(x, y) \in V\left(\mathcal{I}_{G}^{c}\right): y^{2} \neq\left(1-x^{2}\right)^{2}, x^{2} \neq 1\right\}$ there exists an irreducible c-representation $\rho_{\left(x_{0}, y_{0}\right)}: G \longrightarrow U_{1}$, unique up to conjugation in $U_{1}$, realizing $\left(x_{0}, y_{0}\right)$. Namely the one defined by $\rho_{\left(x_{0}, y_{0}\right)}(a)=A, \rho_{\left(x_{0}, y_{0}\right)}(b)=B$.

Now, if $x_{0}^{2} \neq 1$ and $y_{0}^{2}=\left(1-x_{0}^{2}\right)^{2}$ then $\left\{1, A^{-}, B^{-},\left(A^{-} B^{-}\right)^{-}\right\}$is not a basis but the vectors

$$
\begin{aligned}
A^{-} & =\sqrt{x_{0}^{2}-1} I J \\
B^{-} & =-\frac{1}{2} I+\frac{1}{2} J \pm \sqrt{x_{0}^{2}-1} I J
\end{aligned}
$$

are linearly independent because the rank of the $2 \times 3$ matrix with rows the coordinates of $A^{-}, B^{-}$has rank 2 . Then the map $\rho_{\left(x_{0}, y_{0}\right)}: G \longrightarrow U_{1}$, defined by $\rho(a)=A, \rho(b)=B$, is the limit of a sequence of irreducible c-representations $\rho_{\left(x_{i}, y_{i}\right)}$ where $\left\{\left(x_{i}, y_{i}\right)\right\}$ is a sequence of points in the set $V\left(\mathcal{I}_{G}^{c}\right) \backslash V\left(y^{2}-\left(1-x^{2}\right)^{2}\right)$ converging to $\left(x_{0}, y_{0}\right)$. By continuity, $\rho_{\left(x_{0}, y_{0}\right)}: G \longrightarrow U_{1}$ is also a c-representation. It is almost-irreducible, unique up to conjugation in $U_{1}$ and realizes $\left(x_{0}, y_{0}\right)$.

Next we study the case $x_{0}^{2}=1$. There are two cases:

(i) $A \in S L(2, \mathbb{C})$ and $B \in S L(2, \mathbb{C})$ are conjugate parabolic elements with different fixed-points. Then, up to conjugation in $U_{1}$, we can assume

$$
A=\left(\begin{array}{cc}
x_{0} & 1 \\
0 & x_{0}
\end{array}\right), \quad B=\left(\begin{array}{cc}
x_{0} & 0 \\
\lambda & x_{0}
\end{array}\right), \quad \lambda \neq 0
$$

Under the isomorphism $\left(\frac{-1,1}{\mathbb{C}}\right) \longrightarrow M(2, \mathbb{C})$ we have

$$
A^{-}=\frac{1}{2} I+\frac{1}{2} J \quad B^{-}=\frac{\lambda}{2} I+\frac{-\lambda}{2} J \quad A^{-} B^{-}=\frac{\lambda}{2}+\frac{\lambda}{2} I J
$$


and since $y_{0}=-\left(A^{-} B^{-}\right)^{+}$we must have $\lambda=-2 y_{0}$. Therefore $y_{0} \neq 0$ and this implies that $\left\{1, A^{-}, B^{-},\left(A^{-} B^{-}\right)^{-}\right\}$is a basis.

(ii) $A \in S L(2, \mathbb{C})$ and $B \in S L(2, \mathbb{C})$ are conjugate parabolic elements with the same fixed-point or both are

$$
\pm\left(\begin{array}{ll}
1 & 0 \\
0 & 1
\end{array}\right)
$$

Then, up to conjugation in $U_{1}$, we can assume

$$
A=\left(\begin{array}{cc}
x_{0} & \lambda \\
0 & x_{0}
\end{array}\right), B=\left(\begin{array}{cc}
x_{0} & \eta \\
0 & x_{0}
\end{array}\right)
$$

or equivalently

$$
A^{-}=\frac{\lambda}{2} I+\frac{\lambda}{2} J \quad B^{-}=\frac{\eta}{2} I+\frac{\eta}{2} J \quad A^{-} B^{-}=0
$$

Then $y_{0}=-\left(A^{-} B^{-}\right)^{+}=0$ and moreover $A^{-}, B^{-}$are linearly dependent.

Thus if $\left(x_{0}, y_{0}\right) \in V\left(\mathcal{I}_{G}^{c}\right)$, and $x_{0}^{2}=1$, then either $y_{0} \neq 0$ and there is an irreducible c-representation $\rho_{\left(x_{0}, y_{0}\right)}: G \longrightarrow U_{1}$, unique up to conjugation in $U_{1}$, realizing $\left(x_{0}, y_{0}\right)$ (the one defined by $\left.\rho_{\left(x_{0}, y_{0}\right)}(a)=A, \rho_{\left(x_{0}, y_{0}\right)}(b)=B\right)$ or $y_{0}=0$. In this case none of the c-representation $\rho: G \longrightarrow U_{1}$ realizing such $\left(x_{0}, y_{0}\right)$ are irreducible or almost-irreducible. This concludes the proof of the theorem.

Remark 4. The common solutions to $\left\{p_{i}(x, y), i \in\{1,2,3,4\}\right\}$ is a algebraic variety $V\left(\mathcal{I}_{G}^{c}\right)$, the variety of c-representations of $G$ in $U_{1} \cong S L(2, \mathbb{C}) \subset M(2, \mathbb{C})$. If $a$ and $b$ are conjugate elements in $G$, every representation is a c-representation, and the algebraic variety $V\left(\mathcal{I}_{G}^{c}\right)=V\left(\mathcal{I}_{G}\right)$ defines the components of the $S L(2, \mathbb{C})$ character variety of $G$ containing irreducible representations. The $S L(2, \mathbb{C})$-character variety of a group has been already studied in several paper, see for instance, [16], [5], [9], and [13], [14, [12] for a knot group.

Remark 5. In practise, to simplify calculations, it is useful to follow a different approach to compute the polynomials defining the algebraic variety $V\left(\mathcal{I}_{G}^{c}\right)$. The idea is to write the relator of the presentation of $G$ in a balanced way $w_{1}(a, b)=w_{2}(a, b)$ where the words $w_{1}, w_{2}$ have more or less half the length of $w$. Then the condition is

$$
\begin{gathered}
w_{1}(\rho(A), \rho(B))=w_{2}(\rho(A), \rho(B)) \\
\Leftrightarrow \quad w_{1}(\rho(A), \rho(B))-w_{2}(\rho(A), \rho(B))=0
\end{gathered}
$$

In this case the 4 polynomials are obtained by setting

$$
\left(w_{1}(m(A), m(B))-w_{2}(m(A), m(B))\right)\left(\begin{array}{l}
1 \\
0 \\
0 \\
0
\end{array}\right)
$$

This is particularly useful when $G$ is the group of a 2-bridge knot or link.

Example 4. The trefoil knot $3_{1}$.

$$
G=\pi_{1}\left(S^{3}-3_{1}\right)=|a, b: a b a=b a b|
$$

Using this presentation of $G\left(3_{1}\right)$ every representation is a c-representation, because $a$ and $b$ are conjugate elements in $G$. The computation with Mathematica using (4.6) give the polynomials

$$
\left(\begin{array}{c}
0 \\
-1+2 x^{2}-2 y \\
1-2 x^{2}+2 y \\
0
\end{array}\right)
$$


Therefore

$$
p(x, y)=2 x^{2}-2 y-1
$$

defines the ideal $\mathcal{I}_{G}$. If $(x, y)$ corresponds to a almost-irreducible c-representation then $y= \pm\left(1-x^{2}\right)$ and $x^{2} \neq 1$. The solutions are $x=\frac{ \pm \sqrt{3}}{2}, y=\frac{ \pm 1}{4}$. They provide the almost-irreducible c-representations

$$
\rho(a)=\left(\begin{array}{cc}
\frac{ \pm \sqrt{3}+\sqrt{-1}}{2} & 0 \\
0 & \frac{ \pm \sqrt{3}-\sqrt{-1}}{2}
\end{array}\right), \quad \rho(b)=\left(\begin{array}{cc}
\frac{ \pm \sqrt{3}+\sqrt{-1}}{2} & 0 \\
1 & \frac{ \pm \sqrt{3}-\sqrt{-1}}{2}
\end{array}\right) .
$$

But note that besides these almost-irreducible c-representation there might be other reducible c-representations realizing the same values. In fact, for the same point $(x, y)=\left(\frac{\sqrt{3}}{2}, \frac{1}{4}\right)$ the c-representation

$$
\rho(a)=\left(\begin{array}{cc}
\frac{\sqrt{3}+\sqrt{-1}}{2} & 0 \\
0 & \frac{\sqrt{3}-\sqrt{-1}}{2}
\end{array}\right), \quad \rho(b)=\left(\begin{array}{cc}
\frac{\sqrt{3}+\sqrt{-1}}{2} & 0 \\
0 & \frac{\sqrt{3}-\sqrt{-1}}{2}
\end{array}\right)
$$

is reducible (not almost-reducible).

Remark 6. If

$$
\begin{aligned}
\rho(a) & =A=x_{0}+A^{-} \\
\rho(b) & =B=x_{0}+B^{-} \\
y_{0} & =-\left(A^{-} B^{-}\right)^{+}
\end{aligned}
$$

then $\operatorname{tr}(A B)=-2 y_{0}+2 x_{0}^{2}$. This gives us the formulas to write the algebraic variety $V\left(\mathcal{I}_{G}^{c}\right)$ in terms of the variables $\operatorname{tr}\left(A^{2}\right)$ and $\operatorname{tr}(A B)$ as in the papers [13] and [14]. Lets call $x^{\prime}$ the variable $x$ used in those papers to avoid mistakes. The variables in 13 and 14 are

$$
x^{\prime}=\operatorname{tr}\left(A^{2}\right)=(\operatorname{tr}(A))^{2}-2 \quad \text { and } \quad z=\operatorname{tr}(A B)
$$

Therefore the change from $\left(x^{\prime}, z\right)$ to our variables $(x, y)$ in the present work is

$$
\begin{aligned}
x^{\prime} & =4 x^{2}-2 \\
z & =2 x^{2}-2 y
\end{aligned}
$$

In the above example, the polynomial

$$
p(x, y)=2 x^{2}-2 y-1
$$

in the variables $x^{\prime}$ and $z$ is $p\left(x^{\prime}, z\right)=z-1$.

The above Theorem 3 can be sharpened in case $x_{0}, y_{0} \in \mathbb{R}$ :

Theorem 4. Let $G$ be a group given by the presentation

$$
G=|a, b: w(a, b)|
$$

where $w$ is a word in a and $b$. Let $\mathcal{I}_{G}^{c}$ be the ideal generated by the four polynomials

$$
\left\{p_{1}(x, y), p_{2}(x, y), p_{3}(x, y), p_{4}(x, y)\right\}
$$

with integer coefficients. If $\left(x_{0}, y_{0}\right)$ is a real point of the algebraic variety $V\left(\mathcal{I}_{G}^{c}\right)$ we distinguish two cases:

(1) If

$$
\begin{gathered}
1-x_{0}^{2}>0, \\
\left(1-x_{0}^{2}\right)^{2}>y_{0}^{2}
\end{gathered}
$$


there exists an irreducible c-representation $\rho: G \longrightarrow U_{1}, U_{1}=S^{3} \subset$ $\mathbb{H}$, unique up to conjugation in $U_{1}=S^{3}$, realizing $\left(x_{0}, y_{0}\right)$. Namely:

$$
\begin{aligned}
& A=x_{0}+\frac{1}{+\sqrt{1-x_{0}^{2}}}\left(y_{0} i+\sqrt{\left(1-x_{0}^{2}\right)^{2}-y_{0}^{2}} j\right) \\
& B=x_{0}+\sqrt{1-x_{0}^{2}} i, \quad \sqrt{1-x_{0}^{2}}>0
\end{aligned}
$$

$$
\left(A^{-} B^{-}\right)^{-}=-\sqrt{\left(1-x^{2}\right)^{2}-y^{2}} i j
$$

and $\rho(G) \subset\left(\frac{-1,-1}{k}\right)$ where

$$
k=Q\left(x_{0}, y_{0}, \sqrt{1-x_{0}^{2}}, \sqrt{\left(1-x_{0}^{2}\right)^{2}-y_{0}^{2}}\right) .
$$

(2) The remaining cases. Then excepting the case

$$
\begin{gathered}
1-x_{0}^{2}>0, \\
\left(1-x_{0}^{2}\right)^{2}=y_{0}^{2}
\end{gathered}
$$

and the case

$$
x_{0}^{2}=1, y_{0}=0
$$

there exists an irreducible (or almost-irreducible) c-representation $\rho: G \longrightarrow$ $U_{1}, U_{1}=S L(2, \mathbb{R}) \subset\left(\frac{-1,1}{\mathbb{R}}\right)$ realizing $\left(x_{0}, y_{0}\right)$. Moreover two such homomorphisms are equal up to conjugation in $U_{ \pm 1}$. Specifically:

(2.1) If

$$
\begin{gathered}
1-x_{0}^{2}>0, \\
\left(1-x_{0}^{2}\right)^{2}<y_{0}^{2}
\end{gathered}
$$

set

$$
\begin{gathered}
A=x_{0}+\sqrt{1-x_{0}^{2}} I, \quad \sqrt{1-x_{0}^{2}}>0 \\
B=x_{0}+\frac{1}{+\sqrt{1-x_{0}^{2}}}\left(y_{0} I+\sqrt{y_{0}^{2}-\left(1-x_{0}^{2}\right)^{2}} J\right)
\end{gathered}
$$

Then $\rho: G \longrightarrow U_{1}$ is irreducible, $\rho(G) \subset\left(\frac{-1,1}{k}\right)$ where

$$
k=Q\left(x_{0}, y_{0}, \sqrt{1-x_{0}^{2}}, \sqrt{y_{0}^{2}-\left(1-x_{0}^{2}\right)^{2}}\right)
$$

and

$$
\left(A^{-} B^{-}\right)^{-}=+\sqrt{y_{0}^{2}-\left(1-x_{0}^{2}\right)^{2}} I J
$$

(2.2) If

$$
\begin{gathered}
1-x_{0}^{2}<0, \\
\left(1-x_{0}^{2}\right)^{2}<y_{0}^{2}
\end{gathered}
$$

there are two subcases:

(2.2.1) $y_{0}<0$. Set

$$
\begin{gathered}
A=x_{0}+\sqrt{x_{0}^{2}-1} J, \quad \sqrt{x_{0}^{2}-1}>0 \\
B=x_{0}+\frac{1}{+\sqrt{x_{0}^{2}-1}}\left(\sqrt{y_{0}^{2}-\left(x_{0}^{2}-1\right)^{2}} I-y_{0} J\right)
\end{gathered}
$$

Then $\rho: G \longrightarrow U_{1}$ is irreducible and

$$
\left(A^{-} B^{-}\right)^{-}=-\sqrt{y_{0}^{2}-\left(x_{0}^{2}-1\right)^{2}} I J
$$

(2.2.2) $y_{0}>0$. Set

$$
\begin{gathered}
A=x_{0}+\sqrt{x_{0}^{2}-1} J, \quad \sqrt{x_{0}^{2}-1}>0 \\
B=x_{0}-\frac{1}{+\sqrt{x_{0}^{2}-1}}\left(\sqrt{y_{0}^{2}-\left(x_{0}^{2}-1\right)^{2}} I+y_{0} J\right)
\end{gathered}
$$


Then $\rho: G \longrightarrow U_{1}$ is irreducible and

$$
\left(A^{-} B^{-}\right)^{-}=+\sqrt{y_{0}^{2}-\left(x_{0}^{2}-1\right)^{2}} I J
$$

In both cases $\rho(G) \subset\left(\frac{-1,1}{k}\right)$ where

$$
k=Q\left(x_{0}, y_{0}, \sqrt{x_{0}^{2}-1}, \sqrt{y_{0}^{2}-\left(x_{0}^{2}-1\right)^{2}}\right) .
$$

(2.3) If

$$
\begin{gathered}
1-x_{0}^{2}<0 \\
\left(1-x_{0}^{2}\right)^{2}>y_{0}^{2}
\end{gathered}
$$

set

$$
\begin{gathered}
A=x_{0}+\sqrt{x_{0}^{2}-1} J, \quad \sqrt{x_{0}^{2}-1}>0 \\
B=x_{0}+\frac{1}{+\sqrt{x_{0}^{2}-1}}\left(-y_{0} J+\sqrt{\left(x_{0}^{2}-1\right)^{2}-y_{0}^{2}} I J\right)
\end{gathered}
$$

Then $\rho: G \longrightarrow U_{1}$ is irreducible, $\rho(G) \subset\left(\frac{-1,1}{k}\right)$ where

$$
k=Q\left(x_{0}, y_{0}, \sqrt{x_{0}^{2}-1}, \sqrt{\left(x_{0}^{2}-1\right)^{2}-y_{0}^{2}}\right)
$$

and

$$
\left(A^{-} B^{-}\right)^{-}=-\sqrt{\left(x_{0}^{2}-1\right)^{2}-y_{0}^{2}} I J
$$

(2.4) If

$$
\begin{gathered}
1-x_{0}^{2}<0 \\
\left(1-x_{0}^{2}\right)^{2}=y_{0}^{2}
\end{gathered}
$$

set

$$
\begin{gathered}
A=x_{0}+\sqrt{x_{0}^{2}-1} J, \quad \sqrt{x_{0}^{2}-1}>0 \\
B=x_{0}+(I J+I)-\frac{y_{0}}{\sqrt{x_{0}^{2}-1}} J
\end{gathered}
$$

Then $\rho: G \longrightarrow U_{1}$ is almost-irreducible, $\rho(G) \subset\left(\frac{-1,1}{k}\right)$ where

$$
k=Q\left(x_{0}, y_{0}, \sqrt{x_{0}^{2}-1}\right)
$$

and

(2.5) If

$$
\left(A^{-} B^{-}\right)^{-}=-\sqrt{\left(x_{0}^{2}-1\right)}(I+I J)
$$

$$
\begin{gathered}
1-x_{0}^{2}=0, \\
y_{0} \neq 0
\end{gathered}
$$

set

$$
\begin{gathered}
A=x_{0}+I+J \\
B=x_{0}+\frac{y_{0}}{2}(I-J) \\
\left(A^{-} B^{-}\right)^{-}=-y_{0} I J
\end{gathered}
$$

Then $\rho: G \longrightarrow U_{1}$ is irreducible and $\rho(G) \subset\left(\frac{-1,1}{k}\right)$ where $k=Q\left(y_{0}\right)$.

Proof. As in the proof of the Theorem 3 we only need to find quaternions $A$ and $B$ in the appropriate $U_{1}$ such that

$$
\begin{gathered}
x_{0}=A^{+}=B^{+} \\
y_{0}=-\left(A^{-} B^{-}\right)^{+}
\end{gathered}
$$

and such that either $\left\{1, A^{-}, B^{-},\left(A^{-} B^{-}\right)^{-}\right\}$is a basis (case irreducible) or that $A^{-}$ and $B^{-}$are linearly independent (case almost-irreducible), because then $\rho: G \longrightarrow$ $U_{1}$ such that

$$
\begin{aligned}
& \rho(a)=A=x_{0}+A^{-} \\
& \rho(b)=B=x_{0}+B^{-}
\end{aligned}
$$


will be the desired homomorphism.

In case 1 , the desired quaternions (unique, up to conjugation in $U_{1}$ ) are provided by Theorem 1 .

In case 2 apply Theorem 2 ,

Remark 7. To apply Theorem 4 it is useful to consider the pattern of Figure 15. It shows the real plane with coordinates $x$ and $\frac{y}{1-x^{2}}$. The plane is divided in several labeled regions by labeled segments. The label corresponds to the case described in Theorem 4. Therefore, to apply Theorem 4 to the algebraic variety $V\left(\mathcal{I}_{G}\right)$ it is enough to study the graphic of $\frac{y}{1-x^{2}}$ as a funtion of $x$ over the pattern.

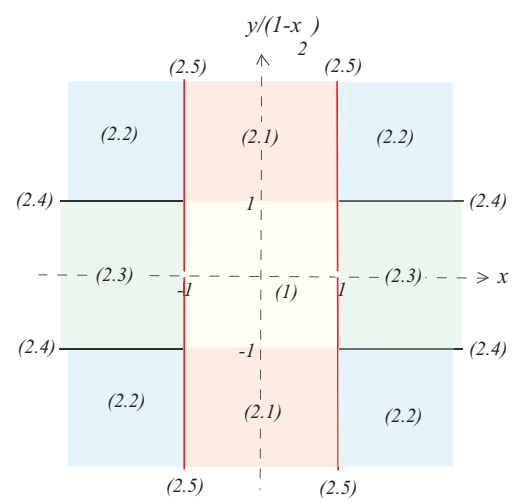

Figure 15. The pattern for real points.

The following remark concerns the points in the unlabeled segments in the pattern between region (1) and region (2.1).

Remark 8. In the case that $\left(x_{0}, y_{0}\right)$ is a real point of the algebraic variety $V\left(\mathcal{I}_{G}^{c}\right)$ and

$$
\begin{gathered}
1-x_{0}^{2}>0, \\
\left(1-x_{0}^{2}\right)^{2}=y_{0}^{2}
\end{gathered}
$$

then, according to Theorem 3 there is an almost-irreducible c-representation $\rho$ : $G \longrightarrow S L(2, \mathbb{C})$ realizing $\left(x_{0}, y_{0}\right)$ but no almost-irreducible c-representations $\rho$ : $G \longrightarrow S L(2, \mathbb{R})$ or $\rho: G \longrightarrow S^{3}$ realizing $\left(x_{0}, y_{0}\right)$ exists, because the cases $(1)$ and (2.1) in Theorem 4 both contain the limiting case

$$
\begin{gathered}
1-x_{0}^{2}>0, \\
\left(1-x_{0}^{2}\right)^{2}=y_{0}^{2}
\end{gathered}
$$

and then $A^{-}$and $B^{-}$coincide, so that $\rho$ is not almost-irreducible. An example is given above, where the almost-irreducible c-representation $\rho: \pi_{1}\left(S^{3}-3_{1}\right) \rightarrow$ $S L(2, \mathbb{C})$ granted by Theorem 3 and corresponding to $x_{0}=\frac{\sqrt{3}}{2}, y_{0}=\frac{1}{4}$ is

$$
\rho(a)=\left(\begin{array}{cc}
\frac{\sqrt{3}+\sqrt{-1}}{2} & 0 \\
0 & \frac{\sqrt{3}-\sqrt{-1}}{2}
\end{array}\right), \quad \rho(b)=\left(\begin{array}{cc}
\frac{\sqrt{3}+\sqrt{-1}}{2} & 0 \\
1 & \frac{\sqrt{3}-\sqrt{-1}}{2}
\end{array}\right)
$$

However, since $1-x_{0}^{2}=\frac{1}{4}>0$ and $\left(1-x_{0}^{2}\right)^{2}=\frac{1}{16}=y_{0}^{2}$ almost-irreducible crepresentations $\rho: \pi_{1}\left(S^{3}-3_{1}\right) \longrightarrow S L(2, \mathbb{R})$ or $\rho: \pi_{1}\left(S^{3}-3_{1}\right) \longrightarrow S^{3}$ realizing $\left(x_{0}, y_{0}\right)=\left(\frac{\sqrt{3}}{2}, \frac{1}{4}\right)$ do not exist. This is in striking contrast with the case

$$
\begin{gathered}
1-x_{0}^{2}<0, \\
\left(1-x_{0}^{2}\right)^{2}=y_{0}^{2}
\end{gathered}
$$


$\left(\left(x_{0}, y_{0}\right)\right.$ a real point) in which an almost-irreducible c-representation $\rho: G \longrightarrow$ $S L(2, \mathbb{R})$ realizing $\left(x_{0}, y_{0}\right)$ does in fact exist (case (2.4) of Theorem 4 ). An example follows.

Example 5. The figure-eight knot $4_{1}$.

$$
G\left(4_{1}\right)=\pi_{1}\left(S^{3}-4_{1}\right)=|a, b: a w=w b|, \quad w=b a^{-1} b^{-1} a
$$

Using this presentation of $G\left(4_{1}\right)$ every representation is a c-representation, because $a$ and $b$ are conjugate elements in $G\left(4_{1}\right)$. The computation with Mathematica gives the following polynomials defining $\mathcal{I}_{G\left(4_{1}\right)}$ :

$$
\left(\begin{array}{c}
0 \\
1-6 x^{2}+4 x^{4}-2 y-4 y^{2} \\
1-6 x^{2}+4 x^{4}-2 y-4 y^{2} \\
0
\end{array}\right)
$$

Therefore

$$
p(x, y)=1-6 x^{2}+4 x^{4}-2 y-4 y^{2}
$$

defines the ideal $\mathcal{I}_{G\left(4_{1}\right)}$. The points $\left(x_{0}, y_{0}\right) \in V\left(\mathcal{I}_{G\left(4_{1}\right)}\right)$ with $y_{0}= \pm\left(1-x_{0}^{2}\right)$ are $\left( \pm \frac{\sqrt{5}}{2},-\frac{1}{4}\right)$. Note that $1-x_{0}^{2}=-\frac{1}{4}<0$. Then, case (2.4) of Theorem 4 applies and there exists the following almost-irreducible c-representation (see [9])

$$
\rho: G\left(4_{1}\right) \longrightarrow U_{1} \subset\left(\frac{-1,1}{\mathbb{R}}\right)
$$

realizing $\left(x_{0}, y_{0}\right)=\left(\frac{\sqrt{5}}{2},-\frac{1}{4}\right)$ :

$$
\begin{gathered}
\rho(a)=A=\frac{\sqrt{5}}{2}+\frac{1}{2} J, \\
\rho(b)=B=\frac{\sqrt{5}}{2}+I+\frac{1}{2} J+I J
\end{gathered}
$$

and after identifying $\left(\frac{-1,1}{\mathbb{R}}\right)$ with $M(2, \mathbb{R})$ via

$$
a+b I+c J+d I J \longleftrightarrow\left(\begin{array}{cc}
a+d & b+c \\
-b+c & a-d
\end{array}\right)
$$

we have $\rho: \pi_{1}\left(S^{3}-4_{1}\right) \longrightarrow S L(2, \mathbb{R})$ :

$$
\begin{gathered}
\rho(a)=A=\left(\begin{array}{cc}
1+\frac{\sqrt{5}}{2} & \frac{3}{2} \\
-\frac{1}{2} & -1+\frac{\sqrt{5}}{2}
\end{array}\right) \\
\rho(b)=B=\left(\begin{array}{cc}
\frac{\sqrt{5}}{2} & \frac{1}{2} \\
\frac{1}{2} & \frac{\sqrt{5}}{2}
\end{array}\right)
\end{gathered}
$$

4.1. Reducible c-representations. As we saw before if the c-representation $\rho$ : $G \longrightarrow U_{1}$ is almost-irreducible then $y^{2}=\left(1-x^{2}\right)^{2}$. If $\rho$ is reducible and $B^{-}=$ $\pm A^{-}$this implies also $y^{2}=\left(1-x^{2}\right)^{2}$. The algebraic variety $V\left(y^{2}-\left(1-x^{2}\right)^{2}\right)$ is the union of two parabolas of equations $y=1-x^{2}$ and $y=x^{2}-1$. Given a point $\left(x_{0}, y_{0}\right) \in V\left(y^{2}-\left(1-x^{2}\right)^{2}\right)$ we want to know if there exists a reducible c-representation $\rho: G \longrightarrow U_{1}$ realizing it.

Case 1. $\left(x_{0}, y_{0}\right) \in V\left(y+x^{2}-1\right)$. The putative reducible c-representation realizing $\left(x_{0}, y_{0}\right)$ must be defined by

$$
\rho(a)=x+A^{-}, \quad \rho(b)=x+B^{-}, \quad A^{-}=B^{-}
$$

because then

$$
y=-\left(A^{-} B^{-}\right)^{+}=-\left(A^{-} A^{-}\right)^{+}=-A^{-} A^{-}=1-x^{2}
$$


since

$$
1=N(A)=A \bar{A}=\left(x+A^{-}\right)\left(x-A^{-}\right)=x^{2}-A^{-} A^{-}
$$

A necessary and sufficient condition for the existence of such a c-representation

$$
\rho: G=|a, b: w(a, b)| \longrightarrow U_{1}
$$

is

$$
w\left(x+A^{-}, x+A^{-}\right)=1
$$

A sufficient condition for this is that the group presented by:

$$
|a, b: w(a, b)=1 ; a=b|
$$

be trivial. This happens for the standard presentations of the groups of 2-bridge knots and links $(|a, b: a v(a, b)=v(a, b) b|$ for 2-bridge knots and $|a, b: a v(a, b)=v(a, b) a|$ for 2-bridge links).

We conclude that:

Proposition 9. The points of the algebraic variety $V\left(y+x^{2}-1\right)$ can be realized by reducible c-representations $\rho: G \longrightarrow U_{1}$ if $G$ is the group of a 2-bridge knot or link.

Case 2. $\left(x_{0}, y_{0}\right) \in V\left(y-x^{2}+1\right)$. The putative reducible c-representation realizing $\left(x_{0}, y_{0}\right)$ must be defined by

$$
\rho(a)=x+A^{-}, \quad \rho(b)=x+B^{-}, \quad B^{-}=-A^{-}
$$

because then

$$
y=-\left(A^{-} B^{-}\right)^{+}=\left(A^{-} A^{-}\right)^{+}=A^{-} A^{-}=-1+x^{2}
$$

since

$$
1=N(A)=A \bar{A}=\left(x+A^{-}\right)\left(x-A^{-}\right)=x^{2}-A^{-} A^{-}
$$

A necessary and sufficient condition for the existence of such a c-representation

$$
\rho: G=|a, b: w(a, b)| \longrightarrow U_{1}
$$

is

$$
w\left(x+A^{-}, x-A^{-}\right)=1
$$

A sufficient condition for this is that the group presented by:

$$
\left|a, b: w(a, b)=1 ; b=a^{-1}\right|
$$

be trivial. This happens for the standard presentations $|a, b: a v(a, b)=v(a, b) a|$ of the group of a 2-bridge link.

For the standard presentations $|a, b: a v(a, b)=v(a, b) b|$ of the group of a 2-bridge knot, the relation

$$
\left(x+A^{-}\right) v\left(x+A^{-}, x-A^{-}\right)=v\left(x+A^{-}, x-A^{-}\right)\left(x-A^{-}\right)
$$

implies either

$$
\left(x+A^{-}\right)\left(x+A^{-}\right)^{n}=\left(x+A^{-}\right)^{n}\left(x-A^{-}\right), \quad n \geq 0
$$

or

$$
\left(x+A^{-}\right)\left(x-A^{-}\right)^{n}=\left(x-A^{-}\right)^{n}\left(x-A^{-}\right), \quad n \geq 1
$$

because $1=N(A)=A \bar{A}=\left(x+A^{-}\right)\left(x-A^{-}\right)$. Both cases imply $\left(x+A^{-}\right)=$ $\left(x-A^{-}\right)$. That is $A^{-}=0$, which corresponds to the point $\left(x_{0}, y_{0}\right)=( \pm 1,0)$.

We conclude that:

Proposition 10. The points of the algebraic variety $V\left(y-x^{2}+1\right)$ can be realized by reducible c-representations $\rho: G \longrightarrow U_{1}$ if $G$ is the group of a 2-bridge link but not if $G$ is the group of a 2-bridge knot. 
4.2. Two examples. Next we analyze the real points of the algebraic variety $V\left(\mathcal{I}_{G}\right)$ for two knot groups.

4.2.1. The Trefoil knot. As was said in Example 4 the algebraic variety $V\left(\mathcal{I}_{G\left(3_{1}\right)}\right)$ for the Trefoil knot group

$$
G(3 / 1)=|a, b ; a b a=b a b|
$$

is define by the ideal $\mathcal{I}_{G\left(3_{1}\right)}=\left(2 x^{2}-2 y-1\right)$. The real part of $V\left(\mathcal{I}_{G\left(3_{1}\right)}\right)$ is the parabola $y=\frac{2 x^{2}-1}{2}$ depicted in Figure 16.

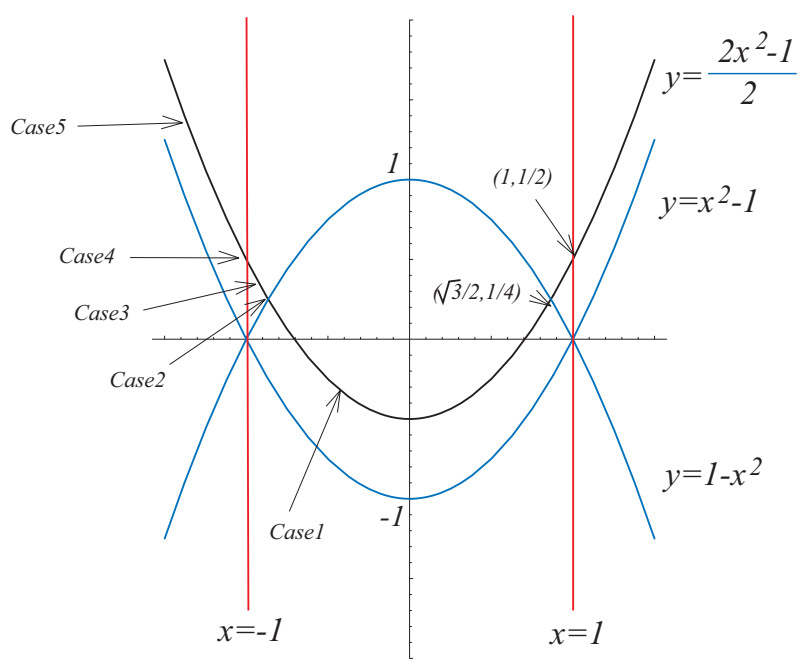

Figure 16. The real curve $V\left(\mathcal{I}_{G\left(3_{1}\right)}\right)$ and the five cases.

Figure 16] also exhibits the variety of reducible representations $V\left(y^{2}-\left(x^{2}-1\right)^{2}\right)$. This is the union of the two parabolas $y= \pm\left(x^{2}-1\right)$. The trefoil knot is a 2-bridge knot. Then, by Propositions 9 and 10, only the points of the parabola $y=1-x^{2}$ can be realized by reducible c-representations. The intersection of $V\left(\mathcal{I}_{G\left(3_{1}\right)}\right)$ with $V\left(y-1+x^{2}\right)$ define the two almost-irreducible representations realizing the pairs $(x, y)=\left( \pm \frac{\sqrt{3}}{2}, \frac{1}{4}\right)$ described in Example 4. The remaining points of $V\left(\mathcal{I}_{G\left(3_{1}\right)}\right)$ correspond to irreducible c-representations.

Theorem 4 establishes the different cases of representations associated to real points of the algebraic variety $V\left(\mathcal{I}_{G\left(3_{1}\right)}\right)$. To apply Theorem 4 to the algebraic variety $V\left(\mathcal{I}_{G}\right)$ it suffices to study the graphic of $\frac{y}{1-x^{2}}$ as a function of $x$ over the pattern of Remark 7. See Figure 15.

Figure 17 shows $\frac{y}{1-x^{2}}$ as a function of $x$ for the algebraic variety $V\left(\mathcal{I}_{G\left(3_{1}\right)}\right)$.

Then, according with Theorem 4 , there are five cases:

Case 1: Region (1). $x \in\left(\frac{-\sqrt{3}}{2}, \frac{\sqrt{3}}{2}\right) \Longleftrightarrow\left\{1-x^{2}>0,\left(1-x^{2}\right)^{2}>y^{2}\right\}$, where

$$
\begin{aligned}
& y=\frac{2 x^{2}-1}{2} \\
& u=1-x^{2}
\end{aligned}
$$

There exists an irreducible c-representation $\rho_{x}: G(3 / 1) \longrightarrow S^{3}$ realizing $(x, y)$, unique up to conjugation in $S^{3}$, such that

$$
\begin{aligned}
& \rho_{x}(a)=A=x+\frac{2 x^{2}-1}{2 \sqrt{1-x^{2}}} i+\frac{1}{2} \sqrt{\frac{3-4 x^{2}}{1-x^{2}}} j \\
& \rho_{x}(b)=B=x+\sqrt{1-x^{2}} i, \quad \sqrt{1-x^{2}}>0
\end{aligned}
$$




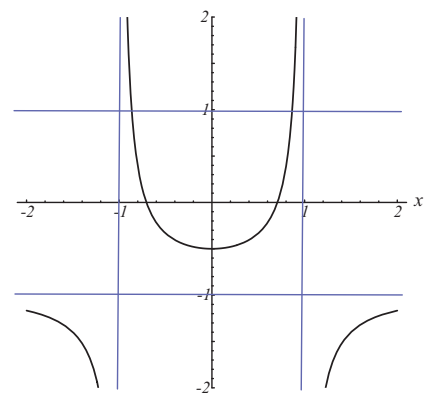

Figure 17. The function $\frac{y}{1-x^{2}}$ for the Trefoil knot.

The composition of $\rho_{x}$ with $c: S^{3} \rightarrow S O(3)$, where $c(X), X \in S^{3}$, acts on $P \in H_{0} \cong E^{3}$ by conjugation, defines the representation $\rho_{x}^{\prime}=c \circ \rho_{x}$ : $G(3 / 1) \longrightarrow S O(3)$. In linear notation, where $\{X, Y, Z\}$ is the coordinate system in $E^{3}$ associated to the basis $\{-i j, j, i\}$ we have

$$
\begin{aligned}
& \rho_{x}^{\prime}(a)=m_{x}(a)\left(\begin{array}{c}
X \\
Y \\
Z
\end{array}\right)=\left(\begin{array}{c}
X^{\prime} \\
Y^{\prime} \\
Z^{\prime}
\end{array}\right) \\
& \rho_{x}^{\prime}(b)=m_{x}(b)\left(\begin{array}{c}
X \\
Y \\
Z
\end{array}\right)=\left(\begin{array}{c}
X^{\prime} \\
Y^{\prime} \\
Z^{\prime}
\end{array}\right)
\end{aligned}
$$

where, by (2.3),

$$
m_{x}(a)=\left(\begin{array}{ccc}
2 x^{2}-1 & \frac{x-2 x^{3}}{\sqrt{1-x^{2}}} & x \sqrt{\frac{4 x^{2}-3}{x^{2}-1}} \\
\frac{x\left(2 x^{2}-1\right)}{\sqrt{1-x^{2}}} & \frac{-4 x^{4}+2 x^{2}+1}{2-2 x^{2}} & \frac{\left(1-2 x^{2}\right) \sqrt{3-4 x^{2}}}{2\left(x^{2}-1\right)} \\
-x \sqrt{\frac{4 x^{2}-3}{x^{2}-1}} & \frac{\left(1-2 x^{2}\right) \sqrt{3-4 x^{2}}}{2\left(x^{2}-1\right)} & \frac{1-2 x^{2}}{2 x^{2}-2}
\end{array}\right)
$$

and

$$
m_{x}(b)=\left(\begin{array}{ccc}
2 x^{2}-1 & -2 x \sqrt{1-x^{2}} & 0 \\
2 x \sqrt{1-x^{2}} & 2 x^{2}-1 & 0 \\
0 & 0 & 1
\end{array}\right) .
$$

The maps $\rho_{x}^{\prime}(a)$ and $\rho_{x}^{\prime}(b)$ are right rotations of angle $\alpha$ around the axes $A^{-}$and $B^{-}$where $x=A^{+}=B^{+}=\cos \frac{\alpha}{2}$. See Figure 6. The angle $\omega$ between the axes of $\rho_{x}^{\prime}(a)$ and $\rho_{x}^{\prime}(b)$ is given by

$$
\cos \omega=\frac{y}{u}=\frac{x^{2}-\frac{1}{2}}{1-x^{2}}
$$

Case 2: $(x, y)=\left( \pm \frac{\sqrt{3}}{2}, \frac{1}{4}\right) \Longleftrightarrow\left\{1-x^{2}>0,\left(1-x^{2}\right)^{2}=y^{2}\right\}$.

There exists an almost-irreducible c-representation $\rho_{x}: G(3 / 1) \longrightarrow U_{1} \subset$ $\left(\frac{-1,1}{\mathbb{C}}\right)$ realizing $(x, y)$, unique up to conjugation in $U_{1}$ (Theorem 3 , (4.5) ), such that:

$$
\begin{aligned}
& \rho_{ \pm \sqrt{3} / 2}(a)=A=\frac{ \pm \sqrt{3}}{2}+\frac{\sqrt{-1}}{2} I J \\
& \rho_{ \pm \sqrt{3} / 2}(b)=B=\frac{ \pm \sqrt{3}}{2}-\frac{1}{2} I+\frac{1}{2} J+\frac{\sqrt{-1}}{2} I J
\end{aligned}
$$


This representation cannot be conjugated to any real representation. Under the isomorphism $U_{1} \approx S L(2, \mathbb{C})$ we have

$$
\begin{aligned}
& \rho_{ \pm \sqrt{3} / 2}: G(3 / 1) \quad \longrightarrow \quad S L(2, \mathbb{C}) \\
& a \quad \rightarrow \quad A=\left(\begin{array}{cc}
\frac{ \pm \sqrt{3}}{2}+\frac{\sqrt{-1}}{2} & 0 \\
0 & \frac{ \pm \sqrt{3}}{2}-\frac{\sqrt{-1}}{2}
\end{array}\right) \\
& b \quad \rightarrow \quad B=\left(\begin{array}{cc}
\frac{ \pm \sqrt{3}}{2}+\frac{\sqrt{-1}}{2} & 0 \\
1 & \frac{ \pm \sqrt{3}}{2}-\frac{\sqrt{-1}}{2}
\end{array}\right)
\end{aligned}
$$

Case 3: Region (2.1). $x \in\left(-1, \frac{-\sqrt{3}}{2}\right) \cup\left(\frac{\sqrt{3}}{2}, 1\right) \Longleftrightarrow\left\{1-x^{2}>0,\left(1-x^{2}\right)^{2}<y^{2}\right\}$.

There exists an irreducible c-representation $\rho_{x}: G(3 / 1) \longrightarrow S L(2, \mathbb{R})=$ $U_{1} \subset\left(\frac{-1,1}{\mathbb{R}}\right)$ realizing $(x, y)$, unique up to conjugation in $S L(2, \mathbb{R})$, such that

$$
\begin{aligned}
& \rho_{x}(a)=A=x+\sqrt{1-x^{2}} I, \quad \sqrt{1-x^{2}}>0 \\
& \rho_{x}(b)=B=x+\frac{2 x^{2}-1}{2 \sqrt{1-x^{2}}} I+\frac{1}{2} \sqrt{\frac{-3+4 x^{2}}{1-x^{2}}} J
\end{aligned}
$$

The composition of $\rho_{x}$ with $c: S L(2, \mathbb{R}) \rightarrow S O^{+}(1,2) \cong I s O^{+}\left(\mathbb{H}^{2}\right)$, where $c(X), X \in S L(2, \mathbb{R})$, acts on $P \in H_{0} \cong E^{1,2}$ by conjugation, defines the representation $\rho_{x}^{\prime}=c \circ \rho_{x}: G(3 / 1) \longrightarrow S O^{+}(1,2)$ in affine linear notation, where $\{X, Y, Z\}$ is the coordinate system associated to the basis $\{-I J, J, I\}$

$$
\begin{aligned}
& \rho_{x}^{\prime}(a)=m_{x}(a)\left(\begin{array}{c}
X \\
Y \\
Z
\end{array}\right)=\left(\begin{array}{c}
X^{\prime} \\
Y^{\prime} \\
Z^{\prime}
\end{array}\right) \\
& \rho_{x}^{\prime}(b)=m_{x}(b)\left(\begin{array}{c}
X \\
Y \\
Z
\end{array}\right)=\left(\begin{array}{c}
X^{\prime} \\
Y^{\prime} \\
Z^{\prime}
\end{array}\right)
\end{aligned}
$$

where the matrices of $\rho_{x}^{\prime}(a)$ and $\rho_{x}^{\prime}(b)$ are respectively:

$$
m_{x}(a)=\left(\begin{array}{ccc}
2 x^{2}-1 & -2 x \sqrt{1-x^{2}} & 0 \\
2 x \sqrt{1-x^{2}} & 2 x^{2}-1 & 0 \\
0 & 0 & 1
\end{array}\right)
$$

and

$$
m_{x}(b)=\left(\begin{array}{ccc}
2 x^{2}-1 & \frac{x-2 x^{3}}{\sqrt{1-x^{2}}} & x \sqrt{\frac{3-4 x^{2}}{x^{2}-1}} \\
\frac{x\left(2 x^{2}-1\right)}{\sqrt{1-x^{2}}} & \frac{1+2 x^{2}-4 x^{4}}{2-2 x^{2}} & \frac{\sqrt{4 x^{2}-3}\left(2 x^{2}-1\right)}{2\left(1-x^{2}\right)} \\
x \sqrt{\frac{3-4 x^{2}}{x^{2}-1}} & \frac{\sqrt{4 x^{2}-3}\left(2 x^{2}-1\right)}{2\left(1-x^{2}\right)} & \frac{1-2 x^{2}}{2 x^{2}-2}
\end{array}\right)
$$

The maps $\rho_{x}^{\prime}(a)$ and $\rho_{x}^{\prime}(b)$ are right (spherical) rotations of $H_{0} \cong E^{1,2}$ of angle $\alpha$ around the time-like axes $A^{-}$and $B^{-}$where $x=A^{+}=B^{+}=\cos \frac{\alpha}{2}$. See Figure 7.

The distance $d$ (measured in the hyperbolic plane) between the axes of $\rho_{x}^{\prime}(a)$ and $\rho_{x}^{\prime}(b)$ is given by

$$
\cosh d=\frac{y}{u}=\frac{x^{2}-\frac{1}{2}}{-x^{2}+1}
$$

Case 4: Segment (2.5). $(x, y)=\left( \pm 1, \frac{1}{2}\right) \Longleftrightarrow\left\{1-x^{2}=0,\left(1-x^{2}\right)^{2}<y^{2}\right\}$

There exists an irreducible c-representation $\rho_{x}: G(3 / 1) \longrightarrow S L(2, \mathbb{R})=$ $U_{1} \subset\left(\frac{-1,1}{\mathbb{R}}\right)$ realizing $(x, y)$, unique up to conjugation in $S L(2, \mathbb{R})$, such that

$$
\begin{aligned}
& \rho_{x}(a)=A= \pm 1+I+J \\
& \rho_{x}(b)=B= \pm 1+\frac{1}{4}(I-J)
\end{aligned}
$$


The composition of $\rho_{x}$ with $c: S L(2, \mathbb{R}) \rightarrow S O^{+}(1,2) \cong I s 0^{+}\left(\mathbb{H}^{2}\right)$, defines the representation $\rho_{x}^{\prime}=c \circ \rho_{x}: G(3 / 1) \longrightarrow S O^{+}(1,2)$ such that the matrices of $\rho_{x}^{\prime}(a)$ and $\rho_{x}^{\prime}(b)$ are respectively:

$$
m_{x}(a)=m(-1,1 ; \pm 1,1,1,0)=\left(\begin{array}{ccc}
1 & -2 & 2 \\
2 & -1 & 2 \\
2 & -2 & 3
\end{array}\right)
$$

and

$$
m_{x}(b)=m\left(-1,1 ; \pm 1, \frac{1}{4},-\frac{1}{4}, 0\right)=\left(\begin{array}{ccc}
1 & -\frac{1}{2} & -\frac{1}{2} \\
\frac{1}{2} & \frac{7}{8} & -\frac{1}{8} \\
-\frac{1}{2} & \frac{1}{8} & \frac{9}{8}
\end{array}\right)
$$

where $\{X, Y, Z\}$ is the coordinate system associated to the basis $\{-I J, J, I\}$. The maps $\rho_{x}^{\prime}(a)$ and $\rho_{x}^{\prime}(b)$ are parabolic rotations on $H_{0} \cong E^{1,2}$ around the nullvector axes $A^{-}$and $B^{-}$. See Figure 14 ,

Case 5: Region (2.2). $x \in(-\infty,-1) \cup(1, \infty) \Longleftrightarrow\left\{1-x^{2}<0,\left(1-x^{2}\right)^{2}<y^{2}\right\}$ $(y>0)$.

There exists an irreducible c-representation $\rho_{x}: G(3 / 1) \longrightarrow S L(2, \mathbb{R})=$ $U_{1} \subset\left(\frac{-1,1}{\mathbb{R}}\right)$ realizing $(x, y)$, unique up to conjugation in $S L(2, \mathbb{R})$, such that

$$
\begin{aligned}
& \rho_{x}(a)=A=x+\sqrt{x^{2}-1} J, \quad \sqrt{x^{2}-1}>0 \\
& \rho_{x}(b)=B=x-\frac{1}{2} \sqrt{\frac{4 x^{2}-3}{x^{2}-1}} I-\frac{2 x^{2}-1}{2 \sqrt{x^{2}-1}} J
\end{aligned}
$$

The composition of $\rho_{x}$ with $c: S L(2, \mathbb{R}) \rightarrow S O^{+}(1,2) \cong I s O^{+}\left(\mathbb{H}^{2}\right)$, define the representations $\rho_{x}^{\prime}=c \circ \rho_{x}: G(3 / 1) \longrightarrow S O^{+}(1,2)$ such that the matrices of $\rho_{x}^{\prime}(a)$ and $\rho_{x}^{\prime}(b)$ are respectively:

$$
\begin{gathered}
m_{x}(a)=\left(\begin{array}{cccc}
2 x^{2}-1 & 0 & 2 x \sqrt{x^{2}-1} & 0 \\
0 & 1 & 0 & \frac{\left(3-4 x^{2}\right) \sqrt{x^{2}-1}}{4 x} \\
2 x \sqrt{x^{2}-1} & 0 & 2 x^{2}-1 & 0
\end{array}\right) \\
m_{x}(b)=\left(\begin{array}{ccc}
2 x^{2}-1 & x \sqrt{\frac{-3+4 x^{2}}{x^{2}-1}} & -\frac{x\left(2 x^{2}-1\right)}{\sqrt{x^{2}-1}} \\
-x \sqrt{\frac{-3+4 x^{2}}{x^{2}-1}} & \frac{1-2 x^{2}}{2 x^{2}-2} & \frac{\sqrt{-3+4 x^{2}\left(2 x^{2}-1\right)}}{2\left(x^{2}-1\right)} \\
-\frac{x\left(2 x^{2}-1\right)}{\sqrt{x^{2}-1}} & \frac{\sqrt{-3+4 x^{2}\left(2 x^{2}-1\right)}}{2\left(x^{2}-1\right)} & \frac{1+2 x^{2}-4 x^{4}}{2-2 x^{2}}
\end{array}\right)
\end{gathered}
$$

The maps $\rho_{x}^{\prime}(a)$ and $\rho_{x}^{\prime}(b)$ are hyperbolic rotations on $H_{0} \cong E^{1,2}$ moving $\delta$ along the polars of the space-like vectors $A^{-}$and $B^{-}$where $x=A^{+}=$ $B^{+}=\cosh \frac{\delta}{2}$. See Figure 9. The distance $d$ (measured in the hyperbolic plane) between the polars of the axes of $\rho_{x}^{\prime}(a)$ and $\rho_{x}^{\prime}(b)$ is given by

$$
\cosh d=\frac{y}{x^{2}-1}=\frac{x^{2}-\frac{1}{2}}{x^{2}-1}
$$

4.2.2. The Figure Eight knot. The algebraic variety $V\left(\mathcal{I}_{G\left(4_{1}\right)}\right)$ for the Figure Eight knot group

$$
G\left(4_{1}\right)=|a, b ; a w=w b|, \quad w=b a^{-1} b^{-1} a,
$$

is defined by the ideal $\mathcal{I}_{G\left(4_{1}\right)}=\left(1-6 x^{2}+4 x^{4}-2 y-2 y^{2}\right)$. See Example 5 , The real part of the algebraic variety $V\left(\mathcal{I}_{G\left(4_{1}\right)}\right)$ is the curve $\mathcal{C}$ depicted in Figure 18, together with the two parabolas $y= \pm\left(x^{2}-1\right)$ of the variety of reducible representations $V\left(y^{2}-\left(x^{2}-1\right)^{2}\right)$.

Figure 19 shows $\frac{y}{1-x^{2}}$ as a function of $x$ for the algebraic variety $V\left(\mathcal{I}_{G\left(4_{1}\right)}\right)$. As before, it is enough to consider this Figure over the pattern of Figure [15] to 


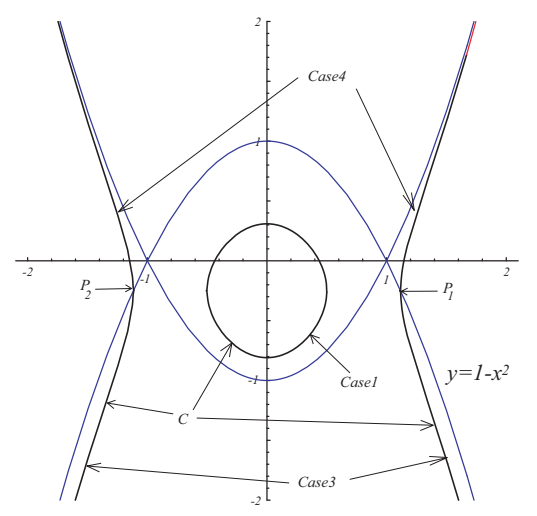

FigURE 18. The real part of $V\left(\mathcal{I}_{G\left(4_{1}\right)}\right)$.

classify the different classes of representation of the group $G\left(4_{1}\right)$ in $S^{3}$ or $S L(2, \mathbb{R})$ according to Theorem 4

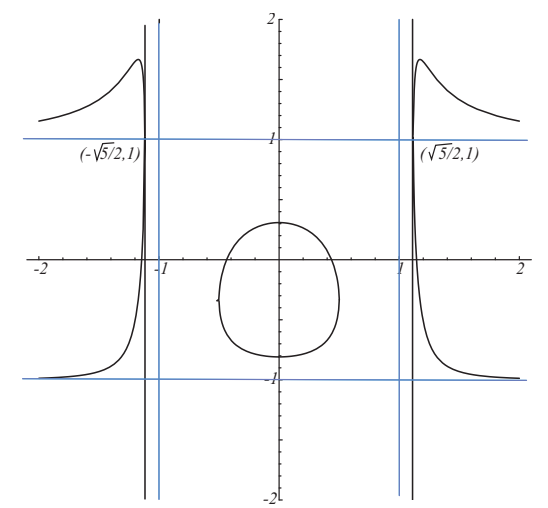

Figure 19. The function $\frac{y}{1-x^{2}}$ for the Figure Eight knot.

There are four cases:

Case 1: Region (1). $x \in\left[-\frac{1}{2}, \frac{1}{2}\right] \Longleftrightarrow\left\{1-x^{2}>0,\left(1-x^{2}\right)^{2}>y^{2}\right\}$. There are two different $y$ values for each value of $x$, except for $x= \pm 1 / 2$ where they coincide.

$$
\begin{aligned}
& y_{1}=\frac{1}{4}\left(-1+\sqrt{5-24 x^{2}+16 x^{4}}\right) \\
& y_{2}=\frac{1}{4}\left(-1-\sqrt{5-24 x^{2}+16 x^{4}}\right)
\end{aligned}
$$

There exists an irreducible c-representation $\rho_{x i}: G\left(4_{1}\right) \longrightarrow S^{3}$ realizing $\left(x, y_{i}\right), i=1,2$, unique up to conjugation in $S^{3}$, such that

$$
\begin{gathered}
\rho_{x 1}(a)=A=x+\frac{-1+\sqrt{5-24 x^{2}+16 x^{4}}}{4 \sqrt{1-x^{2}}} i+\frac{1}{2} \sqrt{\frac{5-4 x^{2}+\sqrt{5-24 x^{2}+16 x^{4}}}{2-x^{2}}} j \\
\rho_{x 1}(b)=B=x+\sqrt{1-x^{2}} i, \quad \sqrt{1-x^{2}}>0 \\
\rho_{x 2}(a)=A=x+\frac{-1-\sqrt{5-24 x^{2}+16 x^{4}}}{4 \sqrt{1-x^{2}}} i+\frac{1}{2} \sqrt{\frac{5-4 x^{2}-\sqrt{5-24 x^{2}+16 x^{4}}}{2-x^{2}}} j \\
\rho_{x 2}(b)=B=x+\sqrt{1-x^{2}} i, \quad \sqrt{1-x^{2}}>0
\end{gathered}
$$

The composition of $\rho_{x i}$ with $c: S^{3} \rightarrow S O(3)$, where $c(X), X \in S^{3}$, acts on $P \in H_{0} \cong E^{3}$ by conjugation, defines the representation $\rho_{x i}^{\prime}=c \circ \rho_{x i}$ : 
$G(3 / 1) \longrightarrow S O(3)$. The maps $\rho_{x i}^{\prime}(a)$ and $\rho_{x i}^{\prime}(b)$ are right rotations of angle $\alpha$ around the axes $A^{-}$and $B^{-}$where $x=A^{+}=B^{+}=\cos \frac{\alpha}{2}$.

The angle $\omega_{i}$ between the axes of $\rho_{x i}^{\prime}(a)$ and $\rho_{x i}^{\prime}(b)$ is given by $\cos \omega_{i}=\frac{y_{i}}{u}$

$$
\begin{aligned}
& \cos \omega_{1}=\frac{y_{1}}{u}=\frac{\frac{1}{4}\left(-1+\sqrt{5-24 x^{2}+16 x^{4}}\right)}{1-x^{2}} \\
& \cos \omega_{2}=\frac{y_{2}}{u}=\frac{\frac{1}{4}\left(-1-\sqrt{5-24 x^{2}+16 x^{4}}\right)}{1-x^{2}}
\end{aligned}
$$

Case 2: Segment $(2.4)(x, y)=\left( \pm \frac{\sqrt{5}}{2},-\frac{1}{4}\right) \Longleftrightarrow\left\{1-x^{2}<0,\left(1-x^{2}\right)^{2}=y^{2}\right\}$. There exists an almost-irreducible c-representation $\rho_{x}: G\left(4_{1}\right) \longrightarrow U_{1} \subset$ $\left(\frac{-1,1}{\mathbb{R}}\right)$ realizing $(x, y)$, unique up to conjugation in $U_{1}$, such that:

$$
\begin{aligned}
& \rho_{ \pm \sqrt{5} / 2}(a)=A=\frac{ \pm \sqrt{5}}{2}+\frac{1}{2} J \\
& \rho_{ \pm \sqrt{5} / 2}(b)=B=\frac{ \pm \sqrt{5}}{2}+I+\frac{1}{2} J+I J
\end{aligned}
$$

Under the isomorphism $U_{1} \approx S L(2, \mathbb{R})$ we have

$$
\begin{aligned}
& \rho_{ \pm \sqrt{5} / 2}: G\left(4_{1}\right) \quad \longrightarrow \quad S L(2, \mathbb{R}) \\
& a \rightarrow A=\left(\begin{array}{cc}
1+\frac{ \pm \sqrt{5}}{2} & \frac{3}{2} \\
-\frac{1}{2} & -1+\frac{ \pm \sqrt{5}}{2}
\end{array}\right) \\
& b \quad \rightarrow \quad B=\left(\begin{array}{cc}
\frac{ \pm \sqrt{5}}{2} & \frac{1}{2} \\
\frac{1}{2} & \frac{ \pm \sqrt{5}}{2}
\end{array}\right)
\end{aligned}
$$

The composition of $\rho_{ \pm \sqrt{5} / 2}$ with $c: S L(2, \mathbb{R}) \rightarrow S O^{+}(1,2) \cong \operatorname{Iso}^{+}\left(\mathbb{H}^{2}\right)$, where $c(X), X \in S L(2, \mathbb{R})$, acts on $P \in H_{0} \cong E^{1,2}$ by conjugation, defines the representation $\rho_{ \pm \sqrt{5} / 2}^{\prime}=c \circ \rho_{ \pm \sqrt{5} / 2}: G(3 / 1) \longrightarrow S O^{+}(1,2)$ in affine linear notation, where $\{X, Y, Z\}$ is the coordinate system associated to the basis $\{-I J, J, I\}$

$$
\begin{aligned}
\rho_{ \pm \sqrt{5} / 2}^{\prime}(a)=m_{ \pm \sqrt{5} / 2}(a)\left(\begin{array}{c}
X \\
Y \\
Z
\end{array}\right)= & \left(\begin{array}{c}
X^{\prime} \\
Y^{\prime} \\
Z^{\prime}
\end{array}\right) \\
\rho_{ \pm \sqrt{5} / 2}^{\prime}(b)=m_{ \pm \sqrt{5} / 2}(b)\left(\begin{array}{c}
X \\
Y \\
Z
\end{array}\right)= & \left(\begin{array}{c}
X^{\prime} \\
Y^{\prime} \\
Z^{\prime}
\end{array}\right)
\end{aligned}
$$

where the matrices of $\rho_{ \pm \sqrt{5} / 2}^{\prime}(a)$ and $\rho_{ \pm \sqrt{5} / 2}^{\prime}(b)$ are respectively:

$$
m_{ \pm \sqrt{5} / 2}(a)=\left(\begin{array}{ccc}
\frac{3}{2} & 0 & \frac{\sqrt{5}}{2} \\
0 & 1 & 0 \\
\frac{\sqrt{5}}{2} & 0 & \frac{3}{2}
\end{array}\right)
$$

and

$$
m_{ \pm \sqrt{5} / 2}(b)=\left(\begin{array}{ccc}
-\frac{1}{2} & 1-\sqrt{5} & \frac{1}{2}(\sqrt{5}-4) \\
1+\sqrt{5} & 1 & 1+\sqrt{5} \\
\frac{1}{2}(\sqrt{5}+4) & -1+\sqrt{5} & \frac{7}{2}
\end{array}\right)
$$

The maps $\rho_{x}^{\prime}(a)$ and $\rho_{x}^{\prime}(b)$ are hyperbolic rotations on $H_{0} \cong E^{1,2}$ around the space-like axes $A^{-}$and $B^{-}$, where $x=A^{+}=B^{+}=\cosh \frac{d}{2}$. See Figure 13 .

Case 3: Region $(2.2)|x|>1,\left(1-x^{2}\right)^{2}<y^{2}$. This case consists of the real points $(x, y) \in V\left(\mathcal{I}_{G}\right)$ where $|x|>1$ and $y=\frac{1}{4}\left(-1-\sqrt{5-24 x^{2}+16 x^{4}}\right) \leq$ $-\frac{1}{4}$. 
There exists an irreducible c-representation $\rho_{x}: G(3 / 1) \longrightarrow S L(2, \mathbb{R})=$ $U_{1} \subset\left(\frac{-1,1}{\mathbb{R}}\right)$ realizing $(x, y)$, unique up to conjugation in $S L(2, \mathbb{R})$, such that

$$
\begin{aligned}
& \rho_{x}(a)=A=x+\sqrt{x^{2}-1} J, \quad \sqrt{x^{2}-1}>0 \\
& \rho_{x}(b)=B=x+\frac{1}{2} \sqrt{\frac{5-4 x^{2}-\sqrt{5-24 x^{2}+16 x^{4}}}{2 x^{2}-2}} I+\frac{1+\sqrt{5-24 x^{2}+16 x^{4}}}{4 \sqrt{x^{2}-1}} J
\end{aligned}
$$

The composition of $\rho_{x}$ with $c: S L(2, \mathbb{R}) \rightarrow S O^{+}(1,2) \cong I s o^{+}\left(\mathbb{H}^{2}\right)$, where $c(X), X \in S L(2, \mathbb{R})$, acts on $P \in H_{0} \cong E^{1,2}$ by conjugation, defines the representation $\rho_{x}^{\prime}=c \circ \rho_{x}: G(3 / 1) \longrightarrow S O^{+}(1,2)$.

The maps $\rho_{x}^{\prime}(a)$ and $\rho_{x}^{\prime}(b)$ are hyperbolic rotations on $H_{0} \cong E^{1,2}$ around the space-like axes $A^{-}$and $B^{-}$, where $x=A^{+}=B^{+}=\cosh \frac{d}{2}$. See Figure 9.

The distance $\delta$ between the polars of the axes of $\rho_{x}^{\prime}(a)$ and $\rho_{x}^{\prime}(b)$ (measured in the hyperbolic plane) is given by

$$
\cosh \delta=\frac{-y}{u}=\frac{-\frac{1}{4}\left(-1-\sqrt{5-24 x^{2}+16 x^{4}}\right)}{x^{2}-1}>1
$$

Case 4: Region (2.3) $|x|>1,\left(1-x^{2}\right)^{2}>y^{2}$. This case consists of the real points $(x, y) \in V\left(\mathcal{I}_{G}\right)$ where $|x|>1$ and $y=\frac{1}{4}\left(-1+\sqrt{5-24 x^{2}+16 x^{4}}\right) \geq$ $-\frac{1}{4}$.

There exists an irreducible c-representation $\rho_{x}: G(3 / 1) \longrightarrow S L(2, \mathbb{R})=$ $U_{1} \subset\left(\frac{-1,1}{\mathbb{R}}\right)$ realizing $(x, y)$, unique up to conjugation in $S L(2, \mathbb{R})$, such that

$$
\begin{aligned}
& \rho_{x}(a)=A=x+\sqrt{x^{2}-1} J \\
& \rho_{x}(b)=B=x+\frac{1-\sqrt{5-24 x^{2}+16 x^{4}}}{4 \sqrt{x^{2}-1}} J+\frac{1}{2} \sqrt{\frac{5-4 x^{2}+\sqrt{5-24 x^{2}+16 x^{4}}}{2 x^{2}-2}} I J
\end{aligned}
$$

The composition of $\rho_{x}$ with $c: S L(2, \mathbb{R}) \rightarrow S O^{0}(1,2) \cong I s O^{+}\left(\mathbb{H}^{2}\right)$, where $c(X), X \in S L(2, \mathbb{R})$, acts on $P \in H_{0} \cong E^{1,2}$ by conjugation, defines the representation $\rho_{x}^{\prime}=c \circ \rho_{x}: G(3 / 1) \longrightarrow S O^{0}(1,2)$.

The maps $\rho_{x}^{\prime}(a)$ and $\rho_{x}^{\prime}(b)$ are hyperbolic rotations on $H_{0} \cong E^{1,2}$ around the space-like vectors $A^{-}$and $B^{-}$, where $x=A^{+}=B^{+}=\cosh \frac{d}{2}$. See Figure 11 .

The angle $\theta$ (measured in the hyperbolic plane) between the polars of the axes of $\rho_{x}^{\prime}(a)$ and $\rho_{x}^{\prime}(b)$ is given by

$$
\cos \theta=\frac{-y}{x^{2}-1}=\frac{1-\sqrt{5-24 x^{2}+16 x^{4}}}{4\left(x^{2}-1\right)}
$$

We have omitted the expression of $\rho_{x}^{\prime}(a)$ and $\rho_{x}^{\prime}(b)$ in affine linear notation, where $\{X, Y, Z\}$ is the coordinate system associated to the basis $\{-i j, j, i\}$ for the above cases 1, 3 and 4 , but they can be easily obtained using the equation (2.3) of $\S 2.3 .1$.

\section{Groups of isometries in a QUATERnion ALGEBra H}

Let $H=\left(\frac{\mu, \nu}{k}\right)$ be a quaternion algebra. The pure quaternions form a 3 dimensional vector space $H_{0}$.

The following map is a left action of the group $U$ on the 3-dimensional vector space $H_{0}$.

$$
\begin{aligned}
& \Phi: U \times H_{0} \quad \longrightarrow \quad H_{0} \\
& \left(A, B^{-}\right) \quad \rightarrow \quad A \circ B^{-}:=A B^{-} \bar{A}
\end{aligned}
$$


The restriction of $\Phi$ to the subgroup $U_{1}$ (the unit quaternions, norm 1 ) is also a left action on the 3 -dimensional vector space $H_{0}$.

$$
\begin{aligned}
\Phi_{0}: & U_{1} \times H_{0} \rightarrow H_{0} \\
& \left(A, B^{-}\right) \rightarrow A \circ B^{-}:=A B^{-} \bar{A}
\end{aligned}
$$

The equiform group or group of similarities $\mathcal{E} q(H)$ of a quaternion algebra $H$, is the semidirect product $H_{0} \rtimes U$. This is the group whose underlying space is $H_{0} \times U$ and the product is

$$
\begin{aligned}
& \mathcal{E} q(H) \times \mathcal{E} q(H) \longrightarrow \mathcal{E}_{q}(H) \\
& ((v, A),(w, B)) \rightarrow(v+A \circ w, A B)
\end{aligned}
$$

The group of affine isometries $A(H)$ of a quaternion algebra is the subgroup of $\mathcal{E} q(H)$ which is the semidirect product $H_{0} \rtimes U_{1}$. This is the group whose underlying space is $H_{0} \times U_{1}$ and the product is

$$
\begin{aligned}
& A(H) \times A(H) \quad \longrightarrow \quad A(H) \\
& ((v, A),(w, B)) \quad \rightarrow \quad(v+A \circ w, A B)
\end{aligned}
$$

The group $\mathcal{E} q(H)$ defines a left action on the 3 -dimensional vector space $H_{0}$,

$$
\begin{aligned}
& \Psi: \quad \mathcal{E} q\left(H_{0}\right) \times H_{0} \quad \longrightarrow \quad H_{0} \\
& ((v, A), u) \quad \rightarrow \quad(v, A) u:=v+A \circ u
\end{aligned}
$$

because

$$
\begin{aligned}
& \Psi((0,1), u)=(0,1) u=0+1 u \overline{1}=u \\
& \Psi\left(\left(v_{2}, A_{2}\right), \Psi\left(\left(v_{1}, A_{1}\right), u\right)\right)=\left(v_{2}, A_{2}\right)\left(v_{1}+A_{1} \circ u\right)=v_{2}+A_{2} \circ\left(v_{1}+A_{1} \circ u\right) \\
&=v_{2}+A_{2} \circ v_{1}+A_{2} A_{1} \circ u=\Psi\left(\left(v_{2}+A_{2} \circ v_{1}, A_{2} A_{1}\right), u\right) \\
&=\Psi\left(\left(v_{2}, A_{2}\right)\left(v_{1}, A_{1}\right), u\right)
\end{aligned}
$$

The restriction of $\Psi$ to the subgroup $A(H)$ is also a left action on the 3dimensional vector space $H_{0}$.

For an element $(v, A) \in \mathcal{E} q(H), A$ is the linear part of $(v, A), N(A)$ is the homothetic factor, and $v$ is the translational part. Note that if $(v, A) \in A(H)$, then the homothetic factor is 1 . The action of each element $(v, A) \in A(H)$ in $H_{0}$ is called an affine isometry.

Example 6. 1.- $H=\left(\frac{-1,-1}{\mathbb{R}}\right)=\mathbb{H}$. Then $\mathcal{E} q(H)$ is the group of affine isometries and similarities of the Euclidean 3-dimensional space $E^{3}$. The linear part of $\mathcal{E} q(H)$ is the multiplicative group $(\mathbb{H} \backslash\{0\})$ of the algebra $\mathbb{H}$. The subgroup $A(H)$ is the group of orientation preserving affine isometries of $E^{3}$. It is called the Euclidean group and we denote it by $\mathcal{E}\left(\mathbb{R}^{3}\right)$. The linear part of $A(H)$ is $S O(3, \mathbb{R})$.

2.- $H=\left(\frac{-1,1}{\mathbb{R}}\right)=M(2, \mathbb{R})$. Then $\mathcal{E} q(H)$ is the group of affine isometries and similarities of the Minkowski 3-dimensional space $E^{1,2}$. The linear part of $\mathcal{E} q(H)$ is isomorphic to $G l(2, \mathbb{R})$. The subgroup $A(H)$ is the group of orientation preserving affine isometries of $E^{1,2}$. It is called the Lorentz group and we denote it by $\mathcal{L}\left(\mathbb{R}^{3}\right)$. The linear part is $S O^{0}(1,2)$.

3.- $H=\left(\frac{-1,1}{\mathbb{C}}\right)=M(2, \mathbb{C})$. Then $\mathcal{E} q(H)$ is the group of affine isometries and similarities of the complex 3-dimensional space $\mathbb{C}^{3}$. The linear part of $\mathcal{E} q(H)$ is isomorphic to $G l(2, \mathbb{C})$. Here $A(H)$ is the subgroup of orientation preserving affine isometries of $\mathbb{C}^{3}$. The linear part of $A(H)$ is $S O(3, \mathbb{C})$. 
5.0.3. Axis and shift of an element $(v, A) \in A(H)$. Consider $(v, A) \in A(H)$. The vector $v \in H_{0}$ can be decomposed in a unique way as the orthogonal sum of two vectors, one of them in the $A^{-}$direction:

$$
v=s A^{-}+v^{\perp}, \quad\left\langle v^{\perp}, A^{-}\right\rangle=0
$$

Then

$$
(v, A)=\left(v^{\perp}, 1\right)\left(s A^{-}, A\right)
$$

The element $\left(v^{\perp}, 1\right)$ is a translation in $H_{0}$. The restriction of the action of $\left(s A^{-}, A\right)$ on the line generated by $A^{-}$is a translation with vector $s A^{-}$:

$$
\left(s A^{-}, A\right)\left(\lambda A^{-}\right)=s A^{-}+A\left(\lambda A^{-}\right) \bar{A}=s A^{-}+\lambda A^{-}=(s+\lambda) A^{-}
$$

We define $s A^{-}$as the vector shift of the element $(v, A)$. The length $\sigma$ of the vector shift will be called the shift of the element $(A, v)$.

The action of $(v, A)$ leaves (globally) invariant an affine line parallel to $A^{-}$and its action on this line is a translation with vector the shift $s A^{-}$. This invariant affine line will be call the axis of $(v, A)$. Then the action of $(v, A)$ on the axis of $(v, A)$ is a translation by $\sigma$. It is easy to see that the axis of $(v, A)$ is $\left\{u+\mu A^{-}: \mu \in k\right\}$ where $u$ is defined by $u-A \circ u=v^{\perp}$. In fact,

$$
\begin{gathered}
(v, A)\left(u+\mu A^{-}\right)=u+(\mu+s) A^{-} \\
\Longrightarrow \quad v+A \circ\left(u+\mu A^{-}\right)=u+(\mu+s) A^{-} \\
\Longrightarrow \quad s A^{-}+v^{\perp} \quad+A \circ u+\mu A^{-}=u+(\mu+s) A^{-} \\
\Longrightarrow \quad u-A \circ u=v^{\perp}+(s+\mu-(\mu+s)) A^{-}=v^{\perp}
\end{gathered}
$$

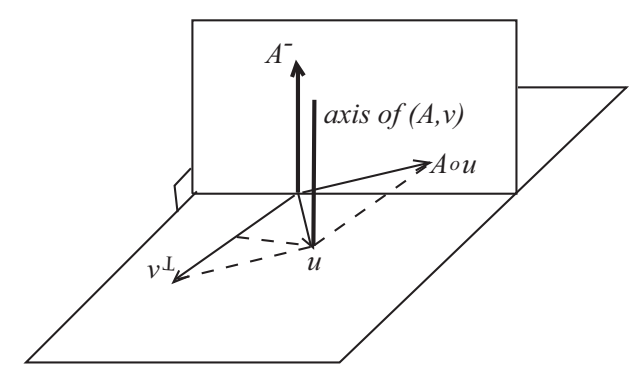

Figure 20. The axis of $(A, v)$.

Remark 9. Observed that $\left(v_{1}, A\right)$ and $\left(v_{2}, A\right)$ have the same axis if and only if $v_{1}^{\perp}=v_{2}^{\perp}$. Therefore, given an element $(v, A)$, the element $\left(v^{\perp}, A\right)$, where $v=$ $s A^{-}+v^{\perp},\left\langle v^{\perp}, A^{-}\right\rangle=0$, has the same axis that $(v, A)$ but shift zero.

5.1. Two conjugate elements of $A(H)$. If $(v, A),(w, B) \in A(H)$ are conjugate elements in $A(H)$ then $A$ and $B$ are conjugate in $U_{1}$ and the vector shifts of $(v, A)$ and $(w, B)$ are respectively $s A^{-}$and $s B^{-}$. In fact, a pair $(v, A),(w, B) \in A(H)$ of conjugate elements in $A(H)$, is conjugate to the pair $\left(s A^{-}, A\right),\left(s B^{-}+w^{\perp}, B\right)$, where $\left\langle w^{\perp}, B^{-}\right\rangle=0$. This conjugation is made by a translation (change of the origin point in $H_{0}$ ).

Lemma 3. If the elements $\left(s A^{-}, A\right),\left(s B^{-}+w^{\perp}, B\right)$, where $\left\langle w^{\perp}, B^{-}\right\rangle=0$, are conjugate elements in $A(H)$ or $\mathcal{E} q(H)$ by an element $(u, C)$ then, for any $\lambda \in k$, $\left(\lambda s A^{-}, A\right),\left(\lambda s B^{-}+w^{\perp}, B\right)$ are also conjugate by the same element.

Proof. Let $(u, C) \in A(H)$ or $\mathcal{E} q(H)$ be the element such that

$$
(u, C)\left(s A^{-}, A\right)(u, C)^{-1}=\left(s B^{-}+w^{\perp}, B\right)
$$


Then

$$
\begin{aligned}
(u, C)\left(s A^{-}, A\right)(u, C)^{-1}= & (u, C)\left(s A^{-}, A\right)\left(-C^{-1} \circ u, C^{-1}\right) \\
= & (u, C)\left(s A^{-}-\left(A C^{-1}\right) \circ u, A C^{-1}\right) \\
= & \left(u+C \circ s A^{-}-\left(C A C^{-1}\right) \circ u, C A C^{-1}\right) \\
= & \left(u+s C A^{-} C^{-1}-\left(C A C^{-1}\right) \circ u, C A C^{-1}\right) \\
= & \left(s B^{-}+w^{\perp}, B\right)
\end{aligned}
$$

implies that

$$
\begin{aligned}
C A C^{-1} & =B \\
u-C A C^{-1} \circ u & =w^{\perp}
\end{aligned}
$$

Then

$$
(u, C)\left(\lambda s A^{-}, A\right)(u, C)^{-1}=\left(\lambda s B^{-}+w^{\perp}, B\right)
$$

Proposition 11. If $(A, B)$ is a irreducible pair, that is $\left\{A^{-}, B^{-},\left(A^{-} B^{-}\right)^{-}\right\}$is a basis of $H_{0}$, then the axes of $\left(s A^{-}, A\right)$ and $\left(s B^{-}+\left(A^{-} B^{-}\right)^{-}, B\right)$ do not intersect.

Proof. By Remark 9, the axes of $\left(s A^{-}, A\right)$ and $(0, A)$ are both the vector line generated by $A^{-}$. By the same reason the axes of $\left(s B^{-}+\left(A^{-} B^{-}\right)^{-}, B\right)$ and $\left(\left(A^{-} B^{-}\right)^{-}, B\right)$ also coincide. By (5.1) , the axis of $\left(\left(A^{-} B^{-}\right)^{-}, B\right)$ is $\left\{u+\mu B^{-}, \mu \in k\right\}$, such that $u-B \circ u=\left(A^{-} B^{-}\right)^{-}$. Let us prove by contradiction that $u$ is not contained in the plane generated by $\left\{A^{-}, B^{-}\right\}$, where the axis of $(0, A)$ lives. Assume that $u$ belongs to the plane generated by $\left\{A^{-}, B^{-}\right\}$, then by Corollary 1 $u \perp\left(A^{-} B^{-}\right)^{-},\left\langle u,\left(A^{-} B^{-}\right)^{-}\right\rangle=0$. Thus, since $B$ is an isometry,

$$
\begin{aligned}
\langle u, u\rangle & =\langle B \circ u, B \circ u\rangle=\left\langle u-\left(A^{-} B^{-}\right)^{-}, u-\left(A^{-} B^{-}\right)^{-}\right\rangle \\
& =\langle u, u\rangle+\left\langle\left(A^{-} B^{-}\right)^{-},\left(A^{-} B^{-}\right)^{-}\right\rangle \\
& \Longrightarrow\left\langle\left(A^{-} B^{-}\right)^{-},\left(A^{-} B^{-}\right)^{-}\right\rangle=N\left(\left(A^{-} B^{-}\right)^{-}\right)=0
\end{aligned}
$$

Then $\left(A^{-} B^{-}\right)^{-} \in\left(\left(A^{-} B^{-}\right)^{-}\right)^{\perp}$. But the plane $\left(\left(A^{-} B^{-}\right)^{-}\right)^{\perp}$ is the plane generated by $\left\{A^{-}, B^{-}\right\}$, which is impossible because $\left\{A^{-}, B^{-},\left(A^{-} B^{-}\right)^{-}\right\}$is a basis. Therefore the axis $\left\{\lambda A^{-}: \lambda \in k\right\}$ is contained in the plane $\Pi$ generated by $\left\{A^{-}, B^{-}\right\}$, and the axis $\left\{u+\mu B^{-}: \mu \in k\right\}$ is contained in the plane $u+\Pi$ parallel to $\Pi$ but different from $\Pi$. Then both axes do not intersect.

Next, we will prove that if $\left\{A^{-}, B^{-},\left(A^{-} B^{-}\right)^{-}\right\}$is a basis in $H_{0}$ and $(v, A),(w, B) \in$ $A(H)$ is a pair of conjugate elements whose axes do not intersect, it is possible to conjugate them in $\mathcal{E} q(H)$ to the standard elements of the above Proposition 11.

Theorem 5. Let $(v, A),(w, B) \in A(H)$ be a pair of conjugate elements in $A(H)$ whose axes do not intersect. Assume $(A, B)$ is a irreducible pair. Then the pair $((v, A),(w, B)) \in A(H)$ is conjugate in $\mathcal{E} q(H)$ to a pair of the form $\left(\left(s A^{-}, A\right)\right.$, $\left.\left(s B^{-}+\left(A^{-} B^{-}\right)^{-}, B\right)\right)$ or $\left(\left(s A^{-}, A\right),\left(s B^{-}-\left(A^{-} B^{-}\right)^{-}, B\right)\right)$.

Proof. Up to conjugation by a translation (change of the origin point in $H_{0}$ ) we can assume that $(v, A)=\left(t A^{-}, A\right)$, and $(w, B)=\left(t B^{-}+w^{\perp}, B\right)$, where $w^{\perp} \perp B^{-}$.

The idea is to apply a conjugation by an element of $\mathcal{E} q(H)$ to a pair of the form $\left(\left(s A^{-}, A\right),\left(s B^{-} \pm\left(A^{-} B^{-}\right)^{-}, B\right)\right)$ to obtain the pair $\left(\left(t A^{-}, A\right),\left(t B^{-}+w^{\perp}, B\right)\right)$. Note that conjugating by the translations $\left\{\left(\lambda A^{-}, 1\right), \lambda \in k\right\}$ does not change the axis of $\left(s A^{-}, A\right)$. However the axis of $\left.\left(s B^{-} \pm\left(A^{-} B^{-}\right)^{-}, B\right)\right)$ generates a family $\mathcal{F}$ of non intersecting lines whose union is two planes $\mathcal{P}$ parallel to the plane $\Pi=\left\{A^{-}, B^{-}\right\}$ and placed on both sides of it. Conjugating by the set of homothetic transformations $\left\{(0, r I), r \in k^{*}\right\}$ the axis of $\left(s A^{-}, A\right)$ does not change, but the family $\mathcal{F}$ contained 
in $\mathcal{P}$ generates a family of non intersecting lines whose union is the complement of the plane $\Pi=\left\{A^{-}, B^{-}\right\}$. See Figure 21]

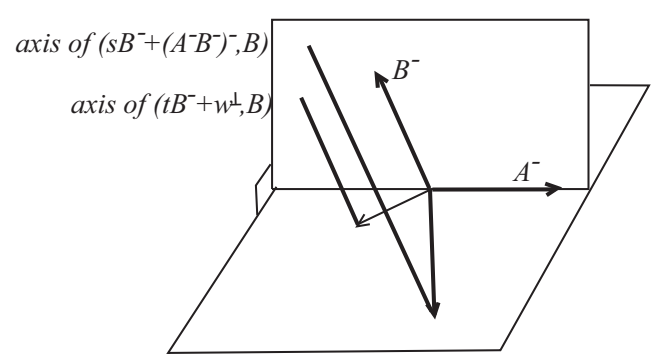

Figure 21. Axes of $(w, B)$ and $\left.\left(s B^{-}+\left(A^{-} B^{-}\right)^{-}, B\right)\right)$.

Actually, we can find an element $\left(\lambda A^{-}, r I\right) \in \mathcal{E} q(H)$ such that

$$
\left(\lambda A^{-}, r I\right)\left(s A^{-}, A\right)\left(\lambda A^{-}, r I\right)^{-1}=\left(r^{2} s A^{-}, A\right)
$$

$$
\left(\lambda A^{-}, r I\right)\left(s B^{-} \pm\left(A^{-} B^{-}\right)^{-}, B\right)\left(\lambda A^{-}, r I\right)^{-1}=\left(r^{2} s B^{-}+w^{\perp}, B\right)
$$

The first equation (5.2) is always true. From the second one (5.3) we can obtain the values of $r$ and $\lambda$ :

$$
\begin{aligned}
& \left(\lambda A^{-}, r I\right)\left(s B^{-} \pm\left(A^{-} B^{-}\right)^{-}, B\right)\left(\lambda A^{-}, r I\right)^{-1} \\
= & \left(\lambda A^{-}, r I\right)\left(s B^{-} \pm\left(A^{-} B^{-}\right)^{-}, B\right)\left(-\left(\frac{1}{r} I\right) \circ\left(\lambda A^{-}\right), \frac{1}{r} I\right) \\
= & \left(\lambda A^{-}, r I\right)\left(s B^{-} \pm\left(A^{-} B^{-}\right)^{-}-B \circ\left(\frac{1}{r^{2}} \lambda A^{-}\right), \frac{1}{r} B\right) \\
= & \left(\lambda A^{-}+r^{2}\left(s B^{-} \pm\left(A^{-} B^{-}\right)^{-}-\frac{\lambda}{r^{2}} B A^{-} \bar{B}, B\right)=(v, B)\right.
\end{aligned}
$$

where

$$
v=\lambda A^{-}+r^{2}\left(s B^{-} \pm\left(A^{-} B^{-}\right)^{-}-\frac{\lambda}{r^{2}} B A^{-} \bar{B}\right.
$$

Let us compute $B A^{-} \bar{B}$ in the basis $\left\{A^{-}, B^{-},\left(A^{-} B^{-}\right)^{-}\right\}$

$B A^{-} \bar{B}=\left(B^{+}+B^{-}\right) A^{-}\left(B^{+}-B^{-}\right)=\left(B^{+}\right)^{2} A^{-}+B^{+}\left(B^{-} A^{-}-A^{-} B^{-}\right)-B^{-} A^{-} B^{-}$ Using the equation

$$
A^{-} B^{-}+B^{-} A^{-}=2\left(A^{-} B^{-}\right)^{+}
$$

and the notation

$$
\begin{aligned}
& x=B^{+} \\
& u=-B^{-} B^{-}=1-x^{2} \\
& y=-\left(A^{-} B^{-}\right)^{+}
\end{aligned}
$$

We have

$B A^{-} \bar{B}=x^{2} A^{-}-2 x\left(A^{-} B^{-}\right)^{-}+2 y B^{-}-u A^{-}=\left(2 x^{2}-1\right) A^{-}+2 y B^{-}-2 x\left(A^{-} B^{-}\right)^{-}$

Therefore

$$
v=\lambda\left(2-2 x^{2}\right) A^{-}+\left(r^{2} s-2 \lambda y\right) B^{-}+\left( \pm r^{2}-2 \lambda x\right)\left(A^{-} B^{-}\right)^{-}
$$

and if

$$
(v, B)=\left(r^{2} s B^{-}+w^{\perp}, B\right)
$$

then

$$
\begin{aligned}
v & =r^{2} s B^{-}+w^{\perp} \Longrightarrow \\
w^{\perp} & =\lambda\left(2-2 x^{2}\right) A^{-}-2 \lambda y B^{-}+\left( \pm r^{2}-2 \lambda x\right)\left(A^{-} B^{-}\right)^{-}
\end{aligned}
$$


where $w^{\perp}=a A^{-}+b B^{-}+c\left(A^{-} B^{-}\right)^{-}$is such that $\left\langle w^{\perp}, B^{-}\right\rangle=0$. Thus

$$
\begin{aligned}
0 & =\left\langle a A^{-}+b B^{-}+c\left(A^{-} B^{-}\right)^{-}, B^{-}\right\rangle \\
& =a\left\langle A^{-}, B^{-}\right\rangle+b\left\langle B^{-}, B^{-}\right\rangle+c\left\langle\left(A^{-} B^{-}\right)^{-}, B^{-}\right\rangle=a y+b\left(1-x^{2}\right)
\end{aligned}
$$

We obtain the system

$$
\begin{aligned}
a & =\lambda\left(2-2 x^{2}\right) \\
b & =-2 \lambda y \\
c & = \pm r^{2}-2 \lambda x
\end{aligned}
$$

Then

$$
\begin{aligned}
& \lambda=\frac{a}{2-2 x^{2}} \\
& \pm r^{2}=c+\frac{a x}{1-x^{2}}
\end{aligned}
$$

Here, if $k=\mathbb{R}$, two cases are possible:

$$
\begin{aligned}
& c+\frac{a x}{1-x^{2}}>0, \text { take the }+ \text { sign, } r=\sqrt{c+\frac{a x}{1-x^{2}}} \\
& c+\frac{a x}{1-x^{2}}<0, \text { take the }- \text { sign, } r=\sqrt{-\left(c+\frac{a x}{1-x^{2}}\right)}
\end{aligned}
$$

We have proven that, in the first case, the pair $\left(\left(s A^{-}, A\right),\left(s B^{-}+\left(A^{-} B^{-}\right)^{-}, B\right)\right)$ is conjugate in $\mathcal{E} q(H)$ to the pair $\left(\left(r^{2} s A^{-}, A\right),\left(r^{2} s B^{-}+w^{\perp}, B\right)\right)$, and that in the second case, the pair $\left(\left(s A^{-}, A\right),\left(s B^{-}-\left(A^{-} B^{-}\right)^{-}, B\right)\right)$ is conjugate in $\mathcal{E} q(H)$ to the pair $\left(\left(r^{2} s A^{-}, A\right),\left(r^{2} s B^{-}+w^{\perp}, B\right)\right)$. By Lemma 3 we can deduce that the pair $\left((v, A)=\left(t A^{-}, A\right),(w, B)=\left(t B^{-}+w^{\perp}, B\right)\right)$ is conjugate to the pair $\left(\left(t A^{-}, A\right),\left(t B^{-} \pm\left(A^{-} B^{-}\right)^{-}, B\right)\right)$.

Corollary 2. Let $(v, A),(w, B) \in A(H)$ be a pair of conjugate elements in $A(H)$ whose axes do not intersect. Assume $(A, B)$ is a irreducible pair. Then $(v, A),(w, B)$ are determined up to similarity in $H_{0}$ by the parameters $x, y, s$.

The geometrical meaning of the parameters depends on the geometry of $H_{0}$, that is, on the quaternion algebra $H=\left(\frac{\mu, \nu}{k}\right)$.

5.2. c-Representations in $A(H)$. Let $G=|a, b ; w(a, b)|$ be a presentation of a group. For example, $G=|a, b ; w(a, b)|$ can be a presentation of the group of a 2-bridge knot, where $a$ and $b$ are represented by coherently oriented meridians of the knot. We want to study the c-representations of $G$ in the affine group $A(H)$ of a quaternion algebra $H$, that is representations of $G$ in the affine group $A(H)$ of a quaternion algebra $H$ such that the generators $a$ and $b$ go to conjugate elements, up to conjugation in $\mathcal{E} q(H)$. We have already studied the case of the c-representation whose image lies in the subgroup $U_{1}$ of unit quaternions:

$$
\begin{aligned}
\rho: \quad G & \longrightarrow A(H) \\
a & \rightarrow(0, A) \\
b & \rightarrow(0, B)
\end{aligned}
$$

From now on we will assume that at least one of the elements $\rho(a), \rho(b)$ has translational part different from 0 , and that $(A, B)$ is a irreducible pair of conjugate unit quaternions. By Theorem $[5]$ we may assume that

$$
\begin{aligned}
\rho(a) & =\left(s A^{-}, A\right) \\
\rho(b) & =\left(s B^{-}+\left(A^{-} B^{-}\right)^{-}, B\right)
\end{aligned}
$$



have

Because $\rho$ is an homomorphism of the semidirect product $H_{0} \rtimes U_{1}=A(H)$, we

$$
\rho(w(a, b))=\left(\left.\frac{\partial w}{\partial a}\right|_{\phi} \circ v+\left.\frac{\partial w}{\partial b}\right|_{\phi} \circ u, w(A, B)\right)=(0, I)
$$

where $\left.\frac{\partial w}{\partial a}\right|_{\phi}$ is the Fox derivative of the word $w(a, b)$ with respect to $a$, and evaluated by $\phi$ such that $\phi(a)=A, \phi(b)=B$. (See [4.) The equation (5.4) yields two relations between the parameters

$$
\begin{aligned}
& x=A^{+}=B^{+} \\
& y=-\left(A^{-} B^{-}\right)^{+} \\
& s=\text { vector shift parameter }
\end{aligned}
$$

the relations are

$$
\begin{gathered}
w(A, B)=I \\
\left.\frac{\partial w}{\partial a}\right|_{\phi} \circ v+\left.\frac{\partial w}{\partial b}\right|_{\phi} \circ u=0
\end{gathered}
$$

The relation (5.5) yields the ideal $\mathcal{I}_{G}^{c}=\left\{p_{i}(x, y) \mid i \in\{1,2,3,4\}\right\}$, as we proved in Section 4. It defines $V\left(\mathcal{I}_{G}^{c}\right)$ the algebraic variety of c-representations of $G$ in $S L(2, \mathbb{C})$.

The relation (5.6) produces four polynomials in $x, y, s:\left\{q_{j}(x, y, s) \mid j \in\{1,2,3,4\}\right\}$. The ideal

$$
\mathcal{I}_{a G}^{c}=\left\{p_{i}(x, y), q_{j}(x, y, s) \mid i, j \in\{1,2,3,4\}\right\}
$$

defines an algebraic variety, that we call $V_{a}\left(\mathcal{I}_{a G}^{c}\right)$ the variety of affine c-representations of $G$ in $A(H)$ up to conjugation in $\mathcal{E} q\left(H_{0}\right)$.

Let

$$
\begin{aligned}
\rho: \quad G & \longrightarrow A(H) \\
a & \rightarrow \rho(a)=\left(s A^{-}, A\right) \\
b & \rightarrow \rho(b)=\left(\left(s B^{-}+\left(A^{-} B^{-}\right)^{-}, B\right)\right.
\end{aligned}
$$

be a representation of $G$ in the affine group of a quaternion algebra $H$. The composition of $\rho$ with the projection $\pi_{2}$ on the second factor of $A(H)=H_{0} \rtimes U_{1}$ gives the linear part of $\rho$ and it is a representation $\widehat{\rho}$ on the group of unit quaternions.

$$
\begin{aligned}
\widehat{\rho}=\pi_{2} \circ \rho: & G \longrightarrow U_{0} \\
a & \rightarrow A \\
b & \rightarrow B
\end{aligned}
$$

The composition of $\rho$ with the projection $\pi_{1}$ on the first factor of $A(H)=H_{0} \rtimes U_{1}$ gives the translational part of $\rho$ :

$$
v_{\rho}=\pi_{1} \circ \rho: \quad G \quad \longrightarrow \quad H_{0}
$$

which is called a cocycle because it satisfy the cocycle condition

$$
v_{\rho}\left(g_{1} g_{2}\right)=v_{\rho}\left(g_{1}\right)+\widehat{\rho}\left(g_{1}\right) v_{\rho}\left(g_{2}\right)
$$

Therefore $\widehat{\rho}$ corresponds to a point in the character variety of c-representations of $G$ and it is determined by the characters $x$ and $y$. Reciprocally, given a representation

$$
\widehat{\rho}: G \longrightarrow U_{1}
$$

an affine deformation $\rho$ of $\hat{\rho}$ is a homomorphism $\rho: G \longrightarrow A(H)$ such that

(i) $\rho(g)=\left(v_{g}, \widehat{\rho}(g)\right), \forall g \in G$

(ii) $\quad v_{g} \neq 0$ for some $g \in G$

Observe that every representation $\rho$ of $G$ in the affine group of a quaternion algebra $H$ is an affine deformation of $\widehat{\rho}=\pi_{2} \circ \rho$ if and only if the cocycle $v_{\rho}=\pi_{1} \circ \rho: G \longrightarrow$ $H_{0}$ is not constant. 
We are interested in the classes of affine deformations up to conjugation in $\mathcal{E} q(H)$. Each of these classes is determined by the parameter $s$.

Example 7 (The trefoil knot). Consider the group of the trefoil knot $3_{1}$

$$
G\left(3_{1}\right)=|a, b ; a b a=b a b|
$$

Let

$$
\begin{aligned}
& \rho: G\left(3_{1}\right) \quad \longrightarrow \quad A(H) \\
& a \quad \rightarrow \quad \rho(a)=\left(s A^{-}, A\right) \\
& b \quad \rightarrow \quad \rho(b)=\left(s B^{-}+\left(A^{-} B^{-}\right)^{-}, B\right)
\end{aligned}
$$

be a representation of $G$ in the affine group of a quaternion algebra $H$. Assume that $\left\{A^{-}, B^{-},\left(A^{-} B^{-}\right)^{-}\right\}$is a basis of $H_{0}$.

Then the parameters $x, y, s$ satisfy the equations:

$$
\begin{aligned}
2 y-\left(2 x^{2}-1\right) & =0 \\
4 x^{2}+4 s x-3 & =0
\end{aligned}
$$

Therefore the variety of affine representation of $G\left(3_{1}\right)$ is

$$
V_{a}\left(\mathcal{I}_{a G\left(3_{1}\right)}\right)=<2 y-\left(2 x^{2}-1\right), 4 x^{2}+4 s x-3>.
$$

We have studied the representations corresponding to the points in this variety $V_{a}\left(\mathcal{I}_{a G\left(3_{1}\right)}\right)$, in [1].

Example 8 (The Figure Eight knot). Consider the group of the Figure Eight knot $4_{1}$.

Let

$$
G\left(4_{1}\right)=\pi_{1}\left(S^{3}-4_{1}\right)=\left|a, b: a b a^{-1} b^{-1} a=b a^{-1} b^{-1} a b\right|
$$

$$
\begin{aligned}
& \rho: G\left(4_{1}\right) \quad \longrightarrow \quad A(H) \\
& a \quad \rightarrow \quad \rho(a)=\left(s A^{-}, A\right) \\
& b \quad \rightarrow \quad \rho(b)=\left(s B^{-}+\left(A^{-} B^{-}\right)^{-}, B\right)
\end{aligned}
$$

be a representation of $G$ in the affine group of a quaternion algebra $H$. Assume that $\left\{A^{-}, B^{-},\left(A^{-} B^{-}\right)^{-}\right\}$is a basis of $H_{0}$.

Then, using the computer program Mathematica, we found that the parameters $x, y, s$ satisfy the equations:

$$
\begin{aligned}
p_{1}(x, y) & =1-6 x^{2}+4 x^{4}-2 y-4 y^{2}=0 \\
q_{1}(x, y, s) & =5+22 s x-9 x^{2}-16 s x^{3}+4 x^{4}+15 y-12 x^{2} y=0 \\
q_{2}(x, y, s) & =-5-10 s x+19 x^{2}-12 x^{4}-5 y-16 s x y+4 x^{2} y=0
\end{aligned}
$$

Therefore the variety of affine representation of $G\left(4_{1}\right)$ is

$$
V_{a}\left(\mathcal{I}_{a G\left(4_{1}\right)}\right)=<p_{1}(x, y), q_{1}(x, y, s), q_{2}(x, y, s)>.
$$

\section{REFERENCES}

[1] G. W. Brumfiel and H. M. Hilden. SL(2) representations of finitely presented groups, volume 187 of Contemporary Mathematics. American Mathematical Society, Providence, RI, 1995.

[2] V.Charette, T. Drumm, W. Goldman, and M. Morrill. Complete flat affine and Lorentzian manifolds. Geom. Dedicata, 97:187-198, 2003. Special volume dedicated to the memory of Hanna Miriam Sandler (1960-1999).

[3] D. Cooper and D. D. Long. Remarks on the A-polynomial of a knot. J. Knot Theory Ramifications, 5(5):609-628, 1996.

[4] R. H. Crowell and R. H. Fox. Introduction to knot theory. Based upon lectures given at Haverford College under the Philips Lecture Program. Ginn and Co., Boston, Mass., 1963.

[5] M. Culler and P. B. Shalen. Varieties of group representations and splittings of 3-manifolds. Ann. of Math. (2), 117(1):109-146, 1983.

[6] G. de Rham. Introduction aux polynômes d'un nœud. Enseignement Math. (2), 13:187-194 (1968), 1967 . 
[7] D. Fried and W. M. Goldman. Three-dimensional affine crystallographic groups. Adv. in Math., 47(1):1-49, 1983.

[8] W. M. Goldman. Nonstandard Lorentz space forms. J. Differential Geom., 21(2):301-308, 1985.

[9] F. González-Acuña and J. M. Montesinos-Amilibia. On the character variety of group representations in $\operatorname{SL}(2, \mathbf{C})$ and $\operatorname{PSL}(2, \mathbf{C})$. Math. Z., 214(4):627-652, 1993.

[10] H.M. Hilden, M. T. Lozano, and J. M. Montesinos-Amilibia. Peripheral polynomials of hyperbolic knots. Topology Appl., 150(1-3):267-288, 2005.

[11] H.M. Hilden, M. T. Lozano, and J. M. Montesinos-Amilibia. On the affine representations of the trefoil knot group. preprint, 2009.

[12] H.M. Hilden, M.T. Lozano, and J. M. Montesinos-Amilibia. Character varieties and peripheral polynomials of a class of knots. J. Knot Theory Ramifications, 12(8):1093-1130, 2003.

[13] H. M. Hilden, M. T. Lozano, and J. M. Montesinos-Amilibia. On the character variety of group representations of a 2-bridge link $p / 3$ into $\operatorname{PSL}(2, \mathbf{C})$. Bol. Soc. Mat. Mexicana (2), 37(1-2):241-262, 1992. Papers in honor of José Adem (Spanish).

[14] H. M. Hilden, M. T. Lozano, and J. M. Montesinos-Amilibia. On the arithmetic 2-bridge knots and link orbifolds and a new knot invariant. J. Knot Theory Ramifications, 4(1):81$114,1995$.

[15] T.Y.Lam. The algebraic theory of quadratic forms. Mathematics Lecture Note Series. W. A. Benjamin, Inc., Reading, Mass., 1973.

[16] R.Riley. Nonabelian representations of 2-bridge knot groups. Quart. J. Math. Oxford Ser. (2) 35, no. 138, 191-208, 1984. USA

(H.Hilden) Departament of Mathematics, University of Hawail, Honolulu, Hi 96822 ,

(M.T.Lozano) IUMA, Departamento de Matemáticas, Universidad de Zaragoza, Zaragoza 50009 , SPAIN

(J.M.Montesinos) Departamento de Geometría y Topología, Universidad Complutense, MADRID 28040, SPAIN 